\title{
Ultrafast Multichannel Optogenetic Stimulation of the Auditory Pathway for Optical Cochlear Implants
}

\author{
Dissertation \\ for the award of the degree \\ "Doctor rerum naturalium" \\ of the Georg-August-Universität Göttingen
}

within the doctoral program Sensory and Motor Neuroscience

of the Georg-August University School of Science (GAUSS)

\author{
submitted by \\ Daniel Keppeler
}

from Augsburg 


\section{Thesis Committee}

Prof. Dr. Tobias Moser

Institute for Auditory Neuroscience, University Medical Center Göttingen

Prof. Dr. Dr. Oliver Schlüter

Molecular Neurobiology, European Neuroscience Institute Göttingen

Department of Neuroscience, University of Pittsburgh

Dr. Katrin Willig

Nanoscale Microscopy and Molecular Physiology of the Brain, University Medical Center Göttingen

\section{Members of the Examination Board}

Prof. Dr. Thomas Dresbach

Anatomy and Embryology, University Medical Center Göttingen

Prof. Dr. Ralf Heinrich

Cellular Neurobiology, Schwann-Schleiden Research Center Göttingen

Camin Dean, PhD

Trans-synaptic Signaling, European Neuroscience Institute Göttingen 


\section{Contents}

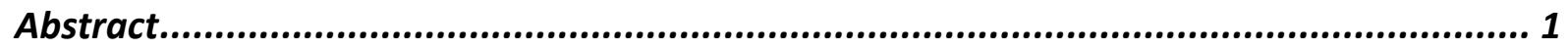

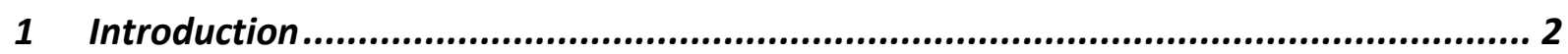

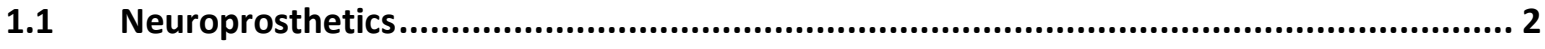

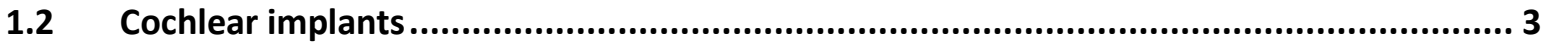

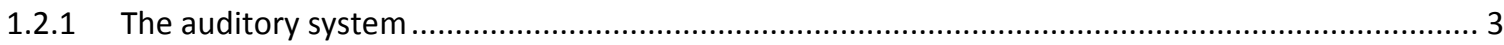

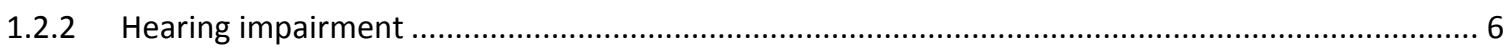

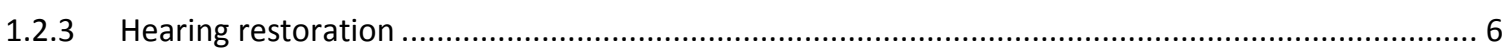

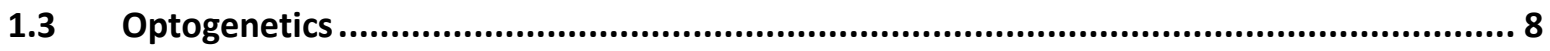



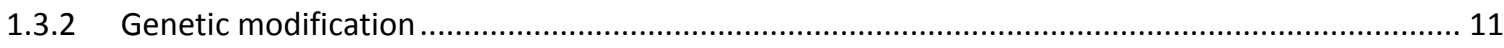

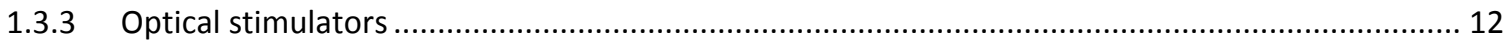

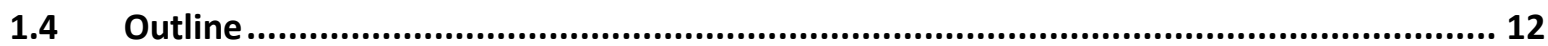

\section{Ultrafast optogenetic stimulation of the auditory pathway by targeting-optimized}

Chronos

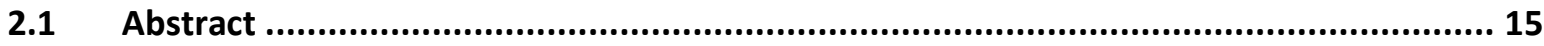



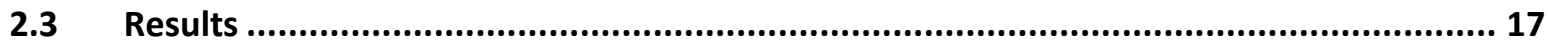

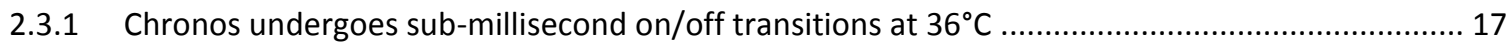

2.3.2 Improving the plasma membrane expression of Chronos ..................................................... 18

2.3.3 AAV-mediated expression of Chronos and Chronos-ES/TS in mouse SGNs .............................. 21

2.3.4 Chronos-mediated optogenetic neural stimulation of the auditory pathway: transuterine

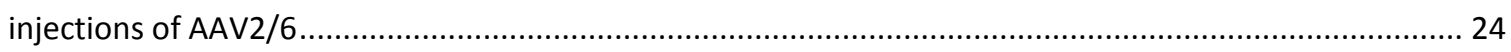

2.3.5 Chronos-ES/TS enables ultrafast optogenetic stimulation of the auditory pathway: oABRs......... 24

2.3.6 Chronos-ES/TS enables ultrafast optogenetic stimulation of the auditory pathway: recordings

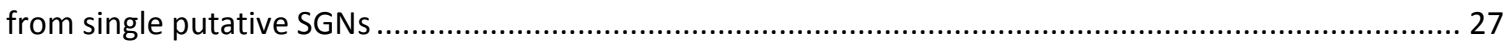

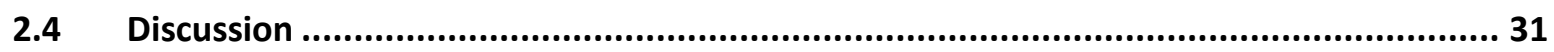

2.4.1 Biophysical characterization of Chronos and improving plasma membrane expression............... 31

2.4.2 Ultrafast Chronos-mediated stimulation of the auditory pathway .......................................... 33

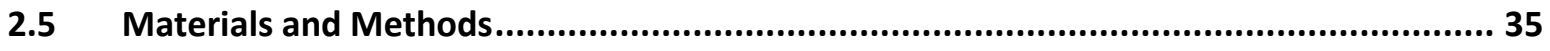

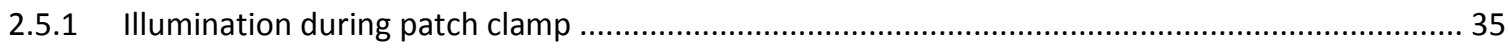

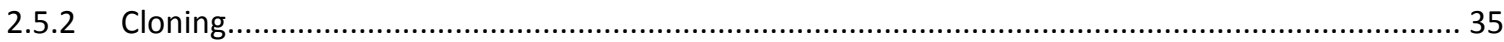

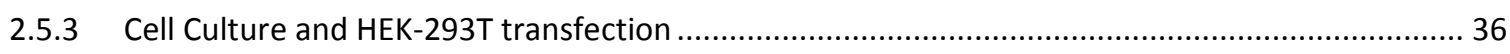

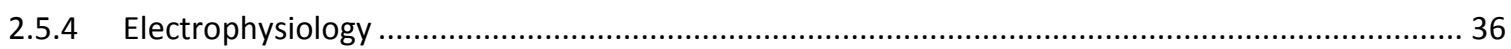


2.5.5 Dissociation and culture of hippocampal neurons, AAV infection and immunocytochemistry...... 37

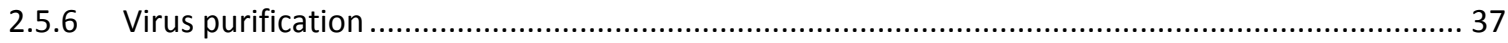

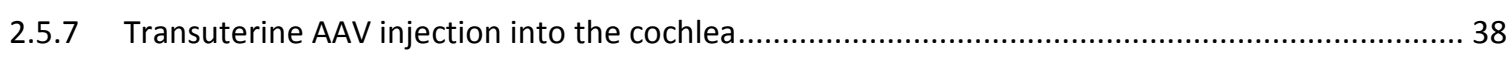

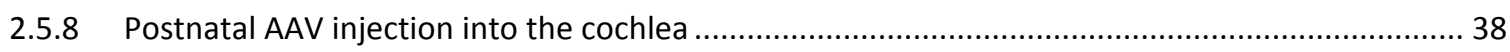

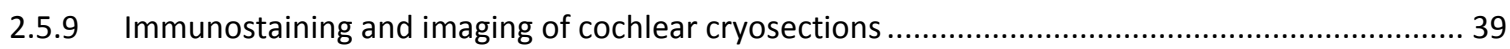

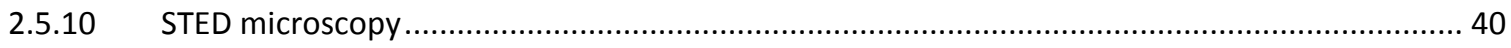

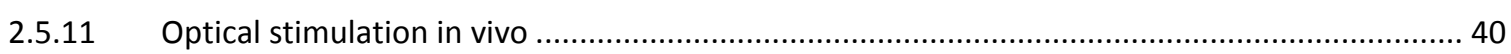

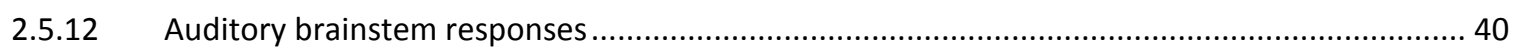

2.5.13 Juxtacellular recordings from single putative SGNs ......................................................... 40

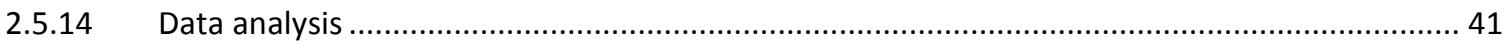

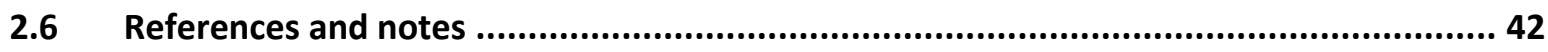

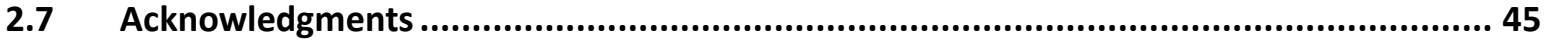

3 Multiscale, multimodal imaging of the native and implanted cochlea....................46

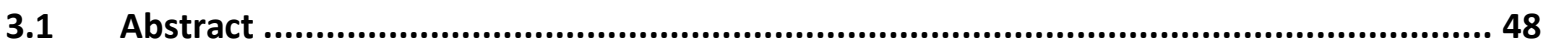

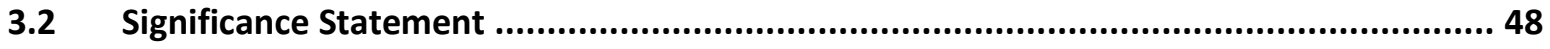

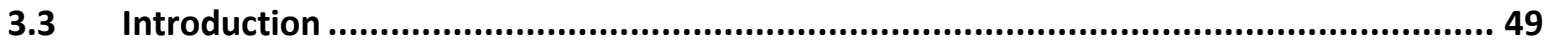

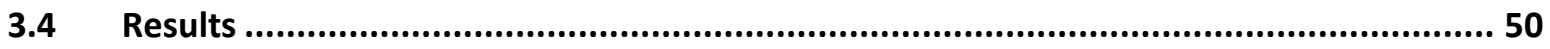

3.4.1 Multiscale, multimodal photonic imaging of cochlear morphology ......................................... 50

3.4.2 Quantifying scala tympani for cochlear implant design and insertion .......................................54

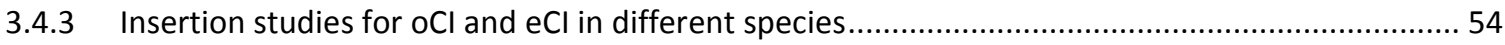

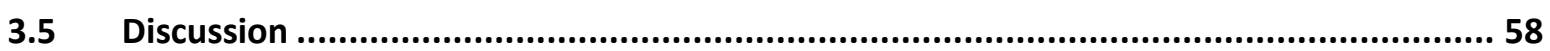

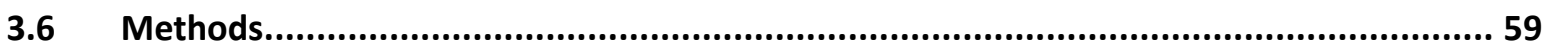

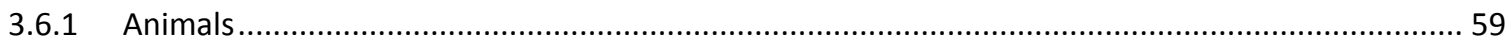

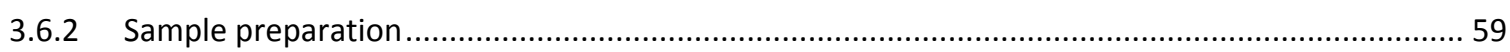

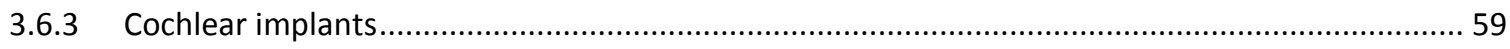

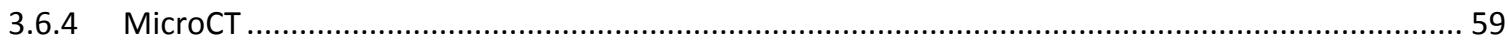

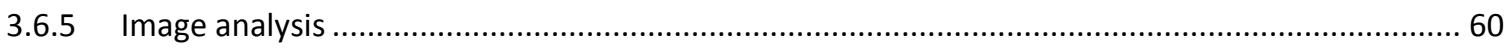

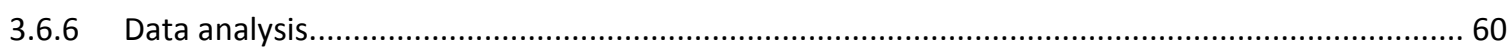

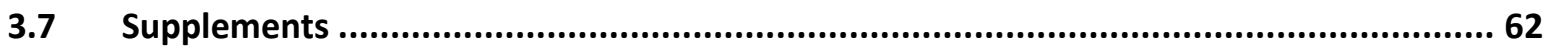

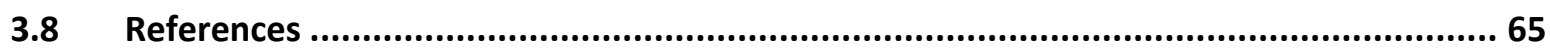

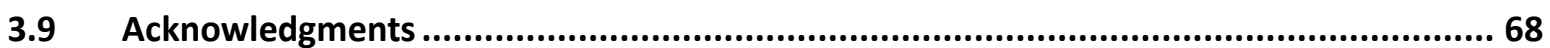

4 Towards optical cochlear implants: First multichannel optogenetic stimulation of the auditory pathway using microfabricated LED implants ....................................69

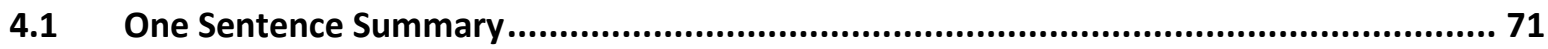

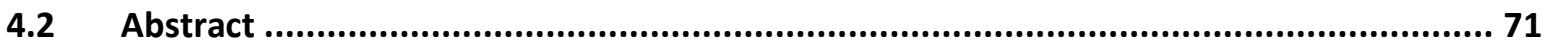




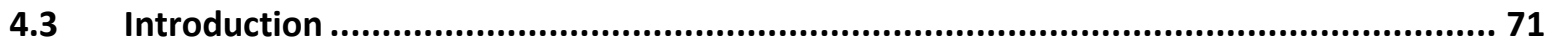

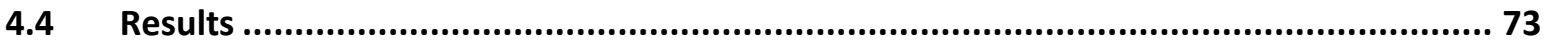

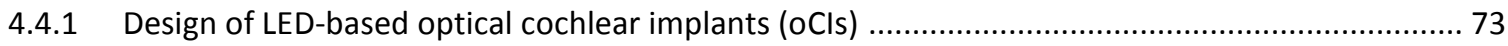

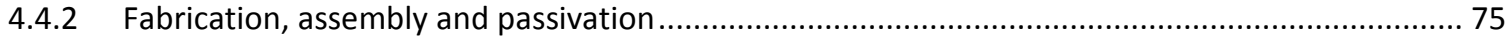

4.4.3 Electrical, optical and thermal characterization of the $\mathrm{oCl}$....................................................... 75

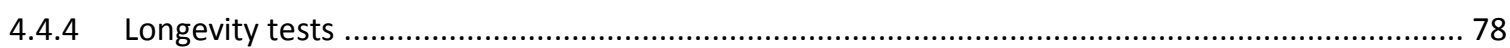

4.4.5 Optogenetic activation of the auditory pathway by multichannel oCls....................................... 78

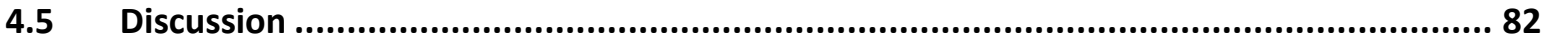

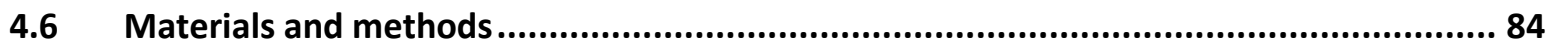

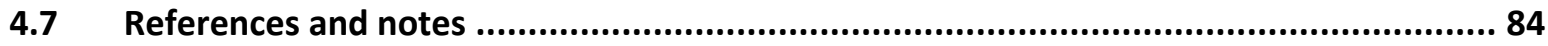

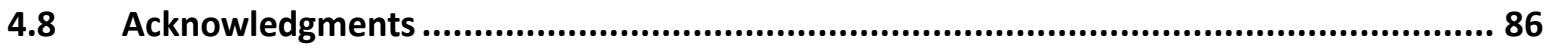

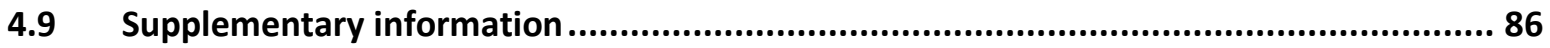

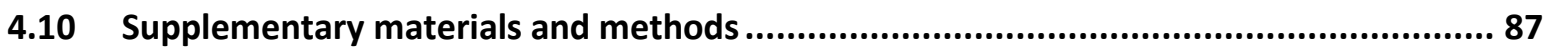

4.10.1 Microfabrication of the optical cochlear implant substrates ............................................... 87

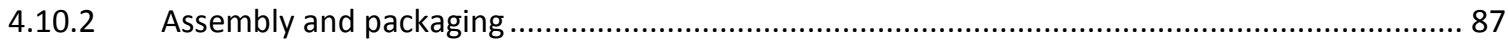

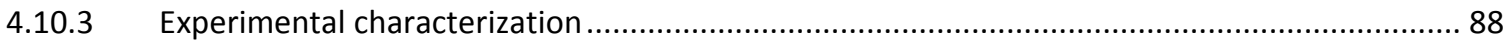

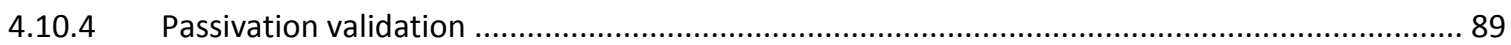

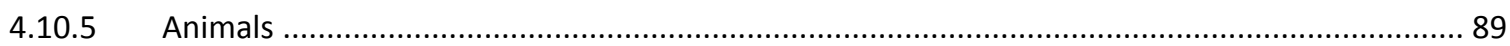

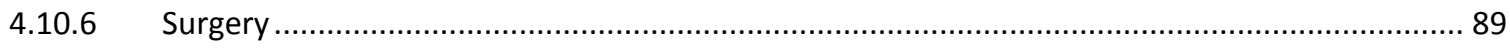

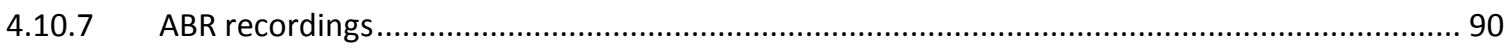

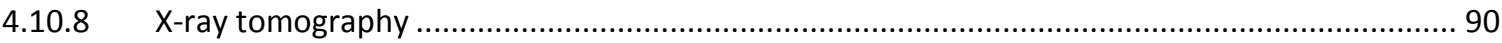

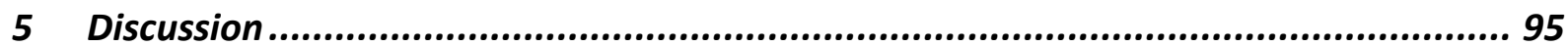

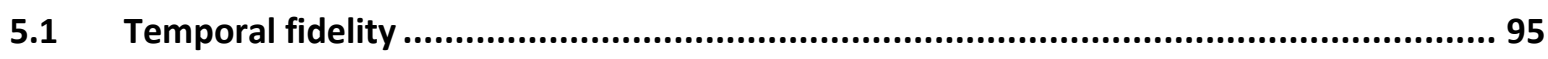

5.2 Optogenetic manipulation of spiral ganglion neurons ................................................ 96

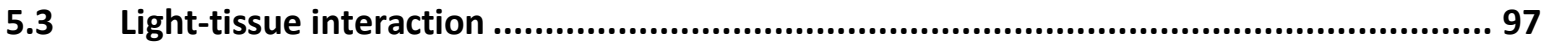

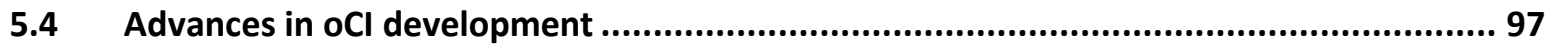

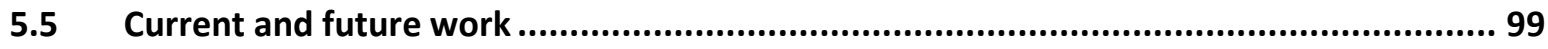

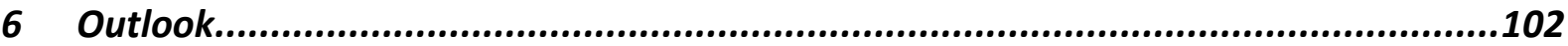

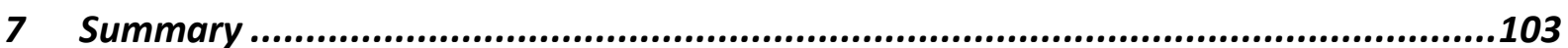

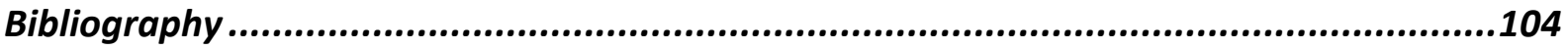

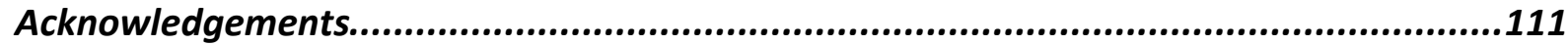

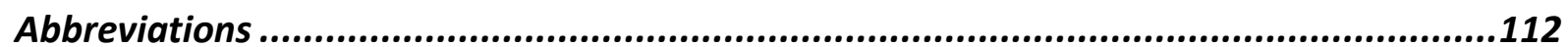



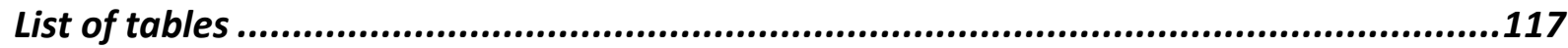

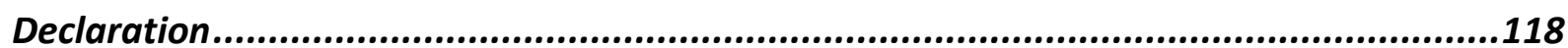


Curriculum vitae 


\section{Abstract}

In case of profound sensorineural hearing loss and deafness, cochlear implants (Cls) partially restore hearing and provide missing auditory information to the brain. Electrical stimulation of the spiral ganglion neurons (SGNs) enables speech understanding in the majority of the approximately 500,000 $\mathrm{Cl}$ users. However, the utility of current clinical $\mathrm{Cls}$ is limited by their wide current spread resulting in limited coding of spectral information. As light can be better confined in space, optical $\mathrm{Cls}$ (oCls) promise lower spread of excitation in the cochlea which might enable better speech comprehension in noisy background as well as music appreciation.

This thesis focuses on five key aspects for development of future Cls in research and clinical translation: (1) Characterizing a novel ultrafast optogenetic tool in the mouse cochlea: Chronos, a channelrhodopsin (ChR) supporting high temporal fidelity prerequisite for auditory coding. (2) Improving spiral ganglion neuron (SGN) expression levels and SGN targeting by the recently discovered adeno-associated virus (AAV)-PHP.B. (3) Enhancing ChR trafficking to the plasma membrane (Chronos-ES/TS). (4) Evaluating cochlear space for optical probes in common animal models employing phase-contrast X-ray tomography thereby providing a comprehensive library for morphological parameters relevant for $\mathrm{Cl}$ development in rodents and non-human primates. (5) Establishing first multichannel oCls based on microfabricated light-emitting diode (LED) arrays in channelrhodopsin-2 (ChR2)-expressing rats and their functional validation utilizing auditory brainstem responses (ABRs).

Taken together, the thesis demonstrates feasibility of optogenetic cochlea stimulation by expressing the ultrafast ChR Chronos and LED-based multichannel oCls. 
Introduction

\section{Introduction}

\subsection{Neuroprosthetics}

"We are the first generation able to decide what organs and senses we want to have." This slogan by the Cyborg Foundation raises hopes to enhance body functions beyond the natural capabilities of human bodies. Founder Neil Harbisson was born with an extreme form of colorblindness, which restricted him to perceive his environment in grayscale only. Since 2004 he has an implanted antenna which allows him to sense colors in the visible and invisible range of human eyes via audible vibrations inside his head. This permits him to not only extract colors used in daily live but also to tell the amount of ultraviolet (UV) radiation at the beach. The osseointegrated device implanted in Harbisson's head is a hybrid device of a bone-conducting hearing aid and a camera. Thus, the so-called cyborg antenna is not a neuroprosthesis by definition, but still illustrates the intention and purpose.

Neuroprosthetics offer the possibility to link medical auxiliaries to the human nervous system via neural interface to partially restore body functions. Often the field of neuroprosthetics is seen as a brain-computer interface only, but it offers far more: missing biological function can be replaced by medical devices to regain quality of life. Today, patients with diverse deficiencies in their senses can partially regain function. In the field of vision restoration, the retina implant has proven the most successful. The bionic eye electrically stimulates the visual nerve circumventing the degenerated photoreceptors, as for example in case of retinitis pigmentosa or geographic atrophy. Another prominent example for neuroprosthesis is the deep brain stimulator (brain pacemaker), which uses implanted electrodes in the central nervous system (CNS) to treat movement and neuropsychiatric disorders. The most successful clinical neuroprosthesis, however, is the cochlear implant $(\mathrm{Cl})$ which this thesis will focus on.

Despite all efforts in neuroprosthetics one should not underestimate the capabilities of the brain, which likely explains much of the success of e.g. the $\mathrm{Cl}$. Neuroplasticity, the ability of the CNS to adapt and rebuild synaptic connections in a stimulus-dependent manner, plays a major role in the great success observed in human rehabilitation. The reformation of neurons enables the brain to integrate new inputs and rebuilt a functional perception. Therefore, neural implants are most successful, the more peripheral the interface connects to the nervous system as this enables medical devices to make use of the neural processing of the brain along the many relay stations up to the cortex. 


\subsection{Cochlear implants}

\subsubsection{The auditory system}

The outer and middle ear: The adequate physical stimulus for the ear is sound. Pressure waves are collected by the pinna of the outer ear and directed onto eardrum that connects to the ossicular chain in the middle ear consisting of malleus, incus and stapes (Figure 1). The middle ear conveys the pressure waves to the fluid filled cochlea via the oval window. This mechanism is required for signal amplification because otherwise sound would be strongly attenuated due to the different inertia of gaseous and fluid media.
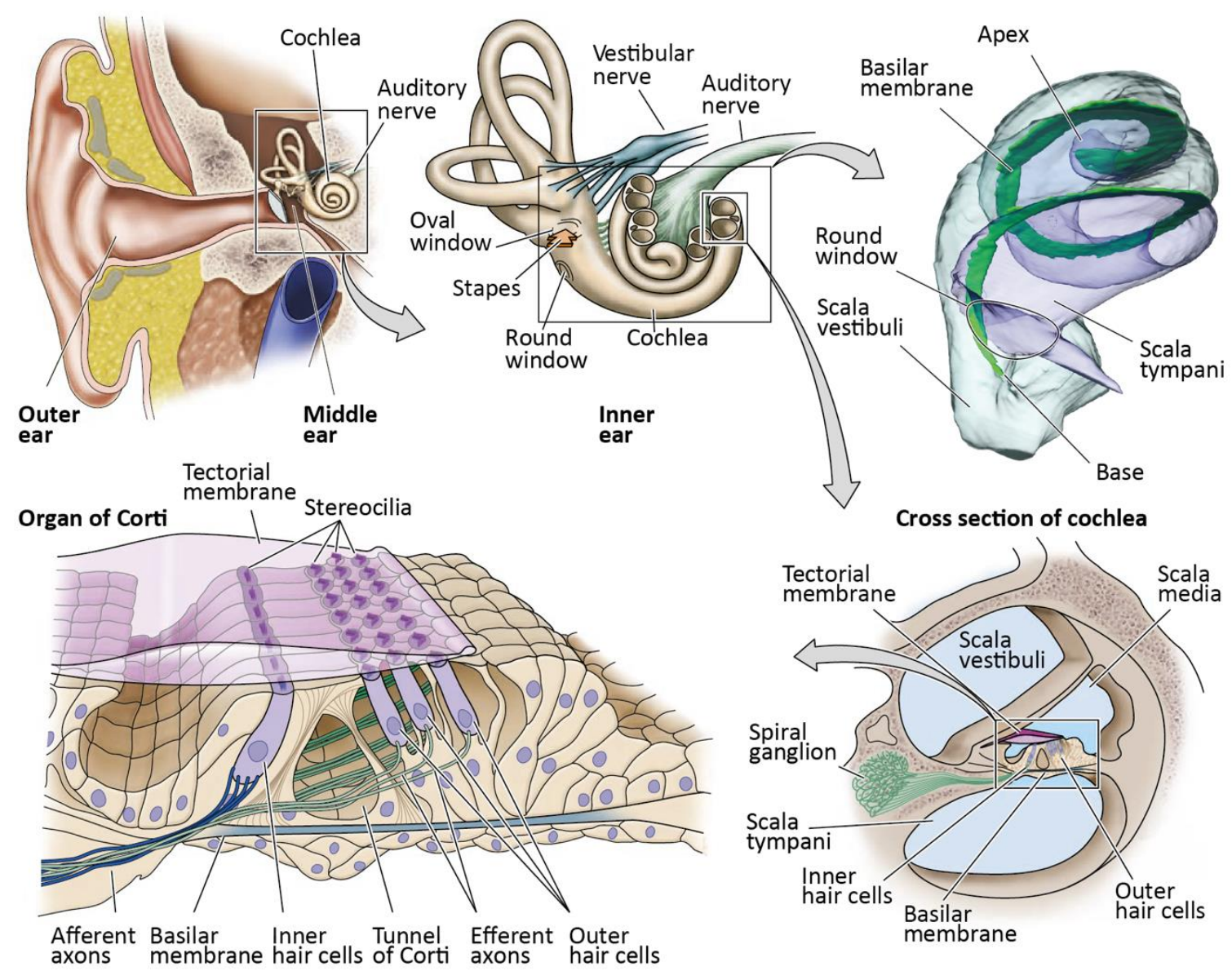

\section{Figure 1. The human ear}

Frontal section through the human ear starting at the outer ear (top left) to the inner ear (top center). The three scalae are depicted as a 3D mouse model (top right) as it is the focus of this thesis. Bottom shows cross-sections through a cochlear turn (right) and the organ of Corti (left). Modified and reprinted with permission from (Purves, 2018)

The inner ear: The cochlea itself, a bony, snail-shaped structure, consists of three distinct fluid-filled cavities (scala tympani, separated via the basilar membrane from the scala media, which in turn is separated via Reissner's membrane from the scala vestibuli) wrapped around the central pillar of the cochlea, the modiolus (Figure 1). At the cochlear apex, the scala tympani and the scala vestibuli are 
connected via the helicotrema, and both are filled with a liquid called perilymph. At the cochlear base, two membrane-covered windows are found: the oval window (where the footplate of the stapes ends) in the scala vestibuli and the round window in the scala tympani. When the footplate of the stapes moves, it also moves the oval window membrane. Perilymph in the scala vestibuli is pushed towards the cochlear apex and in turn pushes the perilymph in the scala tympani via the helicotrema towards the round window, which is bulged outwards.

Since not all intracochlear structures are rigid, pressure waves cause motion while traveling through the cochlea - most importantly at the basilar membrane. Due to its physical properties - i.e. gradual decrease in width and increase in stiffness from the cochlear apex towards the base - its displacement depends on the frequency of pressure waves: While high frequencies deflect the stiff and narrow part at the cochlear base, low frequency waves travel up to the cochlear apex and mainly deflect the wider and more flexible part of the basilar membrane (Figure 2). As a result, a frequency-place code in the cochlea is established, where different frequencies of pressure waves cause basilar membrane deflections at different locations in the cochlea.

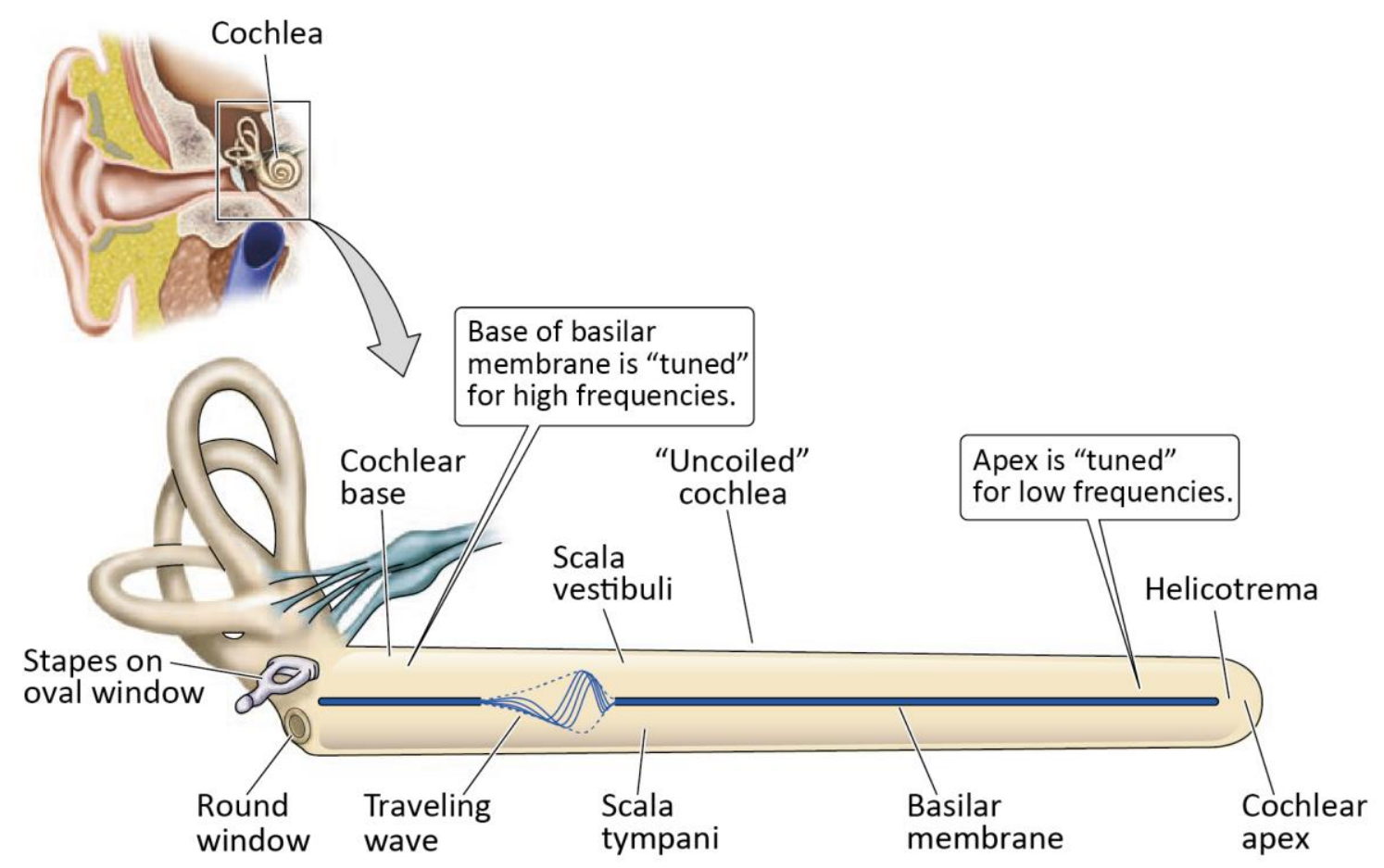

Figure 2. Travelling wave along the uncoiled cochlea

The uncoiled cochlea with the basilar membrane showing a travelling wave at a given instance. Modified and reprinted with permission from (Purves, 2018).

Mechanotransduction: The responsible organ for signal transduction - the organ of Corti - is located on the basilar membrane in the scala media. It is covered by a second membrane, the tectorial membrane, which is connected to the basilar membrane via the rods of Corti (also known as pillar cells) and surrounded by endolymph. The sensory cells in the organ of Corti, the so-called hair cells, 
detect cochlear vibrations via their stereociliar bundles. While the outer hair cells (OHCs) serve as mechanical amplifier, the inner hair cells (IHCS) are the "genuine" sensory cells conveying the sound information to type I spiral ganglion neurons (SGNs) at their afferent synapses. The stereocilia of are connected via tip-links. Mechanically activated cation channels in the membrane of stereocilia are partially open in their resting state allowing moderate potassium influx from the endolymph into the hair cells generating a rather depolarized resting potential. A propagating wave along the cochlea deflects the basilar membrane thus the organ of Corti in respect of the tectorial membrane. This leads to deflection of the hair cell bundles. Depending on the direction of movement this can either lead to an increase or decrease in tension on the cation channel i.e. a more open or closed state, respectively. Opening of mechanotransducer channels causes additional potassium influx further depolarizing the cell: receptor potential. In IHCS the receptor potential activates voltage-gated calcium channels IHCS causing to calcium influx at the presynaptic ribbon-type active zones. The incoming calcium triggers the release of the neurotransmitter glutamate into the synaptic cleft. The released glutamate subsequently activates afferent fibers of spiral ganglion neurons (SGNs), the primary neurons in the auditory system, which - if activation is strong enough - initiate action potentials. This process converts the mechanical sound signal into an electrical nerve signal. The somata of the bipolar SGNs are located in Rosenthal's canal within the modiolus, with their peripheral neurites reach to the base of IHCs. Their central neurites form the auditory nerve and project to the cochlear nucleus of the brainstem, thus sending information about acoustic signals from the ear to the central nervous system.

Neural encoding of sound: Basically, sound waves are characterized by their frequency (number of cycles per time window) and amplitude (sound pressure or intensity). While frequency is perceived as pitch, amplitude corresponds to loudness. As mentioned above, the travelling wave deflects the basilar membrane at different locations resulting in a distinct IHC activation pattern. The subsequent activation of neuronal populations by sound waves of different frequencies is maintained throughout the auditory pathway up to primary auditory cortex - the so-called tonotopy. Thereby, the brain is able to infer the frequency of an acoustic signal by the spatial activation pattern of neurons: pitch perception. Loudness, on the other hand is coded by two mechanisms: a larger amplitude leads to a greater vibration of the basilar membrane leading to (1) a stronger deflection of the stereocilia, leading to a greater receptor potential and ultimately results in a higher rate of action potential firing in the auditory nerve fibers and (2) a broader stimulation of IHCs, thus activating a larger population, thus more activated SGNs.

This is a simplified view of hearing from the outer ear up to the first stage of the CNS. From there processing continues along many parallel pathways in relay stations including the superior olivary complex, nuclei of the lateral lemniscus, inferior colliculus and medial geniculate body up to the 
Introduction

primary auditory cortex. The central auditory pathway achieves not only pitch and loudness perception, but also e.g. sound localization through binaural integration. Finally, the information is integrated with other cortical areas in order to produce behaviorally relevant output to auditory signals.

\subsubsection{Hearing impairment}

According to the World Health Organization, approximately 466 million people worldwide - which account for $6.1 \%$ of the human population - suffer from disabling hearing loss, resulting in an economic impact of about $\$ 750$ billion globally spent on the treatment of hearing loss (WHO, 2018). As of today, 1 out of 800 children is born with profound hearing impairment, $15 \%$ of the world's adult population experience at least mild hearing loss and approximately one third of the population above 65 years of age are affected by disabling hearing loss. While approximately half of the cases of hearing impairment in children can be assigned to genetic mutations, the remaining ones are caused by environmental factors. In adults, acquired hearing loss - mainly noise-induced or age-related contribute to make hearing loss the most prevalent sensory disorder. Consequences of hearing loss are severe and diverse: During early childhood, and especially during critical windows of development, the auditory system relies on sensory input in order to establish, mature and maintain the sense of audition. Furthermore, the acquisition of vocal speech fully depends on the auditory system. During adulthood, hearing impairments can cause decreased professional capabilities, risk for diseases such as depression, and decreased quality of life in general. In elderly, hearing impairment has been linked to cognitive decline and dementia. Furthermore, over all ranges of age, hearing impairment causes social isolation which in turn is linked to a diversity of secondary disadvantages.

\subsubsection{Hearing restoration}

When hearing fails, rehabilitation depends on the nature and degree of the respective hearing impairment. For diagnosis subjective audiometry like determination of hearing thresholds and speech recognition as well as objective audiometry like otoacoustic emissions or auditory brainstem responses (ABRs) are used to classify the hearing impairment in severity and etiology. Depending on the extend of hearing loss, ranging from mild (20-40 dB) to moderate (40-70 dB) or severe (70-90 dB) form, patients might benefit from hearing aids, middle ear implants or bone conduction implants. When these treatments fail, in case of profound sensory neural hearing loss (SNHL, 90-120 dB) or in the deaf a cochlear implant $(\mathrm{Cl})$ is currently the best option to partially restore hearing. This requires the auditory nerve to not be severely compromised or missing. In this rare case, implants need to stimulate structures more central to the auditory nerve, reducing hearing restoration outcome compared to $\mathrm{Cls}$ (Peng et al., 2018). Most prominent example is the auditory brainstem implant (ABI), which can restore 
Introduction

a hearing percept, but speech perception is limited and the craniotomy is not without significant risk (Schwartz and Wilkinson, 2017).

Electrical cochlear implants $(\mathrm{eCl})$ consist of an internal part with an intracochlear electrode array encapsulated in silicone linked to an extracochlear stimulator. The stimulator is built of two parts: An implanted receiver connected to the electrodes in the cochlea and a speech processor including battery supply, microphones and a transmitter magnetically fixed on the skin. While the microphone picks up sounds from the environment, the acoustic signal is decomposed into frequency bands and transcutaneously transmitted to the receiver/stimulator delivering current pulses to the electrode contacts within the cochlea, directly stimulating the SGNs, thereby circumventing the non-functional sensory organ of Corti. Current flows from an active single electrode contact either to a reference electrode located in the temporalis muscle or in the housing of the receiver/stimulator in the so-called monopolar stimulation; or to a neighboring electrode, in the so-called bipolar stimulation. Thereby, the electrode array - located in the scala tympani - makes use of the tonotopic axis of the cochlea: a electrode contact located at the base primarily excites SGNs coding for high frequencies, while an electrode contact placed in the apex stimulates neurons coding for lower frequencies. Most implant systems make use of the monopolar configuration, as performance is at least comparable to bipolar stimulation (Zhu et al., 2012; Zwolan et al., 1996), but require far less battery power for auditory percepts (Pfingst and Xu, 2004) compared to the bipolar modality.

Current $\mathrm{Cls}$ enable patients to comprehend open speech in quietness and to some degree in an environment with background noise, but the appreciation of music is limited (Kohlberg et al., 2014). Decisive for good speech comprehension is the amount of stimulation channels - in case of an $\mathrm{eCl}$ the number of electrodes. As reported by Shannon et al. (1995) speech recognition in quietness only requires 4 stimulation channels, whereas in noise 8 or more channels are necessary (Smith et al., 2002). For melody recognition Shannon et al. (Shannon et al., 2004) suggests to at least provide 48 spectral channels. While commercial Cls currently provide 12-24 physical channels, only a low number of effective channels can be used (Friesen et al., 2001) due to wide current spread within the highly conductive perilymph of scala tympani (Kral et al., 1998). This leads to broad activation of large subpopulations of SGNs resulting in reduced frequency resolution. If it comes to speech processing and coding strategy, the independent sites are limited to 4-8 (Fishman et al., 1997; Friesen et al., 2001; Garnham et al., 2002; Kiefer et al., 2000; Wilson, 1997).

Another obstacle for $\mathrm{Cls}$ is the placement within the scala tympani: As the implant is commonly located close to the lateral wall of scala tympani, the large distance between stimulation site and SGNs in the Rosenthal's canal further decreases performance for eCls (Cohen et al., 2006). Furthermore, Cls barely cover the whole length of scala tympani (Lee et al., 2010; Wilson, 2008) due to its coiled structure and decrease in diameter (chapter 3). 
Optical stimulation promises to bypass the bottleneck of current eCls: Light can be spatially confined and does not lead to channel cross-talk (Figure 3, also see reviews (Dombrowski et al., 2018; Jeschke and Moser, 2015; Richter and Tan, 2014)). However, optogenetic stimulation requires genetic modification of the auditory nerve to render the cells sensitive for light stimulation.

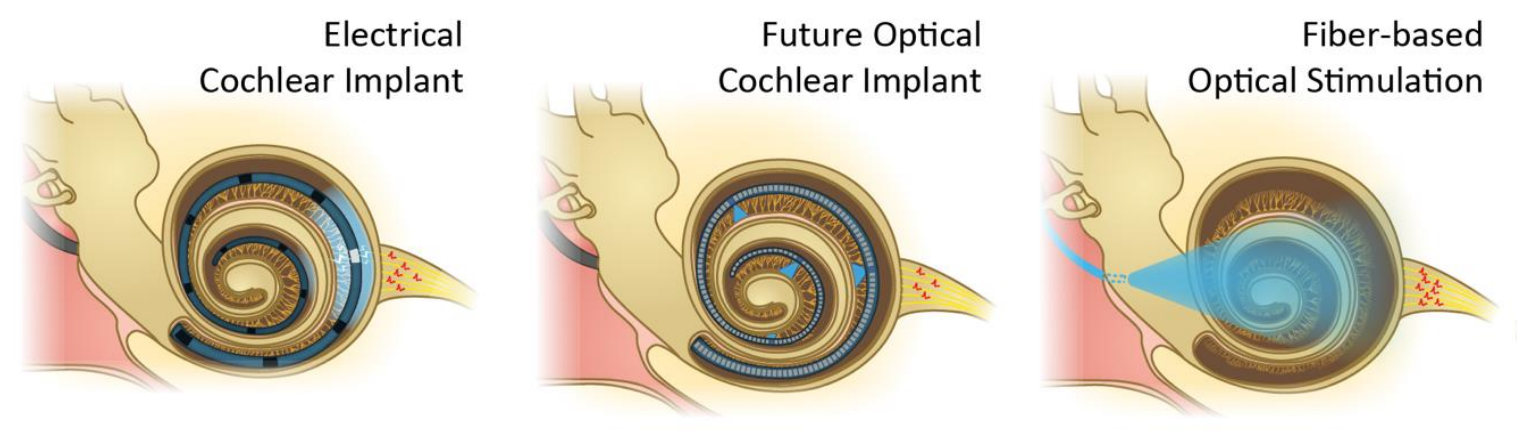

Figure 3. Schematic overview of different cochlear stimulation modalities

Electrical cochlear implant (left) typically use 12-24 stimulation channels while for optical cochlear implants (middle) an increase of stimulation channels by an order of magnitude might be possible. The right panel shows fiber-based optical stimulation used for in vivo characterization in animal models. Modified and reprinted with permission from (Moser, 2015).

\subsection{Optogenetics}

Optogenetics enables selective control of target cells with light stimuli in space and time. The use of light-sensitive proteins to control cellular function is considered as one of the most revolutionary innovations in the life sciences in recent years. Channelrhodopsins (ChR) were discovered around the millennium in the green alga Chlamydomonas reinhardtii as a light-sensitive membrane protein used in phototaxy. The DNA sequence was identified in the Hegemann group as encoding for large microbialtype rhodopsins. In collaboration with Nagel and Bamberg, expression and subsequent analysis in Xenopus oocytes of the two types ChR1 and ChR2 was achieved (Nagel et al., 2002, 2003). Several groups started working on these newly described blue light-gated ion-channels offering the possibility to manipulate cells electrically. In 2005, ChR2 was used to drive neural activity with a millisecond precision in hippocampal neuron cultures (Boyden et al., 2005). Shortly after, first in vivo studies demonstrated neural photostimulation with ChR2 in freely moving mice (Adamantidis et al., 2007) using a lentiviral gene transfer. Depending on the choice of opsin, discussed in the next chapter, it is not only possible to excite, but also inhibit neural activity (Figure 4).

This was the beginning of a new era. The two main features of optogenetic applications to neuroscience are (1) matching the dynamics of neuronal action potentials and synaptic currents and (2) spatial selectivity within the tissue excluding stimulation of neighboring areas. 
Electrical stimulation

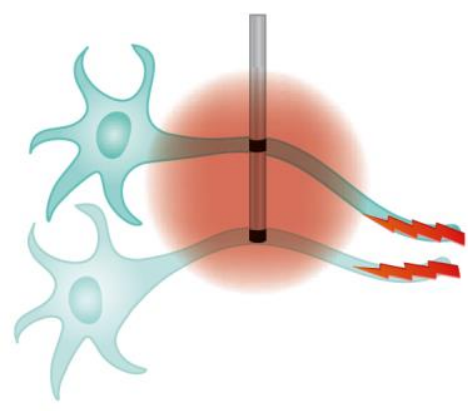

Optogenetic excitation

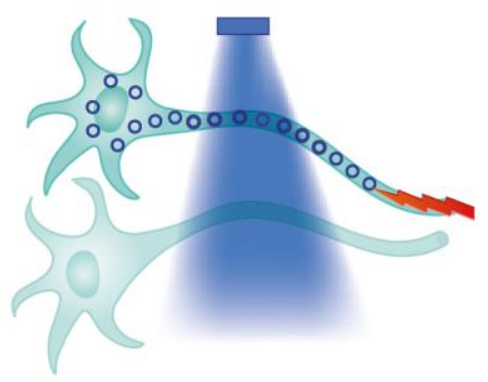

Optogenetic inhibition

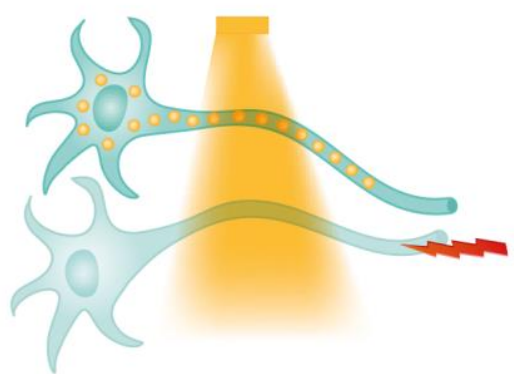

Figure 4. Principle of optogenetics in neuroscience

Illustration of the stimulation principles of electrical and optogenetic stimulation modalities. Optogenetic stimulation allows cellular and projection specificity, which is not feasible for electrical stimulation. The example shows electrical stimulation (left) vs. blue channelrhodopsin excitation (middle) vs. yellow halorhodopsin inhibition (right). Reprinted with permission from (Deisseroth, 2011).

\subsubsection{Opsins}

The microbial optogenetic tools belong to the type I opsins and are found in all three domains of life: Archaea, bacteria and eukaryota. Type II opsins (animal type) are only found in higher eukaryotes responsible for vision or the circadian rhythm (Shichida and Yamashita, 2003). Both superfamilies belong to the seven-transmembrane proteins (Yizhar et al., 2011a). Here, we focus on the type I opsin family that are the main tools in optogenetics. Next to the light-sensitive ion channels, the bacteriorhodopsins (BR) and halorhodopsins (HR) compose the group of light-gated ion pumps (Figure 5). While ChRs are selective for cations and BRs are outward proton pumps, HRs are light-activated chloride pumps, thus, - in neurons - the first two lead to excitation, while the latter lead to inactivation of the target cell (Figure 4). Recently, a light-driven inward proton pump was described (Inoue et al., 2016) which enables further optogenetic control of cells. This thesis focuses on ChRs as it is most relevant for optogenetics in SGNs.

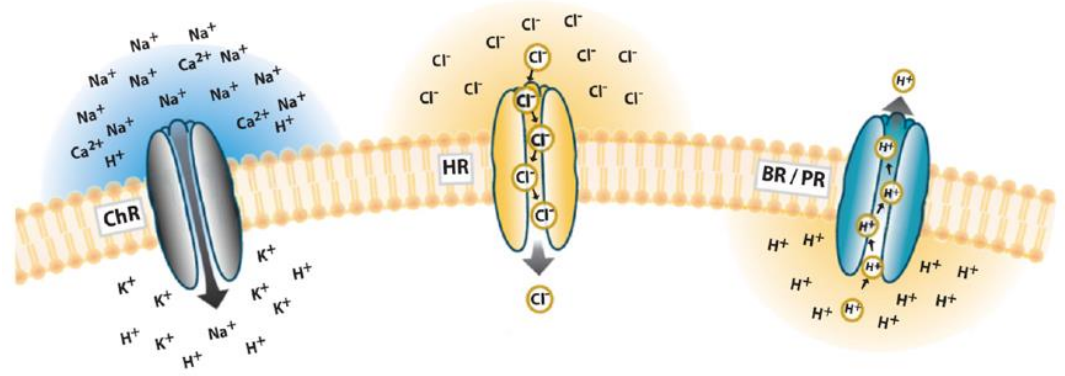

\section{Figure 5. Schematic of basic function of rhodopsins}

Light-gated cation channel channelrhodopsin (ChR, blue), chlorid pump halorhodopsin (HR, yellow) and outward proton pump bacteriorhodopsin (BR, turquoise). Modified and reprinted with permission from (Yizhar et al., 2011b) 
For the light-gated ion channel to function it requires a retinal molecule which is a vitamin A-related organic cofactor assisting as the photon detector. In conjunction with the protein complex the retinal is termed rhodopsin. In channelrhodopsin, the most prominent optogenetic tool, the light-dependent ion channel undergoes several molecular transitions, which are described by several state maps based on spectroscopic methods (Bamann et al., 2008; Ernst et al., 2008; Neumann-Verhoefen et al., 2013; Ritter et al., 2008). Upon light stimulation, the molecule is thought to cycle through these states. In brief, the retinal absorbs photons leading to a series of conformational changes. The photoisomerization happens within nanoseconds, leading to an opening of the internal gate within sub-millisecond scale $\left(\tau_{\text {on }}\right)$, thus, enabling cations to pass through the pore (Kuhne et al., 2015). After light stimulation, the channel remains open - completing the photocycle - the so-called off-kinetics ( $\left.\tau_{\text {off }}\right)$. The time interval after light-off varies greatly among ChRs. ChR2 - best activated at a wavelength of around $460 \mathrm{~nm}$ (Nagel et al., 2003) - has a $\tau_{\text {off }}$ of about $10 \mathrm{~ms}$ (Gunaydin et al., 2010) which limits the temporal fidelity for high stimulation rates. Mutations in pore residues shortens the $\tau_{\text {off }}$ as in E123T (ChETA variant) to about $4 \mathrm{~ms}$ allowing sustained spiking of up to $200 \mathrm{~Hz}$ (Gunaydin et al., 2010). However, the increase in temporal fidelity comes at the cost of a decrease in light sensitivity since fewer channels remain in the open state on the photocurrent (Yizhar et al., 2011b). Another promising mutation, despite the slow $\tau_{\text {off }}$ of $16 \mathrm{~ms}$, is the calcium translocating ChR (CatCh). CatCh supports rapid repolarization due to enhanced calcium influx speeding up hyperpolarization and has an increased light sensitivity of about 70-fold (Kleinlogel et al., 2011). Chronos, a recently described channelrhodopsin isolated from Stigeoclonium helveticum (Klapoetke et al., 2014) promises to unite high light sensitivity and fast kinetics which will be discussed in chapter 2 .

Also, ChRs with different spectral properties are available: The first discovered ChRs best activated at a wavelength of around $530 \mathrm{~nm}$ is Volvox carteri ChR1 (VChR1) (Zhang et al., 2008). Soon modified variants like ReaChR and C1V1 were designed (Lin et al., 2013; Prigge et al., 2012; Yizhar et al., 2011c). More recently, the most red-shifted ChR to date - namely Chrimson - was identified in Chlamydomonas noctigama with an absorption peak at $590 \mathrm{~nm}$ (Klapoetke et al., 2014). Following Chrimson, two faster variants were engineered in the Bamberg lab (Mager et al., 2018): f-Chrimson and vf-Chrimson show channel kinetics close to Chronos. Red-shifted optogenetics offers advantages given the longer stimulation wavelength: higher transmission in organic tissue, thus allowing deeper tissue penetration and no phototoxic side-effects described for blue light stimulation. An overview of the increasing range of ChR variants and mutations that are generated and discovered is depicted in Figure 6. 


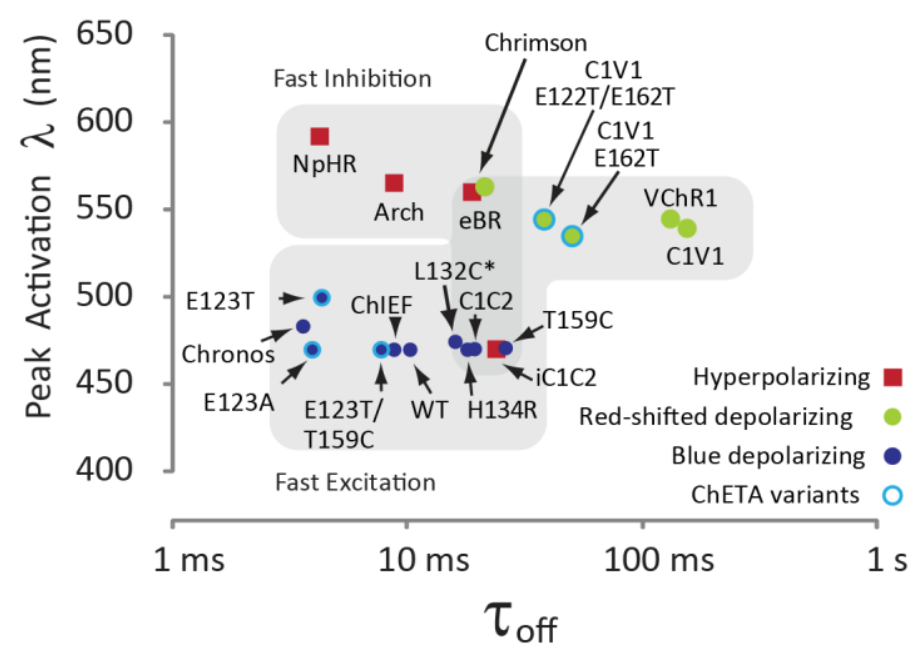

Figure 6. Opsin kinetics and peak absorption wavelength for common optogenetic tool variants

Data were recorded in neurons at room temperature except for ${ }^{*}$ CatCh which was tested in HEK cells. Modified from (Jeschke and Moser, 2015; Yizhar et al., 2011b).

\subsubsection{Genetic modification}

Neurons need to be rendered sensitive to light by expression of opsins. This is achieved via genetic modification of the target neurons - the spiral ganglion neurons for the sake of this thesis. For basic research, transgenic lines are readily available for mice (Zeng and Madisen, 2012) and rats (Witten et al., 2011). For experiments in chapter 4, we used ChR2-expressing rats, in which ChR2 expression is driven by the broad neuronal promoter Thy1.2. However, for higher flexibility regarding the use of alternative opsins and later clinical translation to humans, the need for alternative genetic modification arises.

Here the most promising approach considered today is gene transfer via viral vectors. This method allows easy and fast incorporation of newly designed components. Further, tissue and cell specificity can be regulated by the choice of promoter, capsid of specific viral subtypes, and application site. Adeno-associated viruses (AAVs) seem to be the best candidate to date as researchers for vision restoration were successfully running clinic trials to replace the gene RPE65 (reviewed in (Dalkara et al., 2016)).

AAVs provide a number of advantages: (1) AAVs are not linked to any known cause of disease to date, (2) show low immune responses and (3) are replication-deficient. AAVs are able to integrate into the host genome at a safe harbor, however, common modifications on the AAV backbone restrict integration to form episomal DNA structures allowing long-term expression in post-mitotic cells. AAVs can effectively target different cell types across the cochlea (Shu et al., 2016) and were successfully used to deliver ChRs to the SGNs (Duarte et al., 2018; Hernandez et al., 2014; Mager et al., 2018). 
Introduction

\subsubsection{Optical stimulators}

To be able to stimulate SGNs with light, an appropriate emitter needs to be placed in the coiled cochlea. The choice of emitter needs to be deliberately weighed against pros and cons of the respective optoelectronic device. This topic is covered in chapter 3 and 4 . An overview of available optical stimulators is provided here.

In principle, the emitter needs to be power-efficient, small enough to fit in the cochlea providing more stimulation channels than currently used for eCls and bright enough to drive optically evoked action potentials in the light sensitive neurons. An oCl can be designed either as an active stimulator generating light at the emitter site - or as an passive stimulator with waveguides transporting photons from an external light source to the target site of excitation.

Light-emitting diodes (LEDs) promise to be an ideal candidate for an active oCl: Wafer-level processed LEDs based on gallium nitride (GaN) achieve power efficiencies of more than 50 \% (Laubsch et al., 2010) and can be miniaturized to fit more than 100 emitters within the size of a mouse cochlea (Goßler et al., 2014). Next to conventional LEDs, organic LEDs (OLEDs) might provide an alternative promising high spatial resolution and mechanical flexibility at dimensions below cellular size (Steude et al., 2016). Passive oCls, however, offer several advantages and have been similarly miniaturized to accommodate to the cochlear space (Schwaerzle et al., 2016a): The light generating element is located outside the spatially confined cochlea thus providing additional space. Further, potential heat is not presented to the sensitive inner ear. On the downside, coupling of light into waveguides comes with transmission loss (Cho et al., 2010; Zorzos et al., 2010).

\subsection{Outline}

This thesis aims at providing basic insight for optogenetic cochlear stimulation by addressing the following points also relevant for future translation:

- Characterization of the most promising blue-light sensitive channelrhodopsins in the auditory system to estimate its potential for optogenetic research and hearing restoration

- Evaluation of cochlear space across animal models commonly used in auditory neuroscience

- Assessment of first multi-channel optical cochlear implant stimulation in the rat cochlea 


\section{Ultrafast optogenetic stimulation of the auditory pathway by targeting-optimized Chronos}

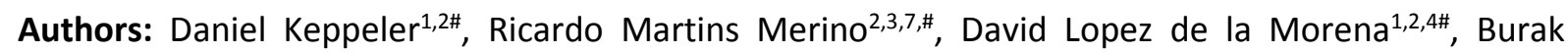
Bali $^{1,2,5,0}$, Antoine Tarqiun Huet ${ }^{1,3,4,0}$, Anna Gehrt ${ }^{1,6}$, Christian Wrobel ${ }^{1,6}$, Swati Subramanian ${ }^{1,2}$, Tobias Dombrowski $^{1,5,8}$, Fred Wolf ${ }^{6,7,8}$, Vladan Rankovic ${ }^{1,5^{*}}$, Andreas Neef ${ }^{2,3 *}$, Tobias Moser ${ }^{1,2,3,4,6,8^{*}}$

\section{Affiliations:}

${ }^{1}$ Institute for Auditory Neuroscience and InnerEarLab, University Medical Center Göttingen, Germany.

${ }^{2}$ Göttingen Graduate School for Neurosciences and Molecular Biosciences, University of Göttingen, Germany.

${ }^{3}$ Biophysics of neural computation group, Bernstein Center for Computational Neuroscience Göttingen, Germany.

${ }^{4}$ Auditory Neuroscience and Optogenetics laboratory, German Primate Center, Göttingen, Germany.

${ }^{5}$ Restorative Cochlear Genomics group, ${ }^{4}$ Auditory Neuroscience and Optogenetics laboratory, German Primate Center, Göttingen, Germany.

${ }^{6}$ Collaborative Research Center 889, University of Göttingen, Göttingen, Germany.

${ }^{7}$ Bernstein Center for Computational Neuroscience Göttingen, Germany.

${ }^{8}$ Neurophysics group, Max Planck Institute for Dynamics and Self-Organization, Göttingen, Germany.

${ }^{9}$ Center for Nanoscale Microscopy and Molecular Physiology of the Brain, Göttingen, Germany.

$\#{ }^{\circ}$ equal contribution

${ }^{\S}$ Department of Otorhinolaryngology, Head and Neck Surgery, Ruhr University Bochum, St. Elisabeth Hospital, Bochum, Germany.

*To whom correspondence should be addressed:

Dr. Vladan Rankovic, Email: vrankovic@dpz.eu

Dr. Andreas Neef, Email: andreas@nld.ds.mpg.de

Dr. Tobias Moser, Email: tmoser@gwdg.de

Copyright notice:

This material is reprinted by permission from Wiley in accordance to their copyright and licensing regulations. (C) owned by the authors, 2018. 


\section{Author contributions:}

D.K., V.R., F.W., A.N. and T.M. designed the study. D.K. performed o/aABR recordings, immunohistochemistry and FP expression analysis. A.G., T.D. and C.W. performed initial o/aABR recordings. R.M. performed patch-clamp recordings, B.B. performed immunohistochemistry. S.S. and D.L.M. performed initial cloning. V.R. performed AAV injections, HEK293T and hippocampal neuron experiments, confocal and STED microscopy, cloning and production of the viruses. D.L.M. performed recordings from single SGNs and OABRs. A.H. developed analysis toolboxes for ABRs and recordings from single SGNs and performed analysis of electrophysiological data. All authors analyzed data and contributed to the writing of the manuscript. T.M., D.K., V.R. and A.N. took a lead on preparing the manuscript.

Detailed author contributions of D. Keppeler (D.K.):

- Experimental work

- ABR recordings (A.G., T.D., C.W. contributed initially to data for Figure EV1)

- Establishing and initializing immunohistochemistry and imaging

- Confocal (Figure 4B and initially for Figure 4C-D, continued by B.B.)

- STED (together with V.R.)

$\circ$ Excluding Figure 1, Figure 2B, Figure 3A, Figure 4C-D, Figure 6, Figure EV2

- Analysis

- Membrane expression via line profile analysis

- Cell count and transduction efficiency (initially, continued by B.B.)

- ABRs (with analysis toolbox of A.H.)

- Preparation of the manuscript (together with T.M., V.R., A.N.) 


\subsection{Abstract}

Optogenetic tools, providing non-invasive control over selected cells, have the potential to revolutionize sensory prostheses for humans. Optogenetic stimulation of spiral ganglion neurons (SGNs) in the ear provides a future alternative to electrical stimulation used in cochlear implants. However, most channelrhodopsins do not support the high temporal fidelity pertinent to auditory coding because they require milliseconds to close after light-off. Here, we biophysically characterized the fast channelrhodopsin Chronos and revealed a deactivation time constant of less than a millisecond at body temperature. In order to enhance neural expression, we improved its trafficking to the plasma membrane (Chronos-ES/TS). Following efficient transduction of SGNs using early postnatal injection of the adeno-associated virus AAV-PHP.B into the mouse cochlea, fiber-based optical stimulation elicited optical auditory brainstem responses (OABR) with minimal latencies of $1 \mathrm{~ms}$, thresholds of $5 \mu \mathrm{J}$ and $100 \mu$ s per pulse, and sizable amplitudes even at $1000 \mathrm{~Hz}$ of stimulation. Recordings from single SGNs demonstrated good temporal precision of light-evoked spiking. In conclusion, efficient virus-mediated expression of targeting-optimized Chronos-ES/TS achieves ultrafast optogenetic control of neurons.

\subsection{Introduction}

Since the discovery of channelrhodopsins (ChRs, (Nagel et al, 2002, 2003) and the application of these light-gated ion channels for controlling excitable cells (Boyden et al, 2005), the concept of optogenetics has revolutionized the life sciences (Adamantidis et al, 2015; Kim et al, 2017). Application of optogenetics to restore sensory function in the immune-privileged eye and the ear are thought to have a fair chance of clinical translation (Jeschke \& Moser, 2015; Sahel \& Roska, 2013). Indeed, AAVmediated optogenetics for vision restoration has recently entered a first clinical trial in a dose-finding effort (RST-001 Phase I/II Trial for Advanced Retinitis Pigmentosa - Full Text View - ClinicalTrials.gov). While neural coding of visual information can likely be achieved with ChRs that deactivate within several milliseconds (Busskamp et al, 2012), faster ChRs are required for sound coding in spiral ganglion neurons of the ear (SGNs) that spike at hundreds of $\mathrm{Hz}$ with sub-millisecond precision (Jeschke \& Moser, 2015).

If such ultrafast optogenetic control of neural activity was available, it would serve auditory research and could fuel the development of future optical cochlear implants (oCls). Clinically, this is highly relevant as approximately 360 million people $-5 \%$ of the world's population - suffer from a disabling hearing impairment (WHO, 2006) and we are still lacking causal therapies for the most common form; sensorineural hearing impairment. Consequences are impaired communication, often social isolation, depression, and reduction in professional capabilities. As of today, partial restoration of auditory function by hearing aids and electrical $\mathrm{Cls}(\mathrm{eCl})$ represent the options of choice for rehabilitation in 
sensorineural hearing impairment, which results from cochlear dysfunction or degeneration. The eCl bypasses dysfunctional or lost cochlear hair cells via direct electric stimulation of SGNs and, with most of the approximately 500.000 users achieving open speech comprehension, is considered the most successful neuroprosthesis (Zeng, 2017; Lenarz, 2018). Nonetheless, there is an urgent need for further improvement of the $\mathrm{Cl}$. The biggest bottleneck of the $\mathrm{eCl}$ is the poor spectral resolution of coding that arises from the wide spread of current around each electrode contact (Kral et al, 1998). Using light for stimulation in $\mathrm{oCl}$ is one of the present developments to improve spectral coding by $\mathrm{Cls}$, as light can be better spatially confined than electric current (e.g. Richter et al, 2011; Hernandez et al, 2014). One of the implementations used optogenetic stimulation of SGNs for a first proof-of-principle study on activation of the auditory pathway up to the midbrain (inferior colliculus, IC), demonstrating a lower spread of cochlear excitation for fiber-based oCl than for monopolar eCl (Hernandez et al, 2014). However, the temporal fidelity of ChR2-mediated optogenetic control of SGN firing seemed limited; auditory brainstem responses broke down even below $100 \mathrm{~Hz}$ of stimulation. Higher temporal fidelity of optogenetic SGN stimulation might be achieved when using faster ChRs such as Chronos (Klapoetke et al, 2014) or the newly engineered Chronos mutant ChroME (Mardinly et al, 2018).

Therefore, characterizing and optimizing fast ChRs is of great importance for fast spiking neurons in the auditory system, but also in the somatosensory system, cerebellum, and a wide range of inhibitory circuits. Here, we targeted Chronos, the fastest ChR reported so far, and first dissected its gating by patch-clamp recordings of photocurrents. Towards its application for optogenetic stimulation of mouse SGNs we optimized Chronos, the viral vector and virus injection approach for achieving high plasma membrane expression. As described for another opsin (Gradinaru et al, 2010) we appended sequences for improved exit from the endoplasmic reticulum (ES) (Stockklausner et al, 2001; Ma et al, 2001) and trafficking to the plasma membrane (TS) (Hofherr, 2005) to Chronos (Chronos-ES/TS) and performed postnatal injections of AAV-PHP.B serotype (Deverman et al, 2016), which drove highly efficient Chronos-ES/TS expression. We demonstrate by recordings of oABR and single SGN firing that Chronos-ES/TS enables ultrafast stimulation of the auditory pathway. 


\subsection{Results}

\subsubsection{Chronos undergoes sub-millisecond on/off transitions at $36^{\circ} \mathrm{C}$}

The kinetic properties of ChRs are best studied in cells with little background conductance to characterize the light-induced conductance in isolation. To this end, we used Human Embryonic Kidney cells 293 T (HEK-293T cells) expressing Chronos or ChR2 (Methods). We clamped the membrane voltage to $-60 \mathrm{mV}$ where any light-induced change in the conductance is then linearly reflected in a change of the pipette current. First, we compared gating kinetics of ChR2 and Chronos at a low light intensity of $0.27 \mathrm{~mW} \mathrm{~mm}^{-2}$, far below the half maximal activation. At $22^{\circ} \mathrm{C}$, we found activation and de-activation time constants (mean \pm SEM) of $\tau_{\text {act }}=4.9 \pm 0.3 \mathrm{~ms}, \tau_{\text {deact }}=9.4 \pm 1.0 \mathrm{~ms}(n=6)$ for ChR2 and $\tau_{\text {act }}=1.5 \pm 0.1 \mathrm{~ms}, \quad \tau_{\text {deact }}=3.0 \pm 0.2 \mathrm{~ms}(\mathrm{n}=21)$ for Chronos (Figure $\left.1 \mathrm{~A}\right)$. When increasing the temperature to $36^{\circ} \mathrm{C}$, activation and deactivation accelerated, with $\tau_{\text {act }}=0.58 \pm 0.02 \mathrm{~ms}$ and $\tau_{\text {deact }}=0.76 \pm 0.05 \mathrm{~ms}(\mathrm{n}=6)$, Chronos reached the sub-millisecond range, while ChR2 gating kinetics, $\tau_{\text {act }}=2.3 \pm 0.1 \mathrm{~ms}, \tau_{\text {deact }}=3.0 \pm 0.3 \mathrm{~ms}$ ( $\mathrm{n}=6$ and 5 respectively), at $36^{\circ} \mathrm{C}$ were comparable to the values achieved by Chronos at $22^{\circ} \mathrm{C}$. Hence, already at such a low light intensity, Chronos, but not ChR2, activation and deactivation should permit signal transmission with a bandwidth of several hundred Hertz. Probing the frequency bandwidth directly by applying light chirps, Chronos confers a much higher bandwidth compared to ChR2 (Figure 1B). The gain of chirp responses could be very well characterized by a single cut-off frequency of $24 \mathrm{~Hz}$ and $86 \mathrm{~Hz}$ for ChR2 and Chronos, respectively, at $22^{\circ} \mathrm{C}$ and $63 \mathrm{~Hz}$ and $150 \mathrm{~Hz}$ at $36^{\circ} \mathrm{C}$ (Figure $1 \mathrm{C}$ ). 

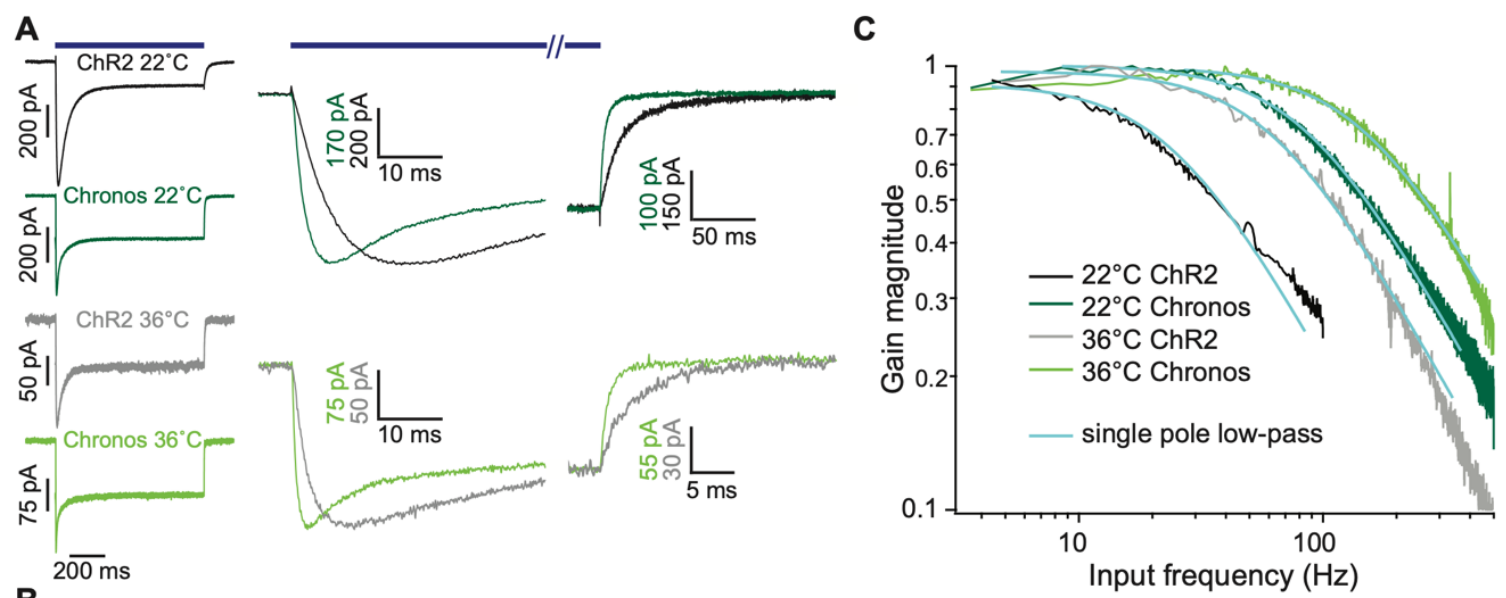

B
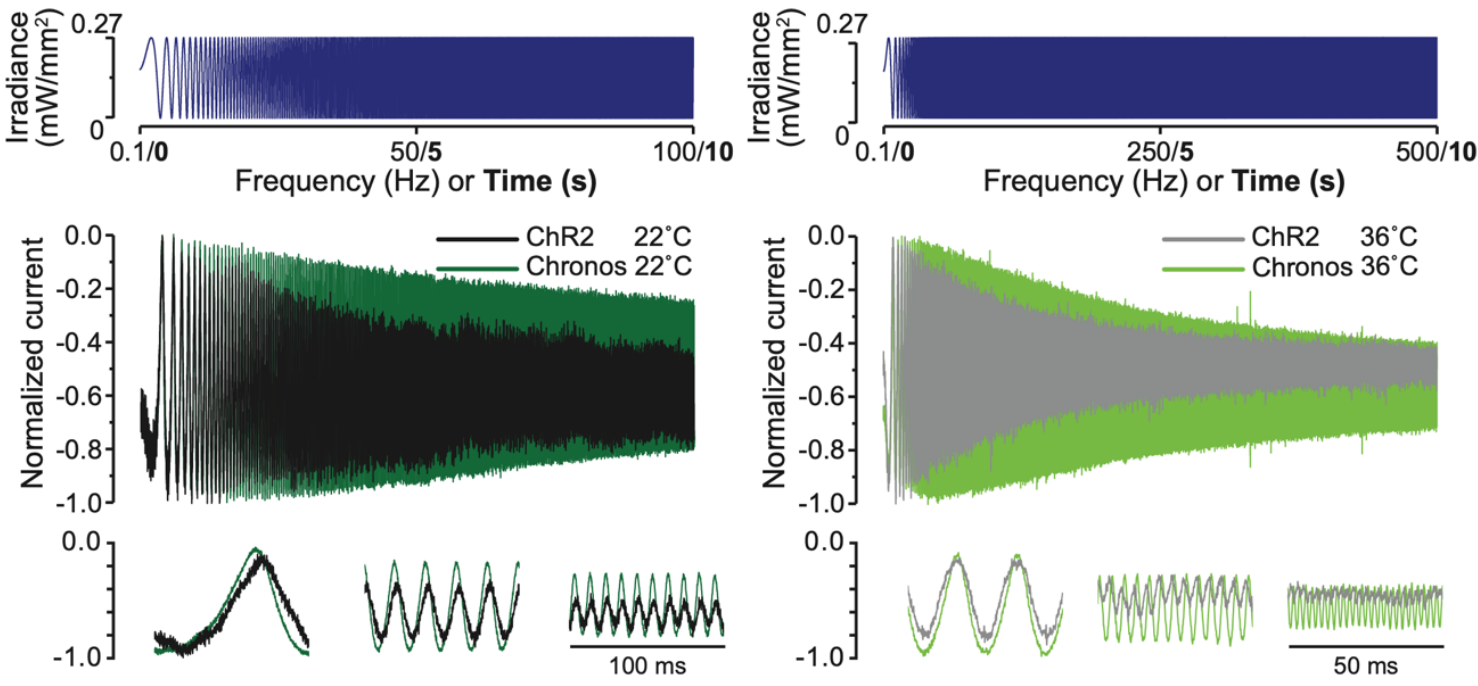

Figure 1. Chronos mediates fast light-driven currents

(A) One second long light pulses (LED centered $480 \mathrm{~nm}, 0.27 \mathrm{~mW} \mathrm{~mm}^{-2}$ ) elicit current responses in HEK$293 \mathrm{~T}$ cells expressing ChR2 and Chronos, tested at $22^{\circ} \mathrm{C}$ and $36^{\circ} \mathrm{C}$. Right panels show activation and deactivation at higher time resolution.

(B) A quasi-periodic chirp stimulus is use to directly probe the bandwidth of Chronos- and ChR2mediated photocurrents in HEK-293T cells. Top: stimulus, middle: full response, bottom: sections from the beginning, middle and end of the response. Note the substantially larger frequency range over which Chronos currents follow the light stimulus. At $36^{\circ} \mathrm{C}$ this range is extended even further.

(C) Analysis of the chirp responses of HEK-293T cells as in Figure 1B. The current amplitude modulation is plotted against the stimulus frequency. The smooth lines represent fits to the magnitude of the transfer function of a single pole filter $\operatorname{abs}\left(\left(1+i f / f_{\text {cut }}\right)^{-1}\right)$.

\subsubsection{Improving the plasma membrane expression of Chronos}

The above biophysical characterization had indicated Chronos as a strong candidate for optogenetic stimulation of SGNs with the required high temporal fidelity, provided sufficient plasma membrane expression can be achieved. Recent studies have shown that adding ER export and trafficking signals, isolated from a vertebrate inward rectifier potassium channel, to the cytoplasmic C-terminus of opsins promote their plasma membrane expression (Gradinaru et al, 2010). Hence, we added these sequences, here nick-named ES (Export Signal) and TS (Trafficking Signal), sandwiching EYFP, to Chronos (Chronos-ES/TS, Figure 2A). We first compared expression of Chronos-ES/TS and Chronos in 
HEK-293T cells transfected with the respective pAAV plasmid by immunocytochemistry. Using confocal and stimulated emission depletion (STED) microscopy we found a preferentially peripheral, likely plasmalemmal, localization of Chronos-ES/TS with some intracellular signal most likely arising from the Golgi, while the original Chronos construct was more diffusely distributed throughout the extranuclear intracellular space (Figure 2B-D). We quantified the cellular distribution of the opsin by line profile analysis (Figure 2C) and found a significantly greater peripheral than central immunofluorescence for Chronos-ES/TS when compared to the original Chronos construct (Figure 2C, D). The larger variance of the ratio of membrane and intracellular fluorescence for Chronos-ES/TS (Figure 2D) is likely explained by dividing by the relatively low, yet varying intracellular abundance of the opsin. In summary, the data suggests improved trafficking to the plasma membrane of ChronosES/TS.

A
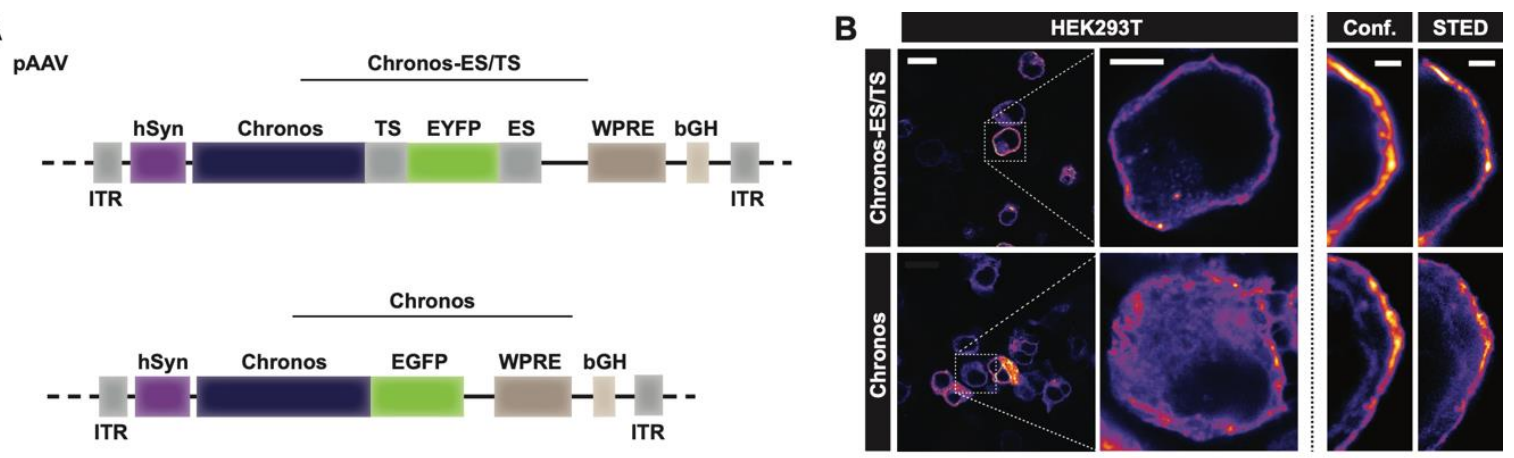

C
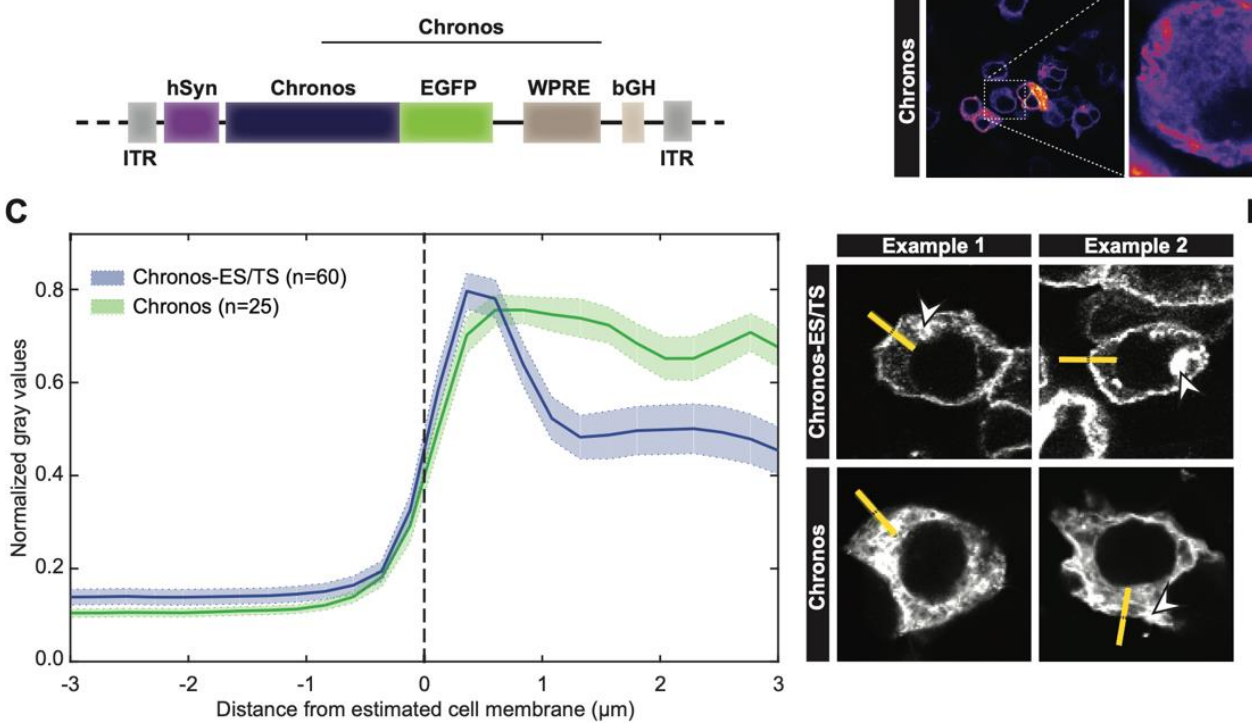

D

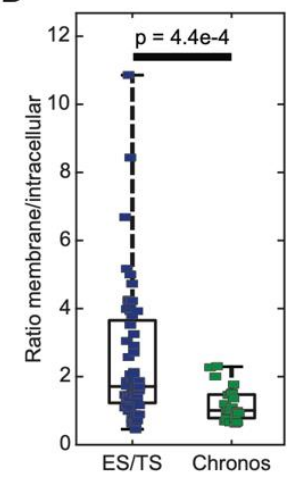

Figure 2. Optimizing membrane expression of Chronos by adding ER-exit and trafficking signals: HEK293T cells

(A) pAAV vector used in the study Chronos with a trafficking signal (TS), EYFP and ER export signal (ES) Chronos-ES/TS (upper) or containing the original Chronos-EGFP (Klapoetke et al, 2014), lower). In each, expression was driven by the human synapsin promoter (hSyn) and enhanced by the Woodchuck hepatitis virus posttranslational regulatory element (WPRE) and bovine Growth Hormone (bGH) polyadenylation signal ( $\mathrm{bGH}$ poly $\mathrm{A}$ ) sequences. ITR: inverted terminal repeats

(B) Confocal and STED section of representative HEK-293T cells transfected with ChronosES/TS (upper) and Chronos (lower) and immunolabeled for FP: membranous labeling is more obvious for ChronosES/TS. Scale bars: 10,5 and $2 \mu \mathrm{m}$ for left, middle and right panels.

(C) Peak-normalized line profiles $(7.5 \mu \mathrm{m})$ centered on the estimated membrane of HEK-293T cells expressing Chronos-ES/TS (blue) or Chronos (green) as in (B): mean \pm SEM. Chronos-ES/TS-expressing cells showed a clear peripheral, likely membrane peak, which is missing in Chronos-expressing cells. Right panels show exemplary line profile placements (yellow). One line per cell was placed 
perpendicular and centered to cell edge, aiming at sufficient intracellular coverage and avoiding fluorescent aggregates (arrowheads). $\mathrm{N}$ corresponds to analyzed cells (1 sample/cell).

(D) Box and whisker plot ratio of maximal membrane and maximal cytoplasmic fluorescence of immunolabeled HEK-293T cells expressing Chronos-ES/TS or Chronos: Mann-Whitney U test showed significantly higher ratio in Chronos-ES/TS cells demonstrating an improved membrane expression of Chronos-ES/TS ( $p$-value $=4.4 \mathrm{e}-4$ ). The horizontal line within the box indicates the median, boundaries of the box indicate the 0.25- and 0.75-percentile, and the whiskers indicate the highest and lowest values of the results. Squares: individual data points. For details on membranous and cytoplasmic area see method section.

Similar findings were also obtained in hippocampal neurons in culture transduced by AAV2/6 or AAVPHP.B (Figure 3). This indicates that neurons, too, struggle to traffic Chronos to the plasma membrane and that this can be alleviated when adding the ES- and TS-signals, at least when tested in culture.

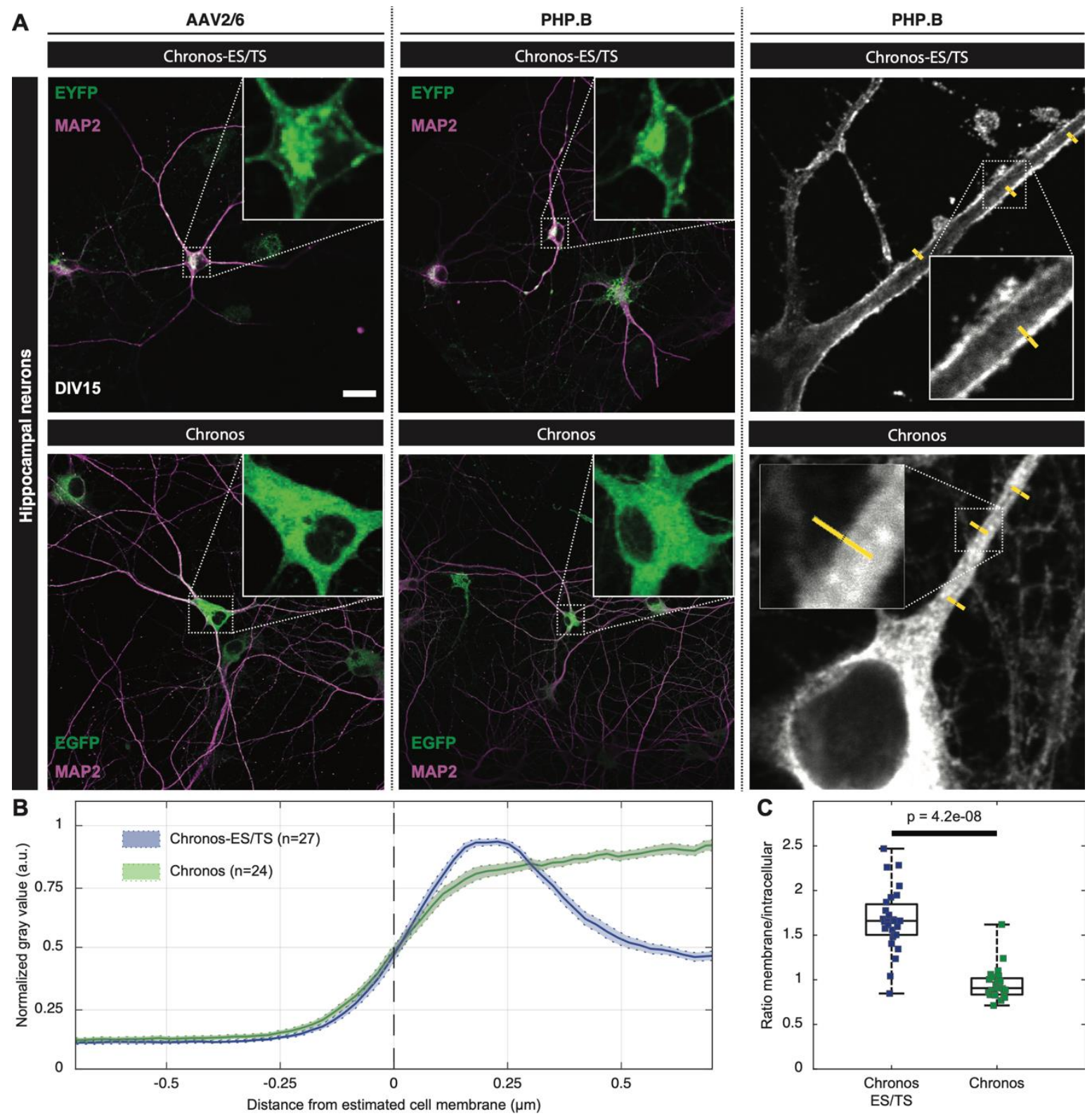

Figure 3. Improved membrane expression of optimized Chronos-ES/TS in hippocampal neurons

(A) Hippocampal neurons infected at DIV 10 with two different versions of Chronos show very distinct expression patterns. Neurons infected with Chronos-ES/TS (upper panels) either using AAV2/6 (left) or 
PHP.B virus (middle) show very specific plasma membrane expression in somatic regions and proximal dendrites. Infection of neurons with Chronos (lower panels) either using AAV2/6 (left) or AAV-PHP.B (middle) showed more intracellular opsin abundance. Right panels show exemplary line profile placement (yellow). Scale bar: $50 \mu \mathrm{m}$ applies to all panels.

(B) Peak-normalized line profiles $(1.5 \mu \mathrm{m})$ centered on the outer cell edge of AAV-PHP.B transduced hippocampal proximal dendrites expressing Chronos-ES/TS (blue) or Chronos (green) as in (A): mean \pm SEM. Chronos-ES/TS-expressing cells showed a clear peripheral, likely plasmalemmal peak, which is missing in Chronos-expressing cells.

(C) Box and whisker plot of the ratio of maximal membrane and maximal intracellular fluorescence of immunolabeled hippocampal neurons expressing Chronos-ES/TS or Chronos: Mann-Whitney U test showed significantly higher ratio in Chronos-ES/TS cells demonstrating an improved membrane expression of Chronos-ES/TS ( $p$-value $=4.2 \mathrm{e}-8$ ). The horizontal line within the box indicates the median, boundaries of the box indicate the 0.25 - and 0.75 -percentile, and the whiskers indicate the highest and lowest values of the results. Squares: individual data points. For details on membranous and cytoplasmic area see method section.

\subsubsection{AAV-mediated expression of Chronos and Chronos-ES/TS in mouse SGNs}

Next, we turned to expression of Chronos in mouse SGNs in vivo and, once more, compared the original Chronos and Chronos-ES/TS. We aimed to establish efficient AAV-mediated transduction of SGNs and employed the human synapsin promoter (hSyn, Figure 2A) that had turned out to drive efficient and specific SGN expression (Hernandez et al., 2014). We first followed our previous protocol using transuterine injections of AAV2/6 into the otocyst of mouse embryos at embryonic day 11.5 (Figure 4A, upper). In most of the cases the expression of Chronos-EGFP was absent or sparse (Figure 4B, left and middle). Exceptionally we saw high expression levels (Figure 4B right). As before (Hernandez et al., 2014), the expression, if any, was largely limited to the SGNs of the basal cochlear turn and was never seen in inner hair cells (Figure 4B right, inset).

Next, we moved on to early postnatal injections (Figure 4A, middle and lower, postnatal day 5-7) into the cochlea via the round window, which had proven highly successful for transduction of hair cells (e.g. Akil et al., 2012; Jung et al., 2015). We employed AAV-PHP.B, a novel AAV-serotype (Deverman et $a l, 2016)$ with improved efficiency of neural transduction, for expression of Chronos-ES/TS and Chronos (hSyn promoter, comparable titers, $10^{12} \mathrm{GC} \mathrm{ml}^{-1}$ ) in SGNs. 12 out 12 AAV-PHP.B-injected mice showed substantial SGN transduction in immunohistochemistry for Chronos-ES/TS and 9 out 9 for Chronos. Postnatal injection of AAV-PHP.B drove strong expression of Chronos-ES/TS across all cochlear turns (Figure 4C). Similar to HEK-293T cells and hippocampal neurons in vitro, Chronos-ES/TS localized preferentially peripheral, likely plasmalemmal in SGNs in vivo, while the original Chronos construct was more diffusely distributed throughout the cell (Figure 4D). Once again, we quantified the cellular distribution of the opsin by line profile analysis (Figure 4E) and found a significantly greater peripheral than central immunofluorescence for Chronos-ES/TS when compared to the original Chronos construct (Figure $4 \mathrm{E}, \mathrm{p}=4.1 \mathrm{e}-5$ ). The difference can be appreciated by the $0.56 \mu \mathrm{m}$ more intracellular $50 \% \mathrm{FP}$ immunofluorescence for Chronos. We note that the cytosolic parvalbumin immunofluorescence 
allowed a better estimation of the cell border which was independent of the opsin expression, both advantageous when compared to HEK-293T cell analysis (Figure 2). Despite some differences in absolute numbers between both analyses, which are not unexpected given the different cell types, means of transfection and analysis method, both support the main observation: improved relative plasma membrane abundance of Chronos-ES/TS.

A
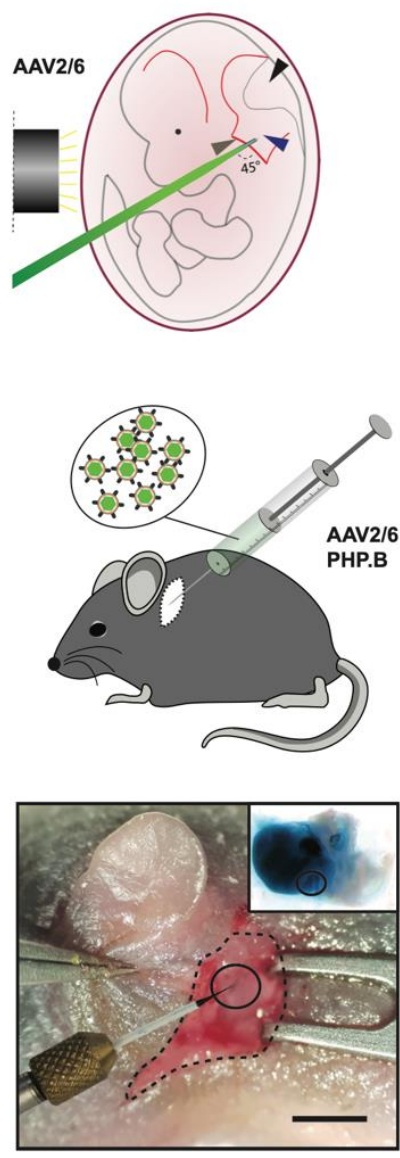

E 1.0

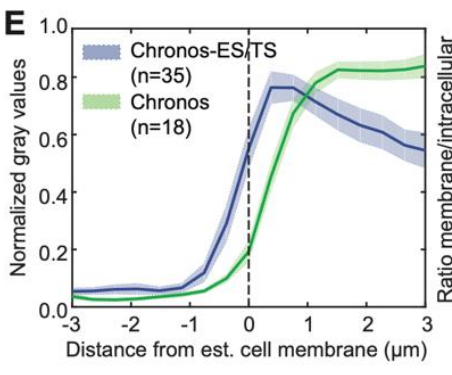

B

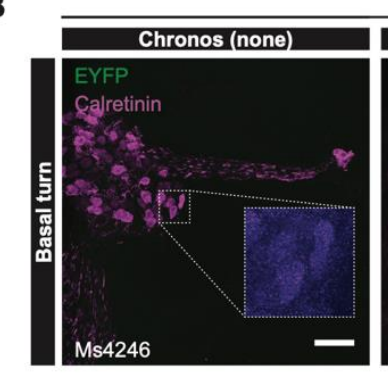

C

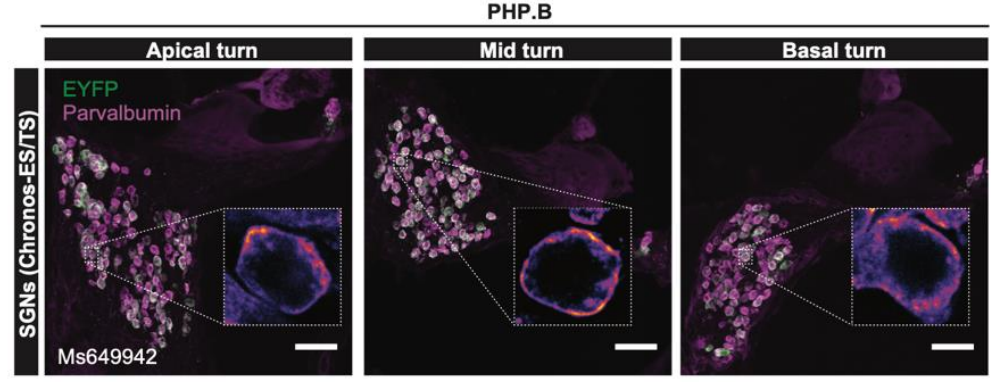

D
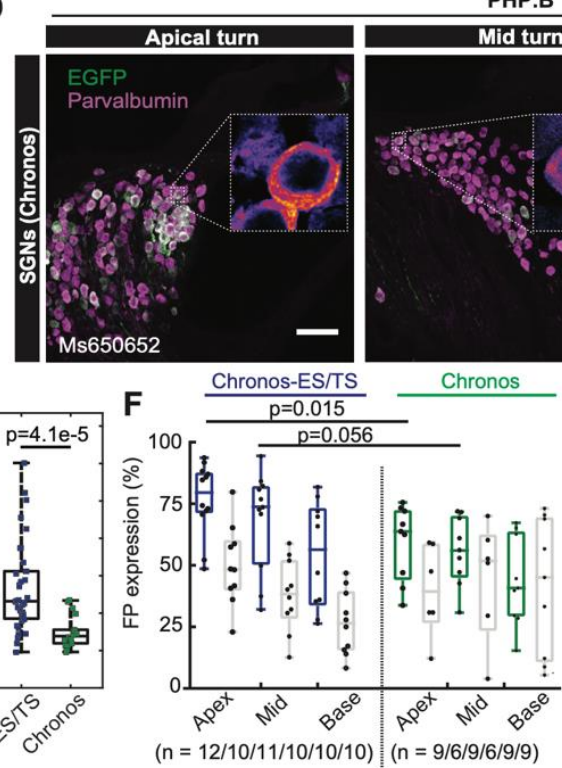

AAV2/6

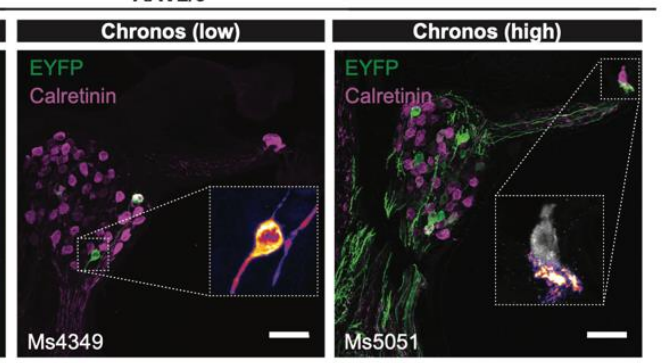

PHP.B

PHP.B
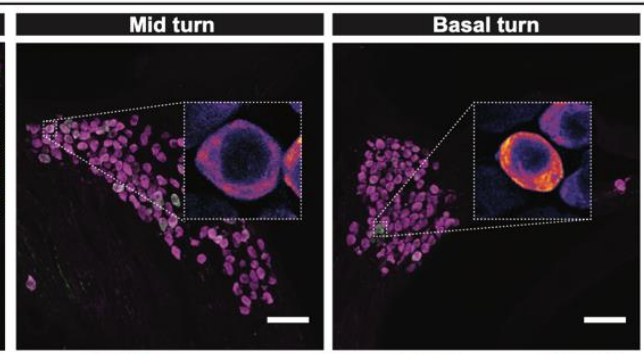

Chronos-ES/TS Chronos

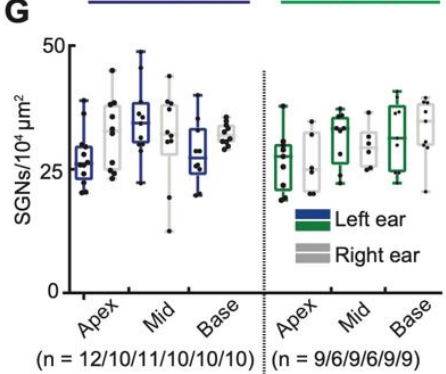

Figure 4. Establishing efficient expression of Chronos in SGNs: use of Chronos-ES/TS, potent AAVPHP.B, and postnatal mode of AAV-injection

(A) Upper panel: schematic representation of the viral injection into the embryonic otocyst (left: black cylinder marks the light guide used to trans-illuminate the embryo in the uterus after mobilization from the abdominal cavity, green: micropipette filled with fast green-colored AAV suspension). Middle panel: schematic representation of AAV-injection into the postnatal cochlea via the round window (RW). Lower panel: surgical situs of a $\mathrm{p} 7$ mouse with retroauricular incision, graphical aid encircles the injection site). Inset shows ex-vivo cochlea just after AAV injection via RW. Scale bar: $2 \mathrm{~mm}$.

(B) Maximum projection of confocal images of immunolabeled mid-modiolar cochlear cryosections (exemplary sections of basal turn) of embryonically AAV2/6-Chronos-injected mice collected at 4- 
weeks of age. EYFP (green) marks transduced SGNs, calretinin (magenta) was used as generic marker of SGNs, scale bar: $50 \mu \mathrm{m}$. In the inset, color code for EYFP channel was changed to fire (EYFP). Left panel: most common, non-expressing example, inset shows zoom of negative SGNs. Middle panel: occasional, sparsely expressing example, inset: one out of two positive SGNs. Right panel: rare, highly expressing example, inset: negative inner hair cell (calretinin in gray), exclusive localization of EYFP in the SGN boutons and fibers.

(C) Postnatally AAV-PHP.B-Chronos-ES/TS-injected mouse (see (B) except where stated differently). EYFP (green) marks transduced SGNs, parvalbumin (magenta) was used as generic marker of SGNs, scale bar: $50 \mu \mathrm{m}$. High transduction rate, good membrane expression. In inset, color code for the green channel was changed to fire for better visualization. Similar to (B).

(D) Postnatally AAV-PHP.B-Chronos-injected mouse (see (C) except where stated differently). Substantial SGN transduction, poor membrane expression.

(E) Line profile analysis of FP-immunofluorescence across the membrane of SGN somata. Traces were centered at the transition from high to low parvalbumin immunofluorescence as a proxy of plasma membrane location. Left panel: clear membrane abundance in Chronos-ES/TS (blue, mean \pm SEM), but mostly intracellular localization in Chronos (green, mean \pm SEM). Right panel: Box and whisker plot of the ratio of maximal membrane and maximal intracellular FP fluorescence for Chronos-ES/TS (left) and Chronos (right): stronger relative membrane expression in Chronos-ES/TS (Mann-Whitney U test, pvalue $=4.1 \mathrm{e}-5)$. Squares: individual data points. For details on membranous and cytoplasmic area see method section.

(F) Box and whisker plot of the fraction of FP-positive SGNs (transduced out of all parvalbumin-positive SGNs) for the apical, middle and basal cochlear turn of the injected ear (colored) and contralateral, non-injected (grey) ear of Chronos-ES/TS-AAV-PHP.B and Chronos-AAV-PHP.B mice. Points: individual animals plotted on top; $\mathrm{n}$ refers to number of cochleae studied. The horizontal line within the box indicates the median, boundaries of the box indicate the 0.25 - and 0.75 -percentile, and the whiskers indicate the highest and lowest values of the results.

(G) Box and whisker of the SGN density for the apical, middle and basal cochlear turn of the injected (colored) ear and the contralateral, non-injected (grey) ear (as in (F)). No significant differences between Chronos and Chronos-ES/TS nor between injected and non-injected ear. Points: individual animals plotted on top; $\mathrm{n}$ refers to number of cochleae studied.

When analyzing the transduction rates in the injected (left) and non-injected (right) cochleae, we confirmed robust SGN transduction across all turns of the injected cochlea for both Chronos-ES/TS and Chronos (Figure 4F). We note that the counterstain for parvalbumin- $\alpha$, used for AAV-PHP.B injected ears, is a more general marker of SGNs than calretinin, used for AAV2/6 injected ears, which is present only in a subset of SGNs. Therefore, if anything, we would have been prone to overestimate the transduction rate for AAV2/6 injected ears, which, however, was very low. Interestingly, for both cases of AAV-PHP.B injection we also found substantial expression in the contralateral, non-injected cochleae, indicating spread of virus in the specific conditions of pressure injection into the scala tympani of the early postnatal cochlea. This spread likely occurred via the cochlear aqueduct and/or the endolymphatic ducts and the cerebrospinal fluid space (Lalwani et al, 1996). The density of SGNs in the injected as well as in non-injected ears were comparable (Figure 4G). The injected mice behaved normal as concluded from routine animal observation. 
2.3.4 Chronos-mediated optogenetic neural stimulation of the auditory pathway: transuterine injections of $A A V 2 / 6$

We performed acute recordings of oABRs using fiber-coupled laser stimulation 4-12 weeks after AAVinjection. Following posterior tympanotomy, we inserted a $50 \mu \mathrm{m}$ optical fiber through the round window (RW) to project the light of a blue laser $(\lambda=473 \mathrm{~nm})$ onto the SGNs. When oABRs were present (Figure EV1), we typically found three to five OABR peaks, which likely reflected the synchronous activation of Chronos-expressing SGNs (first peak) and downstream auditory pathway (subsequent peaks). Transuterine injections of AAV2/6-Chronos did not generally support oABRs; only 3 out of 120 injected mice (including 2 different AAV2/6 produces) showed oABRs. In these positive mice, OABR grew in amplitude and showed shorter latencies when increasing radiant flux (see inset of Figure EV1).

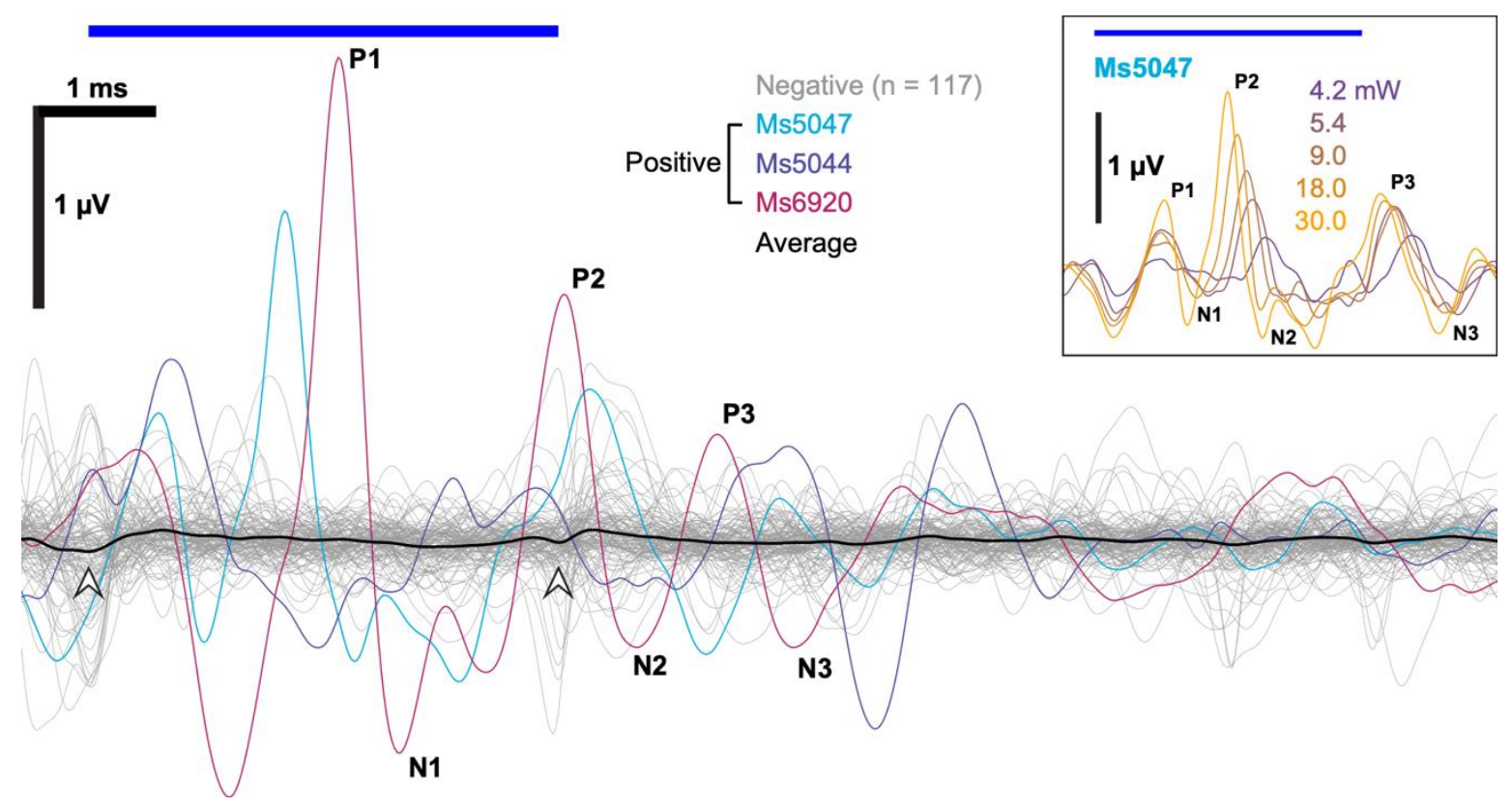

Figure EV1. Demonstrating functional expression of Chronos in SGNs following transuterine AAV2/6 injection reported by recordings of OABRs

In 3 out of 120 animals we could record oABRs using fiber-coupled laser stimulation 4-12 weeks after embryonic transuterine injection of AAV2/6 carrying Chronos using 4 ms pulses at $10 \mathrm{~Hz}$ with $30 \mathrm{~mW}$ radiant flux. Responses were verified as $O A B R s$ by the increasing amplitude with stronger light pulses (inset: shows oABRs of an exemplary oABR-positive mouse for varying radiant flux, colors code the radiant flux in $\mathrm{mW}$ ). A small onset and offset artifact (arrowheads) is visible for the average (black) of the negative animals (gray, $n=117$ ) which we occasionally observed in the early phase of the project.

\subsubsection{Chronos-ES/TS enables ultrafast optogenetic stimulation of the auditory pathway: OABRs}

Postnatal injection of AAV-PHP.B reliably achieved high transduction rates (Figure 4), which enabled OABRs in 19 out of 20 AAV-PHP.B-Chronos-ES/TS-injected mice and 8 out of 17 AAV-PHP.B-Chronosinjected mice. Next, we performed a detailed characterization of Chronos-ES/TS-mediated optogenetic activation using OABRs and compared the results to those obtained with Chronos. OABR amplitude grew with increasing stimulus intensity (Figure 5A, B, 1 ms light pulses delivered at $10 \mathrm{~Hz}$ - averaged 
across 1000 repetitions), while oABR latency (i.e. the time interval between the stimulus onset and the OABR $\mathrm{P}_{1}$ wave) got shorter for Chronos-ES/TS (sign test between the threshold and highest radiant flux tested, $p$-value $=0.009$ ) and was constant for Chronos (Figure 5A, C; $p$-value $=0.11$ ). oABR thresholds and amplitudes differed between animals (shown for the first peak, $\mathrm{P}_{1}-\mathrm{N}_{1}$, Figure $5 \mathrm{~B}$ ). Stimuli as weak as $1.08 \mathrm{~mW}$ (duration: $1 \mathrm{~ms}$, rate: $10 \mathrm{~Hz}$ - averaged across 1000 repetitions) were sufficient to drive oABRs in one Chronos ES/TS injected mouse and $4.56 \mathrm{~mW}$ in a Chronos injected mouse. For $1 \mathrm{~ms}$ light pulses delivered at $10 \mathrm{~Hz}$, the average $O A B R$ threshold amounted to $6.58 \pm 1.08 \mathrm{~mW}$ and $13.95 \pm 3.52 \mathrm{~mW}$ for Chronos-ES/TS and Chronos, respectively ( $p$-value $=0.0367$, Mann-Whitney U test; $n=13$ and $n=8$ ). In most animals oABR amplitudes grew with radiant flux increasing over more than one order of magnitude (Figure $5 B$ ). The minimal latency of the first oABR peak ( $P_{1}$, Figure $5 C$ ) amounted to $0.95 \pm 0.07 \mathrm{~ms}(\mathrm{n}=13)$ for Chronos-ES/TS and $1.26 \pm 0.07 \mathrm{~ms}$ for Chronos $(\mathrm{n}=8, p$ value $=0.014$, Mann-Whitney $\mathrm{U}$ test $)$. 

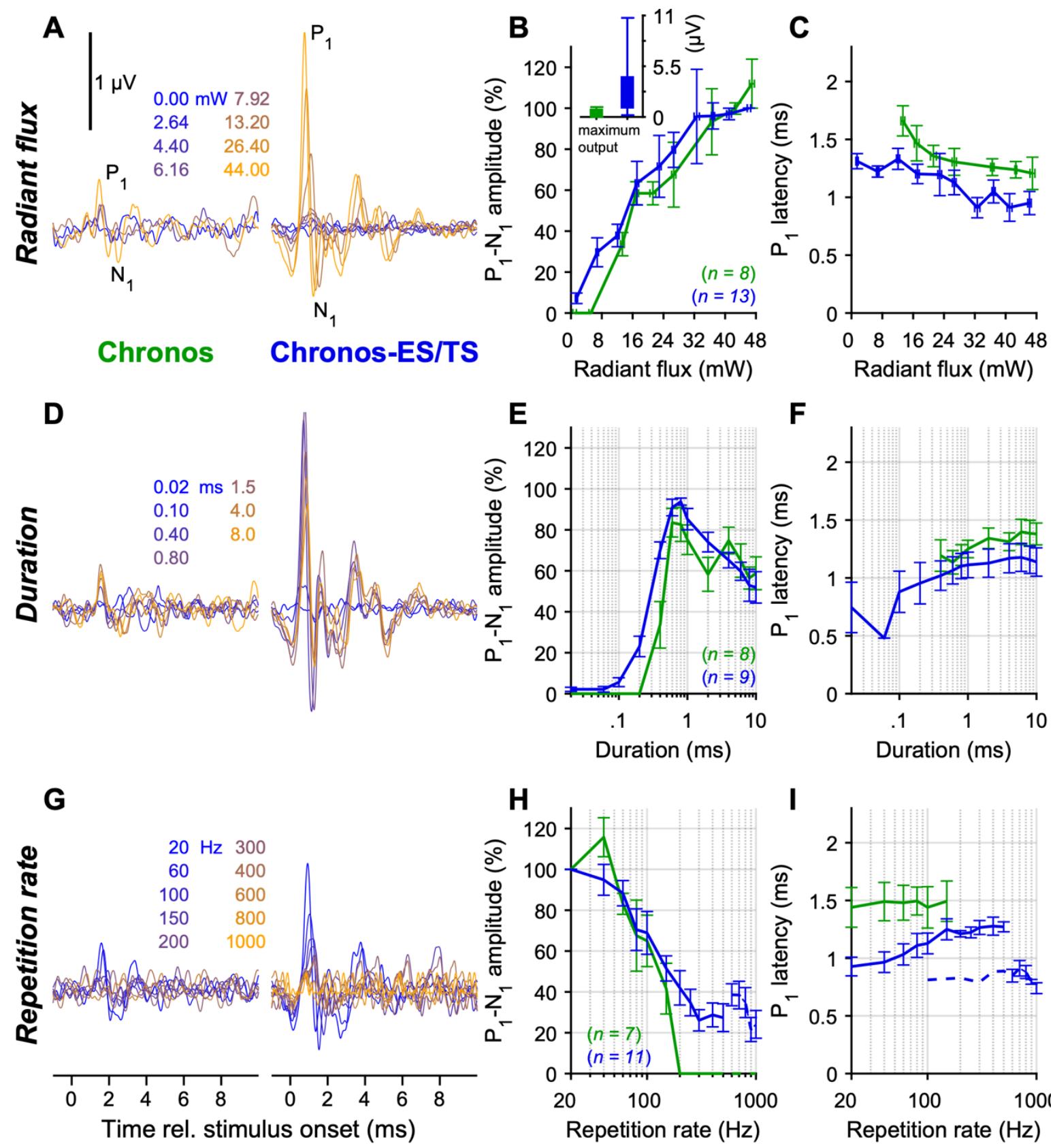

Figure 5. Characterizing optogenetic stimulation by recordings of $O A B R s$

(A) OABRs driven with varying radiant flux $(1 \mathrm{~ms}$ at $10 \mathrm{~Hz}$, colors code the radiant flux in $\mathrm{mW}$ ) for exemplary mice injected with AAV-PHP.B-Chronos (left) or AAV-PHP.B-Chronos-ES/TS (right).

(B) Normalized P1 - N1 amplitude of oABR as a function of light intensity for Chronos as in A (green, throughout figure) and Chronos-ES/TS (blue). Radiant flux was binned per $5 \mathrm{~mW}$ steps (horizontal error: \pm SEM). Inset: maximal absolute P1 $-\mathrm{N} 1$ amplitude of oABR (mean \pm SEM) was greater for Chronos-ES/TS than for Chronos (Mann-Whitney $U$ test, $p$-value $\leq 0.001$ ).

(C) Latency of oABR P1 as a function of radiant flux as in (B). Radiant flux was binned per $5 \mathrm{~mW}$ steps (horizontal error: \pm SEM).

(D) OABRs driven with varying stimulus duration $(10 \mathrm{~Hz}, 38 \mathrm{~mW}$, colors code the duration) for exemplary mice injected with AAV-PHP.B-Chronos (left) or AAV-PHP.B-Chronos-ES/TS (right).

(E) P1-N1 amplitude as a function of stimulus duration as in (D) (normalized against the largest P1-N1 oABR amplitude).

(F) Latency of $O A B R$ P1 as a function of stimulus duration as in (E). 
(G) OABRs driven with varying stimulus rate $(1 \mathrm{~ms}$ and $0.5 \mathrm{~ms}$ for $10-500 \mathrm{~Hz}$ and $500-1000 \mathrm{~Hz}$, respectively, $38 \mathrm{~mW}$, colors code the stimulus rate) for exemplary mice injected with AAV-PHP.BChronos (left) or AAV-PHP.B-Chronos-ES/TS (right).

(H) P1-N1 amplitude as a function of stimulus rate as in (G) (normalized against P1-N1 oABR amplitude at $20 \mathrm{~Hz}$ ).

(I) Latency of oABR P1 as a function of stimulus rate as in (H): Chronos-ES/TS enables responses up to at least $1000 \mathrm{~Hz}$. Dashed line indicates latencies data points obtained with a pulse duration of $0.5 \mathrm{~ms}$. The P1-N1 amplitude and P1 latency were measured on the same animals. Animal count (n) is stated on bottom right of panel $B, E$ and $H$. Data are expressed as mean \pm SEM.

oABRs could be elicited by light pulses as short as $20 \mu$ s with Chronos-ES/TS and $400 \mu s$ with Chronos (irradiance: $38 \mathrm{~mW}$, rate: $10 \mathrm{~Hz}$ - averaged across 1000 repetitions, Figure 5D, E). OABR amplitudes grew with pulse duration up to approximately $0.6 \mathrm{~ms}$ and tended to become smaller for longer pulses, possibly due to accumulating channel inactivation and/or increasing depolarization-block of SGNs upon prolonged photo-depolarization (Figure 5D, E).

Next, we tested the dependence of oABRs on the stimulus rate (duration: $1 \mathrm{~ms}$ up $500 \mathrm{~Hz}$ and $0.5 \mathrm{~ms}$ from $500 \mathrm{~Hz}$, intensity: maximum $(38-43 \mathrm{~mW}))$. When increasing stimulus rate, oABR amplitudes declined (Figure 5G) and latencies prolonged (Figure 5G, I). However, in contrast to our previous reports on : i) ChR2: where potentials were found only up to $60 \mathrm{~Hz}$ (Hernandez et al, 2014); ii) CatCh: up to $200 \mathrm{~Hz}$, and f-Chrimson: up to $250 \mathrm{~Hz}$ (respectively (Wrobel et al, 2018; Mager et al, 2018)); we could detect sizable $\mathrm{P}_{1}-\mathrm{N}_{1}$ up to stimulus rates of $500 \mathrm{~Hz}$ for Chronos (Figure $5 \mathrm{G} \mathrm{left,} \mathrm{H}$ ) and $1000 \mathrm{~Hz}$ for Chronos-ES/TS (the highest stimulus rate tested in our experiments, Figure 5G-H). $\mathrm{P}_{1}$-latency increased with higher stimulus rates in both cases.

\subsubsection{Chronos-ES/TS enables ultrafast optogenetic stimulation of the auditory pathway: recordings from single putative SGNs}

To further validate the Chronos-ES/TS-mediated SGN stimulation and evaluate the temporal fidelity of stimulation, we performed juxtacellular recordings from auditory nerve fibers (central axon of SGN) as described in (Hernandez et al, 2014; Mager et al, 2018). In brief, we targeted glass micropipettes to where the auditory nerve enters the anteroventral cochlear nucleus (AVCN) and searched for responses while stimulating the SGNs through the round window via an optical fiber coupled to a blue laser. We favored recordings of SGNs by deep positioning ( $>1000 \mu \mathrm{m}$ relative to the surface of the cochlear nucleus) of the pipette tip, but, given that responses to acoustic stimuli were lost upon the ear surgery, we could not safely discriminate SGNs from AVCN neurons, hence, we termed the lightresponsive neurons "putative SGN". We found that the putative SGNs fired upon optogenetic stimulation with high temporal precision for stimulus rates of up to hundreds of $\mathrm{Hz}$ (Figure 6A, B); some neurons followed stimulation to some extent even up to $1000 \mathrm{~Hz}$ (Figure 6B). Temporal precision of firing, evaluated based on vector strength (Goldberg \& Brown, 1969), see methods, Figure 6C) and 
spike jitter (i.e., standard deviation of spike latency across trials, Figure 6D), was generally high, but varied across the recorded neurons.

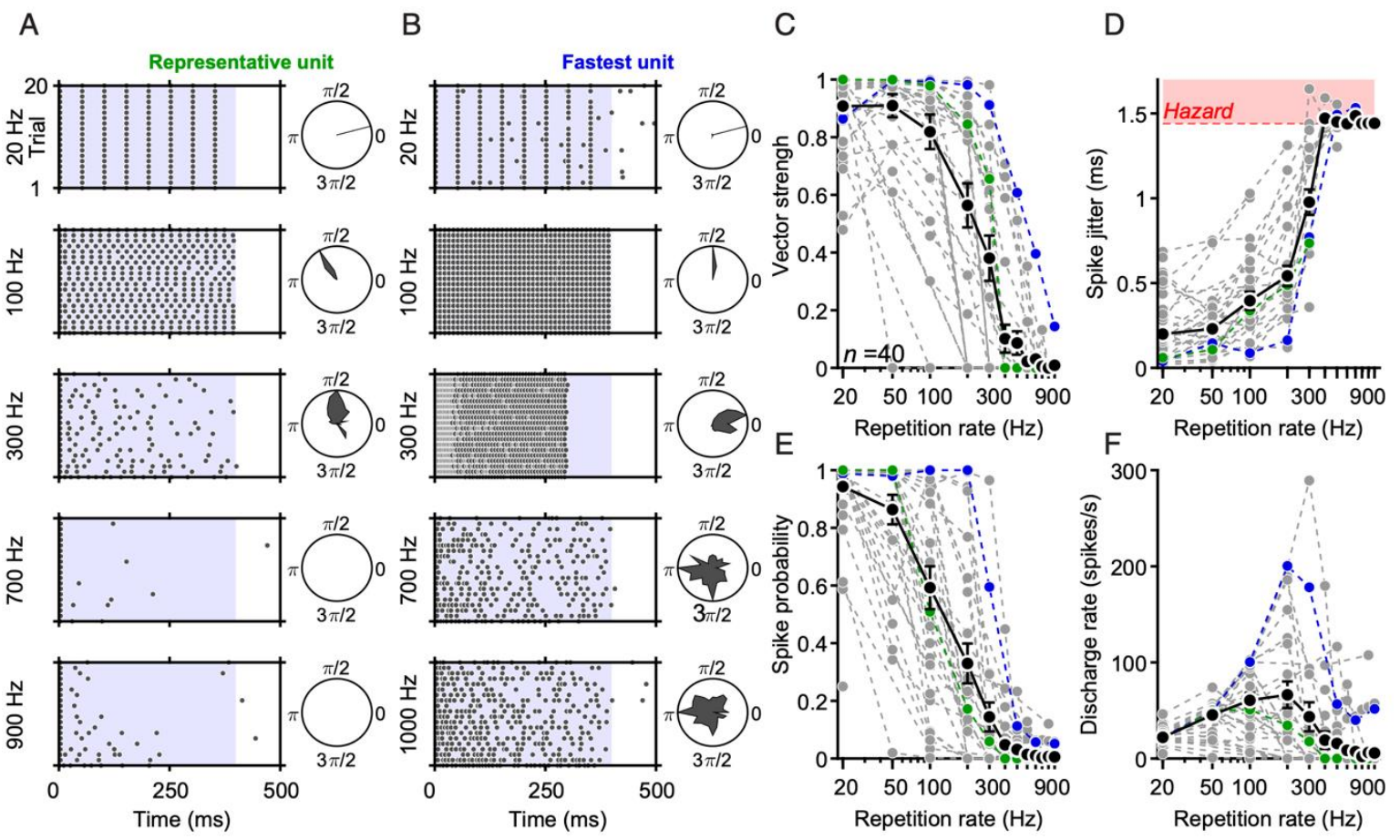

Figure 6. Characterizing optogenetic stimulation by extracellular recordings from single putative SGNs

(A) Raster plots showing spiking activity from a representative putative SGN ("unit") in response to 400 ms-long trains of laser pulses (at $30 \mathrm{~mW}, 1 \mathrm{~ms}$ for $20-600 \mathrm{~Hz}, 500 \mu \mathrm{s} \mathrm{for} \geq 700 \mathrm{~Hz}$ ) recorded at five different stimulation rates over 20 repetitions. Polar plots to the right side of raster plots show synchronicity of firing relative to the cycle between two pulse onsets.

(B) Same protocol and analysis of the "fastest unit" recorded, showing lower adaptation and firing even at very high stimulation rates.

(C-F) Quantification of the vector strength (C), spike jitter (D), spike probability (E) and discharge rate (F) as a function of repetition rate of 40 putative SGNs (obtained from 6 mice). In panel D the redshaded area represents the hazard function obtained in response to simulated Poisson spike trains.

Black data points and lines show mean \pm SEM, individual units are shown in gray. The units shown on panels $A-B$ are shown in green and blue, respectively. Number of units tested for each repetition rate: $20 \mathrm{~Hz}: 40,50 \mathrm{~Hz}: 25,100 \mathrm{~Hz}: 24,200 \mathrm{~Hz}: 26,300 \mathrm{~Hz}: 21,400 \mathrm{~Hz}: 20,500 \mathrm{~Hz}: 21,600 \mathrm{~Hz}: 18,700 \mathrm{~Hz}: 18$, $800 \mathrm{~Hz}:$ 19, $900 \mathrm{~Hz}: 21,1000 \mathrm{~Hz}: 19$.

Temporal precision and spike probability (Figure 6E) diminished with increasing stimulation rates, indicating that single SGNs code optogenetic information in a less reliable manner at very high stimulation rates. Spike jitter, calculated for spikes occurring in a time window comprised between two pulse onsets, increased with rate but was typically below a millisecond for stimulus rates lower than $300 \mathrm{~Hz}$ (Figure 6D). At higher stimulus rates, spike jitter increased beyond the values obtained for simulated Poisson spike trains (see methods, red-shaded area, Figure 6D), indicating spike synchronization with the light pulses became less reliable. The lower spike precision and limited spike probability at stimulus rates beyond $100 \mathrm{~Hz}$ observed are likely compensated by the population 
response, as several SGNs jointly encode information from each place of the tonotopic map (Liberman, 1978).

Light-evoked firing rate patterns differed among the putative SGNs as stimulation rates increased (Figure 6F). There was, in general, a linear rise of discharge rates up to $100 \mathrm{~Hz}$, followed by a constant decrease as a response to mid and high stimulation rates. Nevertheless, some putative SGNs were able to maintain moderate (and to some extent synchronized) spike rates even at pulse rates of $1000 \mathrm{~Hz}$ (fibers showing computable spike probability - see Methods- at $500 \mathrm{~Hz}: 7 / 21,33.3 \%$; at $600 \mathrm{~Hz}: 3 / 18$, 16.7\%; at $700 \mathrm{~Hz}: 3 / 18,16.7 \%$; at $800 \mathrm{~Hz}: 1 / 19,5.26 \%$; at $900 \mathrm{~Hz}: 2 / 21,9.52 \%$; and at $1000 \mathrm{~Hz}$ 2/19, 10.53\%). Interestingly, the response patterns of the putative SGNs that we recorded varied across units at increasing stimulation rates (Figure EV2). We found two main types of responses; putative SGNs that rapidly adapted their spiking as stimulation rates rose (Figure EV2A), and neurons that continued spiking even at very high pulse rates (albeit with a lower frequency as compared to lower stimulation rates, Figure EV2B). 
A
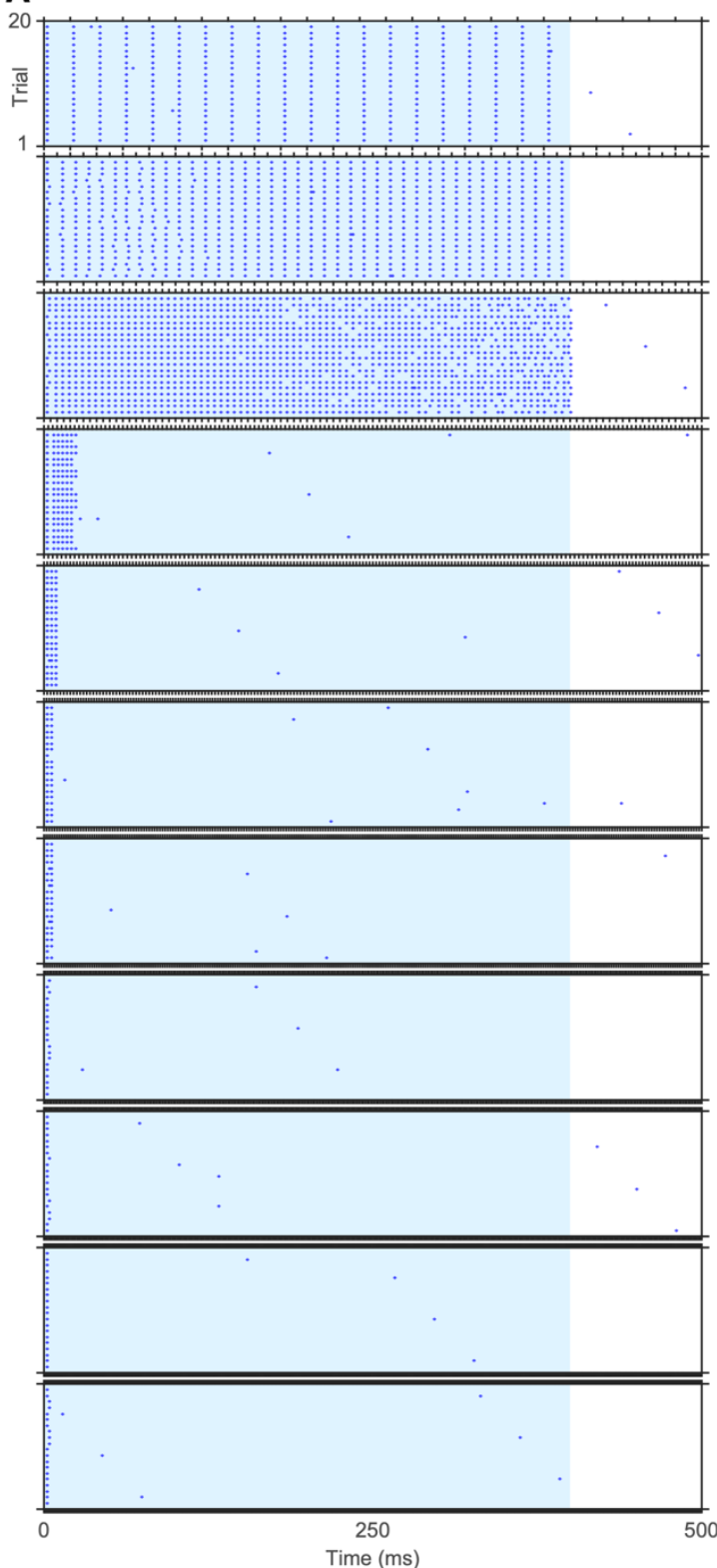

$$
\begin{gathered}
50 \mathrm{~Hz} \\
30 \mathrm{~mW} \\
1 \mathrm{~ms} \\
\\
100 \mathrm{~Hz} \\
30 \mathrm{~mW} \\
1 \mathrm{~ms} \\
\\
200 \mathrm{~Hz} \\
30 \mathrm{~mW} \\
1 \mathrm{~ms}
\end{gathered}
$$

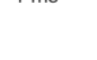

$300 \mathrm{~Hz}$

$30 \mathrm{~mW}$
$1 \mathrm{~ms}$

$1 \mathrm{~ms}$

$400 \mathrm{~Hz}$

$400 \mathrm{~mW}$
$1 \mathrm{~ms}$

$500 \mathrm{~Hz}$

$30 \mathrm{~mW}$
$1 \mathrm{~ms}$

$600 \mathrm{~Hz}$

$30 \mathrm{~mW}$
$1 \mathrm{~ms}$

$700 \mathrm{~Hz}$

$30 \mathrm{~mW}$
$0.5 \mathrm{~ms}$

$0.5 \mathrm{~ms}$

$800 \mathrm{~Hz}$

$30 \mathrm{~mW}$
$0.5 \mathrm{~ms}$

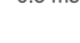

$900 \mathrm{~Hz}$

$30 \mathrm{~mW}$
$0.5 \mathrm{~ms}$

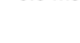

$1000 \mathrm{~Hz}$

$30 \mathrm{~mW}$
$0.5 \mathrm{~ms}$

$0.5 \mathrm{~ms}$
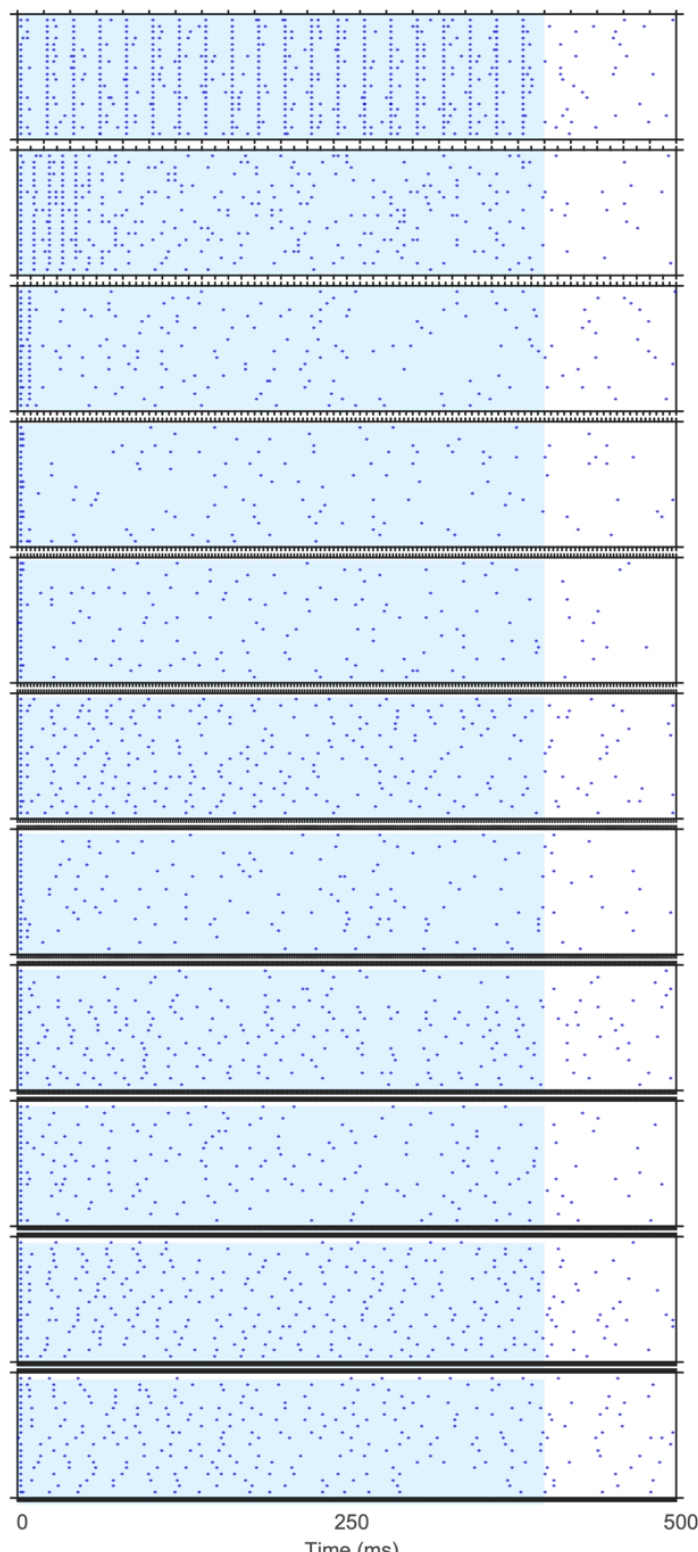

Figure EV2. Activity of two exemplary putative SGNs upon light stimulation at increasing repetition rates

(A) Raster plot showing spike timing (blue symbols) of a rapidly adapting putative SGN in response to $400 \mathrm{~ms}$ pulse trains at stimulation rates $50-1000 \mathrm{~Hz}(30 \mathrm{~mW}, 1 \mathrm{~ms}$ for $50-600 \mathrm{~Hz} ; 30 \mathrm{~mW}, 0.5 \mathrm{~ms}$ for 700-1000 Hz).

(B) Raster plot showing activity of a slowly adapting putative SGN in response to the stimulation paradigm described in (A). 


\subsection{Discussion}

Here, we characterized and optimized Chronos for its utility in fast optogenetic control of excitable cells. We demonstrate sub-millisecond off-kinetics at physiological temperature of Chronos. Using the auditory system as a fast spiking neural model circuitry, we show that Chronos supports ultrafast control of neuronal spiking. In doing so, we solved a major shortcoming that plagued optogenetics as a stimulus modality for the cochlea; the low temporal fidelity of light-driven SGN-firing, caused by the slow deactivation kinetics of the ChR2 employed so far. We found that adding sequences promoting ER-exit and plasma membrane trafficking to Chronos (Chronos-ES/TS) and using postnatal injection of the powerful AAV-PHP.B vector critically improved the in vivo utility. In conclusion, Chronos-ES/TS in combination with potent viral vectors such as AAV-PHP.B is a promising tool for auditory neuroscience and a candidate ChR for use in future optical Cls.

\subsubsection{Biophysical characterization of Chronos and improving plasma membrane expression}

Here, we compared activation and deactivation for ChR2 and Chronos and also studied the temperature dependence of gating for Chronos. In our hands Chronos deactivates about 3 times faster than ChR2 and has sub-milliseconds off-kinetics at physiological temperature with a $Q_{10}$ of 2.7. This reflects short-lived open states for Chronos resulting in lower open probability, which together with the relatively poor membrane expression likely explains the challenges we faced with using the original Chronos construct for driving SGN spiking, when using the transuterine injection in the AAV2/6-hSyn vector that we previously used successfully for the ChR2 mutant CatCh (Hernandez et al, 2014). No problems were reported in two other studies of Chronos in the auditory system using different viruses and stages of the pathway; cochlear nucleus (Hight et al, 2015) and inferior colliculus (Guo et al, 2015). A recent study using the in silico predicted ancient AAV Anc80 also achieve functional expression in the cochlea, but did not differentiate between expression in SGNs and hair cells (Duarte et al, 2018). Inspecting the Chronos-GFP expression in the cochlear nucleus (Figure 2 of (Hight et al, 2015)) and SGNs (Duarte et al, 2018) suggest a similar diffuse intracellular distribution as we observed in HEK293T cells (Figure 2) and hippocampal neurons (Figure 3) in culture as well as in SGNs in our study (Figure 4). Using these 3 cell types and vastly different conditions, we show a robust improvement of the plasma membrane expression of Chronos upon adding ER-export (Stockklausner et al, 2001; Ma et $a l, 2001$ ) and trafficking signals (Hofherr et al, 2005) of inward rectifying $\mathrm{K}^{+}$channels. Introduction of the powerful AAV-variant AAV-PHP.B (Deverman et al, 2016) was an additional factor changing the game: even the unmodified Chronos (Klapoetke et al, 2014) enabled oABRs on a regular basis. However, when comparing the functionality of Chronos-ES/TS with enhanced membrane trafficking to Chronos, transduced by the same AAV-PHP.B vector, titer, injection method and incubation time, we found increased $O A B R$ amplitudes and improved temporal fidelity of the Chronos-ES/TS-mediated 
responses, when increasing the rate of stimulation. Disentangling the contributions of the improved membrane expression and the slightly higher fraction of Chronos-positive SGNs with Chronos-ES/TS is challenging. Regardless of the precise contributions, these experiments suggest that Chronos-ES/TS will be a valuable tool for optogenetic applications requiring good temporal fidelity. Moreover, avoiding the largely intracellular localization of Chronos lowers the proteostatic stress to the cell. 


\subsubsection{Ultrafast Chronos-mediated stimulation of the auditory pathway}

Stimulation of the auditory pathway is a prime example for an application of optogenetics requiring both high speed and light sensitivity. Upon sound stimulation, SGNs fire at hundreds of $\mathrm{Hz}$ and show sub-millisecond temporal precision of spiking relative to the stimulus. When considering optogenetics for improved cochlear prosthetics, each stimulus should not exceed the energy requirements of electrical cochlear implants which are in the range of $0.2 \mu \mathrm{J}$ per pulse (Zierhofer et al, 1995). However, so far, the threshold for optogenetic activation of SGNs reported for ChR2 and ChR2 variant CatCh amounted to approximately $2 \mu \mathrm{J}$, and the responses broke down for stimulation rates exceeding $20 \mathrm{~Hz}$ (Hernandez et al, 2014).

Chronos, featuring a sub-millisecond deactivation time constant at physiological temperature (Figure 1) is an obvious candidate for cochlear optogenetics. So far, we had employed transuterine AAV2/6injections into the embryonic otocyst and observed a strong basoapical gradient of expression in the cochlea (Hernandez et al, 2014). Using AAV-PHP.B, a novel AAV vector (Deverman et al, 2016) at high titers $\left(1-2.510^{12} \mathrm{GC} / \mathrm{ml}\right)$ for injections into the cochlea of p5-p7 mice, we found highly efficient transduction of SGNs across all cochlear turns (Figure 5). The light thresholds estimated for OABRs mediated by Chronos-ES/TS in SGNs following postnatal AAV-PHP.B injections the average OABR threshold amounted to approximately $7 \mu \mathrm{J}$ (14 $\mu \mathrm{J}$ for Chronos), which is substantially higher than the energy per pulse employed for suprathreshold stimulation in eCls. Therefore, further improvements of membrane expression of Chronos or the design of fast channels with larger pore remain an important objective. At present, we can only speculate how much light will be required for an auditory percept mediated by Chronos-based cochlear optogenetics. Future studies using behavioral experiments will be required to address this point. Nonetheless, we argue that future optogenetic $\mathrm{Cls}$ might not need the high stimulation rates employed in eCls ( $800 \mathrm{~Hz}$ or higher) which might help balancing a greater energy requirement per pulse of an oCl. In most animals, oABR increased in amplitude when increasing light intensity over more than one order of magnitude (Figure 6). Hence, the output dynamic range of optical stimulation assessed as $P_{1}-N_{1}$ amplitude, on average, was $>20 d B$ compared to typically $<10 \mathrm{~dB}$ for coding with eCl (Zeng et al, 2008). This likely reflects the lower spread of excitation with optical stimulation as well as differences in the levels of Chronos-ES/TS expression among SGNs at the same tonotopic place of stimulation.

The temporal fidelity of Chronos-ES/TS-mediated optogenetic stimulation was estimated at the single SGN and the SGN population levels. The minimal OABR latency was considerably shorter $(1.01 \pm 0.09 \mathrm{~ms})$ than that of the first light-evoked potential, typically a trough, that we had previously reported for transgenic ChR2 mice (3.14 $\pm 0.26 \mathrm{~ms}$ (Hernandez et al, 2014)). Moreover, the minimal duration of the light pulse required for eliciting an $O A B R$ at high light intensity was shorter $(<100 \mu \mathrm{s})$ 
than that found before with ChR2 or CatCh ( $\geq 200 \mu \mathrm{s}$; (Hernandez et al, 2014)). Most importantly, population responses mediated by Chronos-ES/TS followed pulse rates up to at least $1000 \mathrm{~Hz}$ unlike for ChR2, where oABRs were lost below $100 \mathrm{~Hz}$. Obviously, the analysis had to be restricted to a very short measurement time window in order to track the response to such high rates and hence signal propagation along the pathway cannot be demonstrated. This ABR analysis suggests that the ChronosES/TS mediated optogenetic stimulation achieves a temporal fidelity similar to that of acoustic coding. Using juxtacellular recordings we could demonstrate firing of single SGNs in response to trains of light pulses at hundreds of $\mathrm{Hz}$ with sub-millisecond temporal precision. Such temporal fidelity of optogenetic control of SGN firing marks a major breakthrough on the way towards using optogenetic stimulation for auditory research and for the future development of the clinical optical cochlear implant. 


\subsection{Materials and Methods}

\subsubsection{Illumination during patch clamp}

Illumination at $488 \mathrm{~nm}$ was provided either by a frequency doubled solid state laser (Sapphire, Coherent) or by a diode laser (Cobolt MLD). The solid state laser was shuttered by a custom made, mechanical shutter based on the voice-coil actuator of a hard drive (Maguire et al, 2004). The diode laser provided even faster on/off times and made it possible to rapidly change amplitudes. However, its output was not as stable as the solid state laser. Especially after drastic intensity changes, the intensity oscillated by up to $10 \%$, which also led to oscillating Chronos currents. The beams of the lasers were coupled into the optical path of the microscope and focused on the sample to a spot size smaller than the field of view of the camera, to precisely determine the absolute intensity in the plane of the cells. The laser power was adjusted by neutral density filters, and the absolute power of the laser was measured at the position of the sample with a photo-diode based power-meter (PM100D, Thorlabs, Newton, NJ). A photo-diode recorded the temporal shape of the laser intensity during the experiments and digitization was performed by the patch-clamp amplifier also used for triggering the laser shutters (EPC10, HEKA, Lambrecht, Germany). The average intensity on each cell was calculated by comparing the size and exact position of the cell with the intensity profile of the laser spot. When using the mechanical shutter, artifacts lasting only two data-points were common during switching due to the strong transient currents in the voice-coil. These were removed in post processing. For the initial kinetic analysis a rapidly switchable $480 \mathrm{~nm}$ LED was placed $25 \mathrm{~mm}$ below the cells ( $5 \mathrm{~W}$ Luxeon rebel color with Lambertian dome, Philips Lumileds), that provided homogeneous whole-field illumination at a maximum of $0.27 \mathrm{~mW} / \mathrm{mm}^{2}$.

\subsubsection{Cloning}

For the cloning of pAAV_hSyn_Chronos-ES/TS as a starting material we have used pAAV-Ef1a-DIOeNpHR 3.0-EYFP (Addgene, plasmid nr. \#26966). In order to obtain flanked EYFP with ES/TS sequences we perform a classical PCR reaction. The primers that we used for this cloning were $5^{\prime}$ GAGAACCGGTCAAGAGCAGGATCAC-3' and 5'-GTGGGGTACCCCTTACACCTCGTTCTC-3'. In the second step obtained PCR fragment was digested with Agel/Acc65I (Thermo Scientific, MA, USA) (Acc65I produces compatible cohesive ends as BsrGI) gel extracted (GeneJET Gel Extraction Kit, Thermo Scientific, MA, USA) and further used for ligation. At the same time plasmid pAAV_hSyn_Chronos-GFP (Addgene, plasmid no. 59170) was also digested using restriction enzymes Agel/BsrGl and used as a backbone plasmid. All obtained ligation products were further tested by the mean of colony PCR and finally sequenced by external company. 


\subsubsection{Cell Culture and HEK-293T transfection}

HEK-293T cells (ATCC, USA) were cultured at $37^{\circ} \mathrm{C}$ and $5 \% \mathrm{CO}_{2}$ in DMEM (Gibco, USA) supplemented with $10 \%$ fetal calf serum (Gibco, Germany), and $1 \%$ penicillin/streptomycin (Sigma, Germany). One day prior to transient transfections the HEK293T cells were seeded on 24-well plates. Day after cells were transiently transfected with pAAV_hSyn_Chronos-ES/TS or pAAV_hSyn_Chronos using PEI 25.000MW (Polysciences Inc. USA). Two days after transfection cells were briefly washed and then fixed with $4 \%$ PFA for 10 min. After fixation cells were mounted with Mowiol (Sigma, Germany) and processed to confocal/STED imaging. Cells were regularly tested for mycoplasma contamination. No method of cell line authentication was used.

For characterization of gating kinetics, HEK-293T cells were transfected with 3-5 $\mu \mathrm{g}$ of plasmid DNA encoding a ChR2-YFP or a Chronos-GFP fusion protein via nucleofection using a Lonza nucleofector device and the Amaxa Cell Line Nucleofection Kit V (Lonza, Switzerland; program Q-01), following the manufacturer's instructions. The pcDNA 3.1-ChR2-YFP construct was kindly provided by Ernst Bamberg (MPI for Biophysics, Frankfurt, Germany), and Chronos-GFP was kindly provided by Edward Boyden (MIT, Cambridge, MA). After transfection, the cells were plated on poly-L-lysine coated $10 \mathrm{~mm}$ glass cover slips. Electrophysiological characterization was performed 20 to $30 \mathrm{~h}$ after transfection.

\subsubsection{Electrophysiology}

Transfection success was accessed via fluorescence in an inverted Axiovert 135 TV fluorescence microscope (ZEISS, Germany), equipped with a 40X/0.65 N.A. Achroplan objective. Only cells isolated from others and exhibiting clear membrane fluorescence signal were recorded. Whole-cell patchclamp recordings were made in voltage-clamp mode $(-60 \mathrm{mV}$ holding potential) in an EPC 10 USB amplifier (HEKA Elektronik, Germany). Current signals were low-pass filtered at $3 \mathrm{kHz}$ and digitized at 20 kHz. Patch-pipettes were prepared from PG10165-4 glass capillaries (World Precision Instruments, USA) in a PIP 6 vertical puller (HEKA Elektronik, Germany), and had resistances between 3-5 MOhm when filled with the following pipette solution (in $\mathrm{mM}$ ): $110 \mathrm{NaCl}, 10 \mathrm{Na}_{4}-\mathrm{EGTA}, 4 \mathrm{MgCl}_{2}, 10 \mathrm{HEPES}$, and 10 Glucose (pH 7.4 and osmolarity between $285-290$ mOsm). The bath solution contained (in mM): $145 \mathrm{NaCl}, 3 \mathrm{KCl}, 1 \mathrm{MgCl}_{2}, 2 \mathrm{CaCl}_{2}, 10 \mathrm{HEPES}$, and 15 Glucose (osmolarity between 310-315 mOsm and $\mathrm{pH}$ 7.35). Series resistance (always $<20 \mathrm{MOhm}$ before compensation) was electronically compensated 60 to $90 \%$. For recordings at physiological temperature, warm solution was perfused by gravitation in the recording chamber via an HPT-2 in-line heater (ALA Scientific Instruments, USA) controlled by a TC10 temperature controller (NPI Electronic, Germany). Temperature at the recording chamber was monitored via a thermistor placed in the chamber and it was maintained at $36 \pm 1{ }^{\circ} \mathrm{C}$. Light-stimulation was achieved using a $480 \mathrm{~nm}$ diode (5 W Luxeon rebel color with Lambertian dome; Philips Lumileds) controlled by a custom-built controller. Stimulation protocols consisted of $1 \mathrm{~s}$-long light steps at 
different intensities $\left(0.08,0.14,0.19,0.23,0.27 \mathrm{~mW} \mathrm{~mm}^{-2}\right)$, with a $11 \mathrm{~s}$-long dark period in between, or a $10 \mathrm{~s}$-long light chirp linearly-increasing from 0.1 to 100 (or 500 ) $\mathrm{Hz}$ and maximum amplitude of $0.27 \mathrm{~mW} \mathrm{~mm}^{-2}$ (following a $1 \mathrm{~s}$-long pre-pulse at half maximum intensity). The LED output faithfully followed the voltage command up to at least $5 \mathrm{kH}$. Light-elicited currents were analyzed offline in Matlab 2011b/2014b (Mathworks, USA), Igor Pro 6/7 (Wavemetrics, USA) and OriginPro 7 (OriginLab, USA), this included the correction of baseline drifts during the $10 \mathrm{~s}$-long chirp recordings. For recordings at $36^{\circ} \mathrm{C}$ occasionally spike-like artifacts occurred due to the in-line heater. Those spikes were removed for display purposes in Figure 1.

\subsubsection{Dissociation and culture of hippocampal neurons, AAV infection and immunocytochemistry}

E18 pregnant Wistar rats were sacrificed using $\mathrm{CO}_{2}$, embryos were removed and heads of embryos were placed in a $10 \mathrm{~cm}$ petri dish containing ice-cold dissection media (HBSS (Gibco) $+10 \mathrm{mM}$ Hepes (Gibco)). Brains were removed and collected in fresh dissection medium. Hippocampi were separated from the brain and meninges were removed. Hippocampi were digested with $2 \mathrm{ml}$ pre-warmed $37^{\circ} \mathrm{C}$ $0.05 \%$ trypsin-EDTA (Gibco, Germany) for $20 \mathrm{~min}$ at $37^{\circ} \mathrm{C}$. Trypsin was removed and the tissue was washed three times with $4^{\circ} \mathrm{C}$ dissection medium. Dissection medium was replaced with $1 \mathrm{ml}$ prewarmed NB+ (Neurobasal with 1X B-27 supplement, 1X Glutamax and Penicillin $\left(5,000 \mathrm{U} \mathrm{ml}^{-}\right.$ $\left.{ }^{1}\right) /$ Streptomycin $\left(5000 \mathrm{~g} \mathrm{ml}^{-1}\right)$; all from Gibco) and tissue was triturated by gentle pipetting. The tissue suspension was filtered through a $100 \mathrm{~mm}$ cell strainer (BD Biosciences). Cells were counted using the trypan blue exclusion method and cultured on $12 \mathrm{~mm}$ glass coverslips (Thermo Scientific) coated with poly-D-lysine (PDL, Sigma) dissolved in $0.1 \mathrm{M}$ borate buffer, in 24-well plates (CytoOne) at a density of 80,000 hippocampal neurons per $\mathrm{cm}^{2}$ in NB+ medium in a Hera Cell 240i cell culture incubator (Thermo Scientific) at $37^{\circ} \mathrm{C}$ and $5 \% \mathrm{CO}_{2}$. On DIV 10 neurons were infected with $1 \mu \mathrm{I}$ AAV2/6 Chronos, AAV2/6 Chronos-ES/TS or the same constructs packed in PHP.B virus capsid. $72 \mathrm{~h}$ after infection neurons were briefly washed with pre-warmed PBS and then fixed with $4 \%$ PFA at RT for $10 \mathrm{~min}$. After fixation and several steps of washing, neurons were subjected to immunocytochemistry (rabbit anti-Map2 primary antibody, 1:1000, Abcam and goat anti-rabbit Alexa 555 secondary antibody, 1:1000, Invitrogen) and later on to confocal microscopy (Leica SP5).

\subsubsection{Virus purification}

AAVs were generated in HEK-293T cells (ATCC) using polyethylenimine transfection (25.000 MW, Polysciences, USA) (Gray et al, 2011; Deverman et al, 2016). In brief, triple transfection of HEK-293T cells was performed using pHelper plasmid (TaKaRa/Clontech), trans-plasmid providing viral capsid PHP.B (generous gift from Ben Deverman and Viviana Gradinaru, Caltech, USA) and cis-plasmid providing Chronos or Chronos-ES/TS (Figure 1A). The cell line was regularly tested for mycoplasma. We 
harvested viral particles $72 \mathrm{~h}$ after transfection from the medium and $120 \mathrm{~h}$ after transfection from cells and the medium. Viral particles from the medium were precipitated with $40 \%$ polyethylene glycol 8000 (Acros Organics, Germany) in $500 \mathrm{mM} \mathrm{NaCl}$ for $2 \mathrm{~h}$ at $4^{\circ} \mathrm{C}$ and then after centrifugation at 4,000 $\mathrm{g}$ for $30 \mathrm{~min}$ combined with cell pellets for processing. The cell pellets were suspended in $500 \mathrm{mM}$ $\mathrm{NaCl}, 40 \mathrm{mM}$ Tris, $2.5 \mathrm{mM} \mathrm{MgCl} 2, \mathrm{pH}$ 8, and $100 \mathrm{U} \mathrm{mL}^{-1}$ of salt-activated nuclease (Arcticzymes, USA) at $37^{\circ} \mathrm{C}$ for $30 \mathrm{~min}$. Afterwards, the cell lysates were clarified by centrifugation at 2,000 $\mathrm{g}$ for $10 \mathrm{~min}$ and then purified over iodixanol (Optiprep, Axis Shield, Norway) step gradients (15\%, $25 \%, 40 \%$ and $60 \%$ ) (Zolotukhin et al, 1999; Grieger et al, 2006) at 58,400 rpm for 2.25 h. Viruses were concentrated using Amicon filters (EMD, UFC910024) and formulated in sterile phosphate-buffered saline (PBS) supplemented with $0.001 \%$ Pluronic F-68 (Gibco, Germany). Virus titers were measured using AAV titration kit (TaKaRa/Clontech) according to manufacturer's instructions by determining the number of DNase I resistant vg using qPCR (StepOne, Applied Biosystems). Purity of produced viruses was routinely checked by silver staining (Pierce, Germany) after gel electrophoresis (Novex ${ }^{\mathrm{TM}} 4-12$ \% TrisGlycine, Thermo Fisher Scientific) according to manufacturer's instruction. The presence of viral capsid proteins was positively confirmed in all virus preparations. Viral stocks were kept at $-80{ }^{\circ} \mathrm{C}$ until experimental day.

\subsubsection{Transuterine AAV injection into the cochlea}

For in vivo transduction, anesthesia was induced with a mixture of ketamine and xylazine $\left(0.125 / 5 \mathrm{mg} \mathrm{kg}^{-1}\right)$ and maintained with isoflurane (1\%-2\%). Viral inoculum ( $250 \mathrm{nl}, 4 \mathrm{e}+8$ particles $\mu \mathrm{l}^{-}$ $\left.{ }^{1}\right)$ was microinjected through the uterus into the mouse otocyst from E11.5 to E12.5 as previously described (Brigande et al, 2009; Reisinger et al, 2011). Only the left otocyst of each embryo was injected. The non-injected contralateral ear served as an internal control. AAV2/6_hSyn_Chronos-GFP and AAV2/6_hSyn_Chronos-ES/TS were purchased from the University of North Carolina Vector Core, Chapel Hill, USA.

\subsubsection{Postnatal AAV injection into the cochlea}

Postnatal AAV-injection into scala tympani of the left ear via the round window was performed at p5p7 wild-type C57BL/6 mice essentially as described (Akil et al, 2012) using AAV-PHP.B viral capsids and hSyn promoter to drive transgenic expression of opsins in SGNs. In brief, under general isoflurane anaesthesia and local analgesia achieved by means of xylocaine, the left ear was approached via a dorsal incision and the round window membrane was identified and gently punctured using a borosilicate capillary pipette that was kept in place to inject approximately 1-1.5 $\mu$ l of AAV2/6_hSynChronos $\left(2.33 \mathrm{E}+12 \mathrm{GC} \mathrm{ml}^{-1}\right)$, AAV2/6_hSyn-Chronos-ES/TS $\left(2.2 \mathrm{E}+12 \mathrm{GC} \mathrm{ml}^{-1}\right)$, PHP.B_hSyn-ChronosGFP $\left(1.07 E+12 \mathrm{GC} \mathrm{ml}^{-1}\right)$ or PHP.B_hSyn-Chronos-ES/TS-EYFP $\left(2.31 \mathrm{E}+12 \mathrm{GC} \mathrm{ml}^{-1}\right)$. After virus application, 
the tissue above the injection site was repositioned and the wound was sutured and buprenorphine (0.1 $\left.\mathrm{mg} \mathrm{kg}^{-1}\right)$ was applied as pain reliever. Recovery of the animals was then daily tracked. In all experiments, mice were randomly selected for injection. No blinding was possible since injections have to be performed in the left ear leaving the right ear as an internal control. Hence, surgery prior to stimulation needed to be done in the injected ear. Animals were then kept in a $12 \mathrm{~h}$ light/dark cycle, with access to food and water ad libitum. All experiments were done in compliance with the national animal care guidelines and were approved by the board for animal welfare of the University Medical Center Goettingen and the animal welfare office of the state of Lower Saxony (Animal protocol no. 17_1726). The calculation of animal number was performed prior to starting experiments. We planned to use the Wilcoxon Rank Sum Test and an error probability alpha smaller than 0.05, a power (1-beta) of 0.95 and effect size depending on the precise experimental protocol.

\subsubsection{Immunostaining and imaging of cochlear cryosections}

Cochleae were fixed with $4 \%$ paraformaldehyde in phosphate buffered saline $(1 \mathrm{~h})$. Sections of the cochlea were cryosectioned following 0.12 M EDTA decalcification. After incubation of sections for $1 \mathrm{~h}$ in goat serum dilution buffer ( $16 \%$ normal goat serum, $450 \mathrm{mM} \mathrm{NaCl}, 0.6 \%$ Triton X-100, $20 \mathrm{mM}$ phosphate buffer, $\mathrm{pH}$ 7.4) primary antibodies were applied over night at $4^{\circ} \mathrm{C}$. The following antibodies were used: chicken anti-GFP (catalog n.: ab13970, Abcam, 1:500), guinea pig anti-parvalbumin (catalog no.: 195004, Synaptic Systems, 1:300). Secondary AlexaFluor-labeled antibodies (goat anti-chicken 488 $\operatorname{lgG}(\mathrm{H}+\mathrm{L})$, catalog no.: A-11039, Thermo-Fisher Scientific, 1:200; goat-anti guinea pig $568 \lg \mathrm{g}(\mathrm{H}+\mathrm{L})$, catalog no. A1107, Thermo-Fisher Scientific, 1:200) were applied for $1 \mathrm{~h}$ at room temperature. Confocal images were collected using a SP5 microscope (Leica) and processed in ImageJ. Expression was considered positive when FP fluorescence in a given cell (marked by parvalbumin) was found to be higher than 3 SD above the background fluorescence of the tissue.

For FP localization analysis line profiles (length: $7.5 \mu \mathrm{m}, 7.5 \mu \mathrm{m}$ and $1.5 \mu \mathrm{m}$ for SGNs, HEK cells and hippocampal neurons, respectively, width: 3 pixels) were centered to the outer edge of the estimated cell membrane. The line profiles were oriented perpendicular to the cell edge. Sample size was 1,1 and 3 per cell for SGNs, HEK cells and hippocampal neurons, respectively. For membrane/intracellular expression ratio a maximum peak detection was performed for membranous area (defined as $-11 \mu \mathrm{m}$, $-11 \mu \mathrm{m}$ and $-0.50 .3 \mu \mathrm{m}$ for SGNs, HEK cells and hippocampal neurons, respectively) and for intracellular area (defined as $1.12 \mu \mathrm{m},-1.12 \mu \mathrm{m}$ and $0.40 .5 \mu \mathrm{m}$ for SGNs, HEK cells and hippocampal neurons, respectively). 


\subsubsection{STED microscopy}

Images were acquired using an Abberior Instruments Expert Line STED microscope, with excitation lasers at $488 \mathrm{~nm}$ and STED lasers at $595 \mathrm{~nm}, 1 \mathrm{~W}$ using a 1.4 NA 100x oil immersion objective, either in confocal or in 2D-STED mode.

\subsubsection{Optical stimulation in vivo}

The left bulla was reached using a retroauricular approach and opened to expose the cochlea. A 50/200 $\mu \mathrm{m}$ optical fiber coupled to a $594 \mathrm{~nm}$ laser (OBIS LS OPSL, $100 \mathrm{~mW}$, Coherent Inc.) was inserted into the cochlea via the round window. Irradiance was calibrated with a laser power meter (LaserCheck; Coherent Inc.).

\subsubsection{Auditory brainstem responses}

For stimulus generation and presentation, data acquisition, and off-line analysis, we used a NI System and custom-written Matlab software (The MathWorks, Inc.). Optically-evoked ABRs (oABRs) and acoustically-evoked $A B R s$ ( $a A B R s$ ) were recorded by needle electrodes underneath the pinna, on the vertex, and on the back near the legs. The difference potential between vertex and mastoid subdermal needles was amplified using a custom-designed amplifier, sampled at a rate of $50 \mathrm{kHz}$ for $20 \mathrm{~ms}$, filtered $(300-3000 \mathrm{~Hz})$ and averaged across 1000 and 500 presentations (for OABRs and aABRs, respectively). The first ABR wave was detected semi-automatically with a custom-written Matlab script in which the wave was detected for each trace in a temporal window defined by the user and for which the amplitude was bigger than the average +2 standard deviations. Thresholds were determined by visual inspection as the minimum sound or light intensity that elicited a reproducible response waveform in the recorded traces.

\subsubsection{Juxtacellular recordings from single putative SGNs}

For auditory nerve recordings, glass microelectrodes $(\sim 50 \mathrm{M} \Omega)$ were advanced through the posterior end of the anteroventral cochlear nucleus using an Inchworm micro-positioner (EXFO Burleigh, NY, USA) and aimed towards the internal auditory canal. Action potentials were amplified using an ELC03XS amplifier (NPI Electronic, Tamm, Germany), filtered $(300-3000 \mathrm{~Hz})$, digitized (National Instruments card PCle-6323), analysed and prepared for display using custom-written Matlab (The MathWorks, Inc.) software. When light-responsive fibers were found, $400 \mathrm{~ms}$-long pulse trains at repetition rates $20-1000 \mathrm{~Hz}$ were presented, leaving $100 \mathrm{~ms}$ inter-train recovery over 20 iterations for each tested rate. Different rates were tested following no particular order, being $20 \mathrm{~Hz}$ the first repetition rate presented across all units. For repetition rates higher or equal to $200 \mathrm{~Hz}$, parameters were computed if the spike probability was equal or superior to $5 \%$. If not, values were set to 0 . Phase- 
locking was quantified using the vector strength (Goldberg \& Brown, 1969), considering a cycle starting at the onset of a light pulse and ending at the onset of the subsequent pulse, and conforming to the equation: vector strength $=\frac{\sqrt{\left[?_{i=1}^{\mathrm{n}} \cos ?_{\mathrm{i}}\right]^{2}+\left[?_{\mathrm{i}=1}^{\mathrm{n}} \sin ?_{\mathrm{i}}\right]^{2}}}{\mathrm{n}}$, being $\Theta_{1}, \Theta_{2}, \ldots, \Theta_{n}$ cycle phases in which spikes occurred. The Rayleigh test was used to evaluate the significance of vector strength: if $L>13.8$, the null hypothesis is rejected at the 0.001 significance level (Hillery \& Narins, 1987) and insignificant VS were set to 0 . The spike probability was calculated as the ratio between the number of spikes and the number of light-pulses. The temporal jitter is the standard deviation of spike latency across trials. The hazard function (for the temporal jitter analysis) was calculated for each stimulation rate by simulating spiking as a Poisson process at given rates (from 10 to 1000 spikes s$^{-1}$ ).

\subsubsection{Data analysis}

The data were analysed using Matlab (Mathworks), Excel (Microsoft), Igor Pro (Wavemetrics), FIJI (ImageJ2), Origin (Microcal Software), and GraphPad Prism (GraphPad Software). Averages were expressed as mean $\pm S E M$ or mean $\pm S D$, as specified. References to data in the main text were expressed as mean \pm SEM. For statistical comparison between two groups, data sets were tested for normal distribution (the D'Agostino \& Pearson omnibus normality test or the Shapiro-Wilk test) and equality of variances (F-test) followed by two-tailed unpaired Student's t-test, or the unpaired twotailed Mann-Whitney $U$ test when data were not normally distributed and/or variance was unequal between samples.

For evaluation of multiple groups, statistical significance was calculated by using one-way ANOVA test followed by Tukey's test for normally distributed data (equality of variances tested with the BrownForsythe test) or one-way Kruskal-Wallis test followed by Dunn's test for non-normally distributed data. 


\subsection{References and notes}

Adamantidis A, Arber S, Bains JS, Bamberg E, Bonci A, Buzsáki G, Cardin JA, Costa RM, Dan Y, Goda Y, Graybiel AM, Häusser M, Hegemann P, Huguenard JR, Insel TR, Janak PH, Johnston D, Josselyn SA, Koch C, Kreitzer AC, et al (2015) Optogenetics: 10 years after ChR2 in neurons--views from the community. Nat. Neurosci. 18: 1202-1212

Akil O, Seal RP, Burke K, Wang C, Alemi A, During M, Edwards RH \& Lustig LR (2012) Restoration of Hearing in the VGLUT3 Knockout Mouse Using Virally Mediated Gene Therapy. Neuron 75: 283-293

Boyden ES, Zhang F, Bamberg E, Nagel G \& Deisseroth K (2005) Millisecond-timescale, genetically targeted optical control of neural activity. Nat Neurosci 8: 1263-1268

Brigande JV, Gubbels SP, Woessner DW, Jungwirth JJ \& Bresee CS (2009) Electroporation-mediated gene transfer to the developing mouse inner ear. Methods Mol. Biol. 493: 125-139

Busskamp V, Picaud S, Sahel JA \& Roska B (2012) Optogenetic therapy for retinitis pigmentosa. Gene Ther. 19: 169-175

Deverman BE, Pravdo PL, Simpson BP, Kumar SR, Chan KY, Banerjee A, Wu W-L, Yang B, Huber N, Pasca SP \& Gradinaru V (2016) Cre-dependent selection yields AAV variants for widespread gene transfer to the adult brain. Nat. Biotechnol. 34: 204-209

Duarte MJ, Kanumuri VV, Landegger LD, Tarabichi O, Sinha S, Meng X, Hight AE, Kozin ED, Stankovic KM, Brown MC \& Lee DJ (2018) Ancestral Adeno-Associated Virus Vector Delivery of Opsins to Spiral Ganglion Neurons: Implications for Optogenetic Cochlear Implants. Mol. Ther. Available at: http://www.sciencedirect.com/science/article/pii/S1525001618302569 [Accessed July 31, 2018]

Goldberg JM \& Brown PB (1969) Response of binaural neurons of dog superior olivary complex to dichotic tonal stimuli: some physiological mechanisms of sound localization. J. Neurophysiol. 32: 613636

Gradinaru V, Zhang F, Ramakrishnan C, Mattis J, Prakash R, Diester I, Goshen I, Thompson KR \& Deisseroth K (2010) Molecular and Cellular Approaches for Diversifying and Extending Optogenetics. Cell 141: 154-165

Gray SJ, Choi VW, Asokan A, Haberman RA, McCown TJ \& Samulski RJ (2011) Production of Recombinant Adeno-Associated Viral Vectors and Use in In Vitro and In Vivo Administration. In Current Protocols in Neuroscience, Crawley JN Gerfen CR Rogawski MA Sibley DR Skolnick P \& Wray S (eds) Hoboken, NJ, USA: John Wiley \& Sons, Inc. Available at: http://doi.wiley.com/10.1002/0471142301.ns0417s57 [Accessed January 19, 2018]

Grieger JC, Choi VW \& Samulski RJ (2006) Production and characterization of adeno-associated viral vectors. Nat. Protoc. 1: 1412-1428

Guo, W., Hight, A.E., Chen, J.X., Klapoetke, N.C., Hancock, K.E., Shinn-Cunningham, B.G., Boyden, E.S., Lee, D.J., and Polley, D.B. (2015). Hearing the light: neural and perceptual encoding of optogenetic stimulation in the central auditory pathway. Scientific Reports 5.

Hernandez VH, Gehrt A, Reuter K, Jing Z, Jeschke M, Mendoza Schulz A, Hoch G, Bartels M, Vogt G, Garnham CW, Yawo H, Fukazawa Y, Augustine GJ, Bamberg E, Kügler S, Salditt T, de Hoz L, Strenzke N \& Moser T (2014) Optogenetic stimulation of the auditory pathway. J. Clin. Invest. 124: 1114-1129 
Hight AE, Kozin ED, Darrow K, Lehmann A, Boyden E, Brown MC \& Lee DJ (2015) Superior temporal resolution of Chronos versus channelrhodopsin-2 in an optogenetic model of the auditory brainstem implant. Hear. Res. 322: 235-241

Hillery CM \& Narins PM (1987) Frequency and time domain comparison of low-frequency auditory fiber responses in two anuran amphibians. Hear. Res. 25: 233-248

Hofherr A (2005) Selective Golgi export of Kir2.1 controls the stoichiometry of functional Kir2.x channel heteromers. J. Cell Sci. 118: 1935-1943

Hofherr A, Fakler B \& Klöcker N (2005) Selective Golgi export of Kir2.1 controls the stoichiometry of functional Kir2.x channel heteromers. J. Cell Sci. 118: 1935-1943

Jeschke M \& Moser T (2015) Considering optogenetic stimulation for cochlear implants. Hear. Res. 322: 224-234

Kim CK, Adhikari A \& Deisseroth K (2017) Integration of optogenetics with complementary methodologies in systems neuroscience. Nat. Rev. Neurosci. 18: 222-235

Klapoetke NC, Murata Y, Kim SS, Pulver SR, Birdsey-Benson A, Cho YK, Morimoto TK, Chuong AS, Carpenter EJ, Tian Z, Wang J, Xie Y, Yan Z, Zhang Y, Chow BY, Surek B, Melkonian M, Jayaraman V, Constantine-Paton M, Wong GK-S, et al (2014) Independent optical excitation of distinct neural populations. Nat. Methods 11: 338-346

Kral A, Hartmann R, Mortazavi D \& Klinke R (1998) Spatial resolution of cochlear implants: the electrical field and excitation of auditory afferents. Hear. Res. 121: 11-28

Lalwani AK, Walsh BJ, Reilly PG, Muzyczka N \& Mhatre AN (1996) Development of in vivo gene therapy for hearing disorders: introduction of adeno-associated virus into the cochlea of the guinea pig. Gene Ther. 3: 588-592

Lenarz T (2018) Cochlear implant - state of the art. GMS Curr. Top. Otorhinolaryngol. Head Neck Surg. 16: Available at: https://www.ncbi.nlm.nih.gov/pmc/articles/PMC5818683/ [Accessed July 31, 2018]

Liberman MC (1978) Auditory-nerve response from cats raised in a low-noise chamber. J. Acoust. Soc. Am. 63: 442-455

Ma D, Zerangue N, Lin Y-F, Collins A, Yu M, Jan YN \& Jan LY (2001) Role of ER Export Signals in Controlling Surface Potassium Channel Numbers. Science 291: 316-319

Mager T, Morena DL de la, Senn V, Schlotte J, D'Errico A, Feldbauer K, Wrobel C, Jung S, Bodensiek K, Rankovic V, Browne L, Huet A, Jüttner J, Wood PG, Letzkus JJ, Moser T \& Bamberg E (2018) High frequency neural spiking and auditory signaling by ultrafast red-shifted optogenetics. Nat. Commun. 9: 1750

Maguire LP, Szilagyi S \& Scholten RE (2004) High performance laser shutter using a hard disk drive voice-coil actuator. Rev. Sci. Instrum. 75: 3077

Mardinly AR, Oldenburg IA, Pégard NC, Sridharan S, Lyall EH, Chesnov K, Brohawn SG, Waller L \& Adesnik H (2018) Precise multimodal optical control of neural ensemble activity. Nat. Neurosci. 21: 881-893

Nagel G, Ollig D, Fuhrmann M, Kateriya S, Musti AM, Bamberg E \& Hegemann P (2002) Channelrhodopsin-1: a light-gated proton channel in green algae. Science 296: 2395-2398 
Nagel G, Szellas T, Huhn W, Kateriya S, Adeishvili N, Berthold P, Ollig D, Hegemann P \& Bamberg E (2003) Channelrhodopsin-2, a directly light-gated cation-selective membrane channel. Proc. Natl. Acad. Sci. 100: 13940-13945

Reisinger E, Bresee C, Neef J, Nair R, Reuter K, Bulankina A, Nouvian R, Koch M, Buckers J, Kastrup L, Roux I, Petit C, Hell SW, Brose N, Rhee J-S, Kugler S, Brigande JV \& Moser T (2011) Probing the Functional Equivalence of Otoferlin and Synaptotagmin 1 in Exocytosis. J. Neurosci. 31: 4886-4895

Richter C-P, Rajguru SM, Matic Al, Moreno EL, Fishman AJ, Robinson AM, Suh E \& Walsh JT (2011) Spread of cochlear excitation during stimulation with pulsed infrared radiation: inferior colliculus measurements. J. Neural Eng. 8: 056006

RST-001 Phase I/II Trial for Advanced Retinitis Pigmentosa - Full Text View - ClinicalTrials.gov Available at: https://clinicaltrials.gov/ct2/show/NCT02556736 [Accessed February 20, 2018]

Sahel J-A \& Roska B (2013) Gene therapy for blindness. Annu. Rev. Neurosci. 36: 467-488

Stockklausner C, Ludwig J, Ruppersberg JP \& Klöcker N (2001) A sequence motif responsible for ER export and surface expression of Kir2.0 inward rectifier K(+) channels. FEBS Lett. 493: 129-133

WHO (2006) Primary Ear \& Hearing Care Training Resource World Health Organization

Wrobel C, Dieter A, Huet A, Keppeler D, Duque-Afonso CJ, VogI C, Hoch G, Jeschke M \& Moser T (2018) Optogenetic stimulation of cochlear neurons activates the auditory pathway and restores auditorydriven behavior in deaf adult gerbils. Sci. Transl. Med. 10: eaao0540

Zeng FG (2017) Challenges in Improving Cochlear Implant Performance and Accessibility. IEEE Trans. Biomed. Eng. 64: 1662-1664

Zeng F-G, Rebscher S, Harrison WV, Sun X \& Feng H (2008) Cochlear Implants:System Design, Integration and Evaluation. IEEE Rev. Biomed. Eng. 1: 115-142

Zierhofer CM, Hochmair-Desoyer IJ \& Hochmair ES (1995) Electronic design of a cochlear implant for multichannel high-rate pulsatile stimulation strategies. IEEE Trans. Rehabil. Eng. 3: 112-116

Zolotukhin S, Byrne BJ, Mason E, Zolotukhin I, Potter M, Chesnut K, Summerford C, Samulski RJ \& Muzyczka N (1999) Recombinant adeno-associated virus purification using novel methods improves infectious titer and yield. Gene Ther. 6: 973-985 


\subsection{Acknowledgments}

We would like to thank Dr. Edward Boyden for providing the Chronos-EGFP construct and Rachel Care for contributing in the initial phase of the project. We thank Andrea Koch, Daniela Gerke, Sandra Gerke and Christiane Senger-Freitag for expert technical assistance and Gerhard Hoch for devising hard and software for system physiology and Jakob Neef for the help with STED microscopy. We thank Camin Dean for providing hippocampal neuronal culture. We thank Blanche Schwappach for feedback on the manuscript.

Funding: This work was funded by the European Research Council through the Advanced Grant 'OptoHear" to T.M. under the European Union's Horizon 2020 Research and Innovation program (grant agreement No. 670759) and was further supported by the German Ministry of Research and Education through the Bernstein Center for Computational Neuroscience to A.N., F.W. and T.M. (under grant number 01GQ1005A) and the grant Optical Cl (under grant number 13N13729) to T.M.) and the German Research Foundation (through the DFG-Research Center (FZT103) and Cluster of Excellence (EXC171) Center for Nanoscale Microscopy and Molecular Physiology of the Brain to T.M., as well as the Leibniz Program to T.M.) and was further supported by grants from the German Research Foundation through the Collaborative Research Center 889 (project research rotation of A.G., C.W., T.D.).

Competing interests: The authors declare no competing interests. 


\section{Multiscale, multimodal imaging of the native and implanted cochlea}

\section{Authors:}

Daniel Keppeler ${ }^{1,2,}{ }^{*}$, Carlos J. Duque-Afonso ${ }^{1,2}$, Mareike Töpperwien ${ }^{3,4}$, Alexander Meyer ${ }^{5}$, Tobias Moser ${ }^{1,2,4,6^{*}}$

\section{Affiliations:}

${ }^{1}$ Institute for Auditory Neuroscience and InnerEarLab, University Medical Center Göttingen, Germany. ${ }^{2}$ Göttingen Graduate School for Neurosciences and Molecular Biosciences, University of Göttingen, Germany.

${ }^{3}$ Institute for X-Ray Physics, University of Göttingen, Germany.

${ }^{4}$ Center for Nanoscopy and Molecular Physiology of the Brain, Göttingen, Germany.

${ }^{5}$ InnerEarLab, Department of Otolaryngology, University Medical Center Göttingen, Germany.

${ }^{6}$ Auditory Neuroscience and Optogenetics laboratory, German Primate Center, Göttingen, Germany.

*To whom correspondence should be addressed:

Daniel Keppeler, Email: daniel.keppeler@med.uni-goettingen.de

Dr. Tobias Moser, Email: tmoser@gwdg.de 


\section{Author contributions}

D.K. and T.M. designed the study. D.K. performed implantation in rodent cochleae. D.K. performed segmentation and analysis of CT data. C.J.D.-A. performed light simulations. M.T. improved CT setup and implemented phase-reconstruction algorithm. A.M. performed implantation of cochlear implants in marmosets. D.K. and T.M. took a lead on preparing the manuscript.

Detailed author contributions of D. Keppeler (D.K.):

- Experimental work

- $\mathrm{Cl}$ implantation in rodents

- Sample preparation

○ X-ray tomographic imaging (together with M.T.)

- Segmentation of image stacks

○ Figure S2: Images were published in my master thesis.

- Analysis

- 3D quantification of segmented data

- Frequency-place estimations with Greenwood function

○ Statistics

- Preparation of the manuscript (together with T.M. and other co-authors)

C.J.D.-A. contributions were removed from this thesis, but will be part of the publication. 


\subsection{Abstract}

The cochlea, harboring the hearing end organ of Corti, is an intricate structure hidden in the temporal bone. Compared to other sensory organs such as the eye, the cochlea has remained poorly accessible for investigation, for example, by imaging. This limitation also concerns the further development of technology for restoring hearing in case of cochlear dysfunction, which requires quantitative information on spatial dimensions and neural status of the cochlea. Here, we employed phase-contrast X-ray tomography and light sheet microscopy for multiscale imaging of cochlear morphology in species that serve as established animal models for auditory research. We provide a systematic reference for morphological parameters relevant for cochlear implant development for rodent and non-human primate models. Based on imaging inserted electrical and optical cochlear implants we derive a baseline for functional studies on hearing restoration. We simulate the spread of light from the emitters of the optical implants within the reconstructed cochlea, which indicates a spatially narrow excitation of opsin-expressing spiral ganglion neurons. Finally, we devised an automated approach for estimating the fraction of transduced spiral ganglion neurons based on light-sheet microscopy of the cleared cochlea.

\subsection{Significance Statement}

For developing the optical cochlear implant (oCl), knowledge of the cochlear morphology is crucial. Here, we employed multiscale X-ray and fluorescence imaging for quantitative assessment of scala tympani, scala vestibule et media and Rosenthal's canal for species commonly used in auditory research. Further, we demonstrate feasibility of oCl implantation for rodents and the marmoset, as a non-human primate model. Using modeling of light propagation from the emitters of the oCl we estimate the frequency selectivity and range covered by optical stimulation in the cochlea. 


\subsection{Introduction}

In case of profound sensorineural hearing impairment, cochlear implants ( $\mathrm{Cls}$ ) partially restore hearing by providing auditory information to the brain. Electrical stimulation of the spiral ganglion neurons (SGNs) enables speech understanding in the majority of the approximately 500,000 users worldwide. However, current clinical Cls are limited from their wide current spread (Kral et al., 1998) resulting in a limited coding of spectral information (Kang et al., 2009). Recently, cochlear optogenetics was proposed to use light to drive the auditory nerve (Duarte et al., 2018; Hernandez et al., 2014; Mager et al., 2018; Wrobel et al., 2018). As light can be better confined in space, future optical Cls (oCls) with lower spread of excitation in the cochlea promise improved speech comprehension in noisy background as well as greater music appreciation (Dombrowski et al., 2018; Jeschke and Moser, 2015). For oCls to be developed towards a medical device, currently major efforts are undertaken to devise multichannel optical stimulators for the cochlea (Ayub et al., 2016; Goßler et al., 2014; Klein et al., 2018; Schwaerzle et al., 2016). As is the case for the electrodes of current Cls, future oCls will place multiple stimulation channels, here microscale emitters, along the tonotopic axis of the cochlea. Further development of the oCls requires precise estimates of parameters such as scala tympani size, bending radius, appropriate encapsulation and probe stiffness. Moreover, cochlear optogenetics employs gene transfer to the SGNs for which adeno-associated viruses (AAVs) seem promising candidate vectors (Hernandez et al., 2014; Mager et al., 2018; Wrobel et al., 2018). AAV delivery has used injection of virus suspension via the round window (Mager et al., 2018) or directly into the Rosenthal's canal (Wrobel et al., 2018). Estimation of the required virus load for injection into the cochlea relies on understanding the volumetric proportions of the inner ear. Therefore, the volumes of Rosenthal's canal and the scalae tympani, vestibuli et media needed to be evaluated. Finally, the efficiency and safety of viral gene transfer requires the quantification of SGN density and transduction rate.

Employing multiscale X-ray and fluorescence imaging, here we provide a comprehensive analysis of cochlear morphology for mouse, rat, gerbil, guinea pig and marmoset as animal models for auditory research. Each of these animal models offers unique advantages. The mouse is readily available for optogenetic manipulation (e.g. (Zeng and Madisen, 2012). Channelrhodopsin-expressing transgenic lines are available also for rats (Tomita et al., 2009; Witten et al., 2011) that offer a larger cochlea and can carry larger weight implants then mice (Irving et al., 2013; King et al., 2016; Lu et al., 2005). Gerbils and guinea pigs are established rodent models for auditory research and offer a larger cochlea. Gerbils, which have low frequency hearing more similar to human, have already been employed for cochlear optogenetics (Wrobel et al., 2018). Finally, we focus our analysis on the marmoset, as a well established non-human primate model for auditory research (e.g. (Johnson et al., 2012; Osmanski and 
Wang, 2011). Marmosets possess a rich vocalization, are highly communicative and a pitch perception mechanism to humans is shared (Song et al., 2016). Therefore, we compare state-of-the-art eCl to newly designed oCls in marmoset cochleae and estimate the optical spread of excitation using MonteCarlo ray tracing simulation.

\subsection{Results}

\subsubsection{Multiscale, multimodal photonic imaging of cochlear morphology}

We combined X-ray tomography and lightsheet microscopy for multiscale, multimodal photonic imaging of cochlear morphology. Using phase contrast $X$-ray tomography (Figure $1 \mathrm{~A}$ ) enabled us to resolved cochlear soft tissue, whereby we focused on the spiral ganglion and the basilar membrane (Figure 1B, C). Our custom X-ray tomography setup allowed multiscale studies of the cochlear anatomy down to a voxel size of $2 \mu \mathrm{m}$. An example of a raw volume rendering of a guinea pig cochlea dataset is shown in Figure 1C. The hemisected cochlear reconstruction reveals the scalae tympani and media et vestibule, separated by the basilar membrane as well as the Rosenthal canal. The same set-up enables the analysis of differently sized cochleae from various species: from mouse to marmosets (Figure 1D). The species differ in shape, number of turns, overall size of the cochlea and position of the round and oval window can be appreciated in Figure 1D. Human data were reconstructed from published microCT data. Quantification of the cochlear morphology as required e.g. design of appropriately shaped and sized o/eCls as well as for modeling of SGN-stimulation via $\mathrm{Cl}$ is presented below.

Lightsheet microscopy was performed by Carlos Duque Afonso and the results are not included in this thesis. 

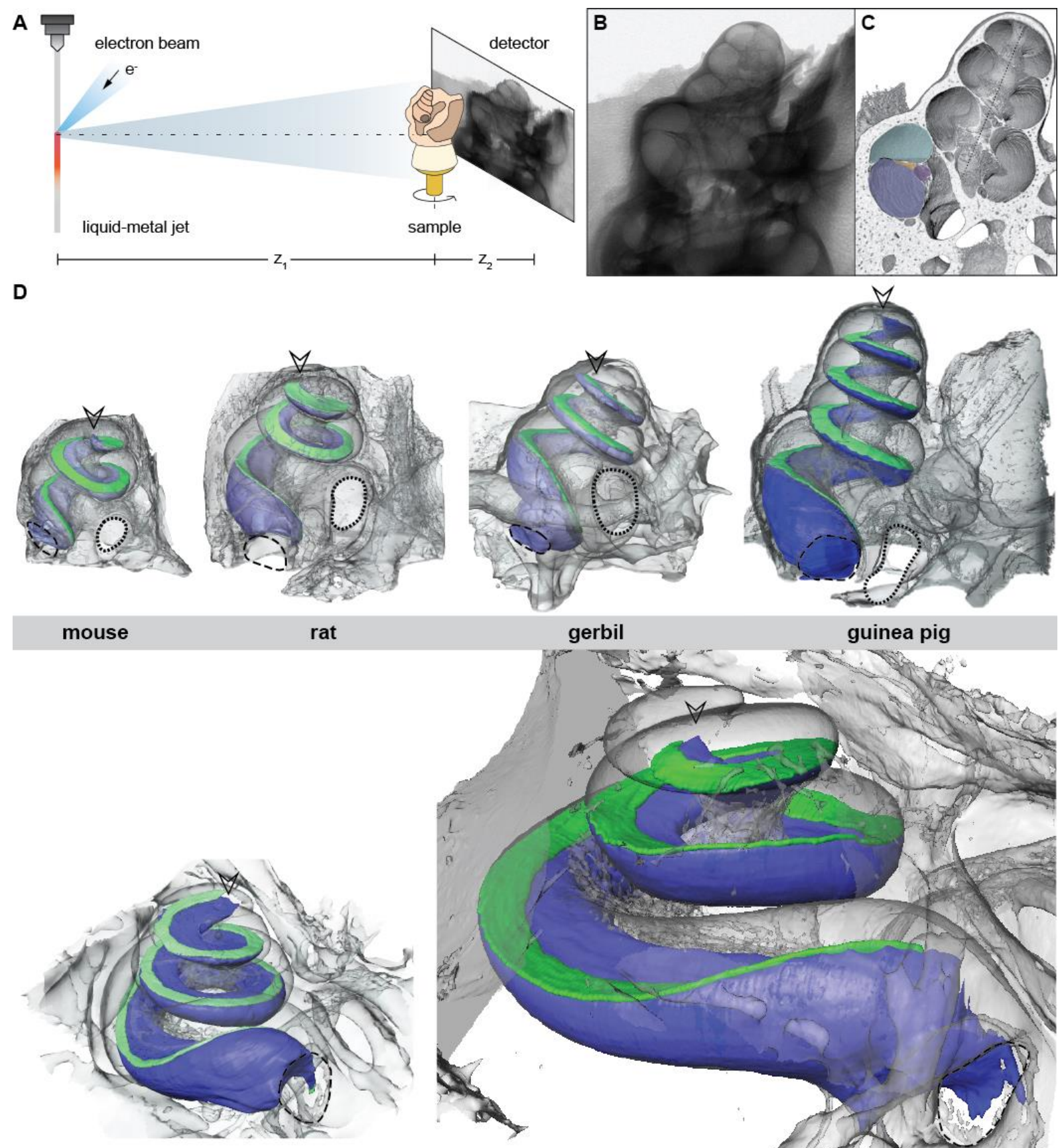

marmoset

gerbil

guinea pig
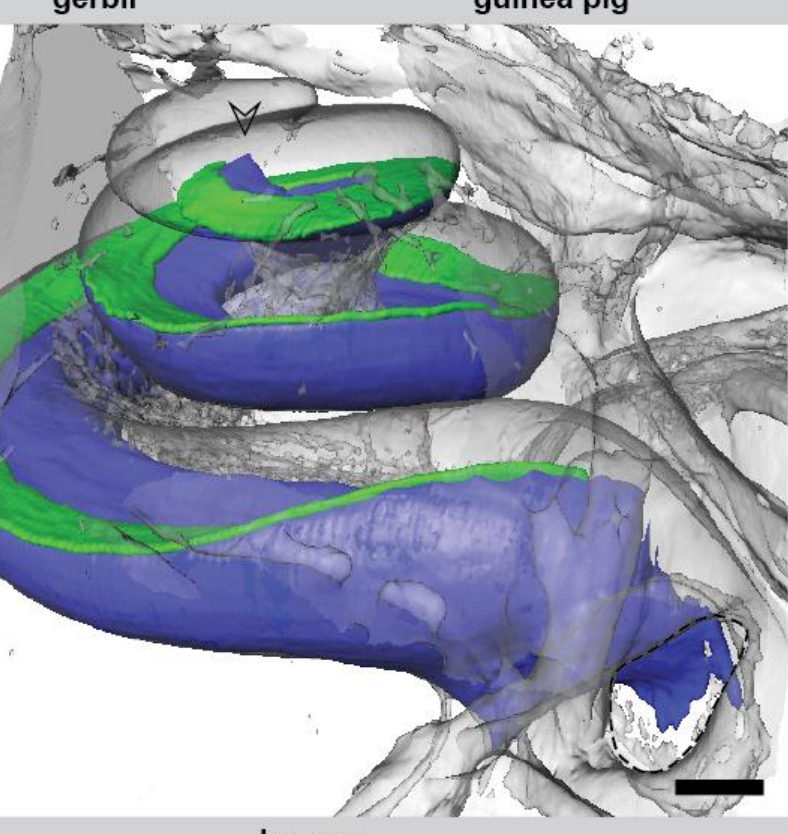

\section{Figure 1. 3D models of rodent and primate cochleae}

(A) Experimental setup. X-ray beam is generated by a liquid-metal jet microfocus tube and are detected by a high-resolution scintillator-based CCD camera.

(B) Raw 2D projection before wave propagation and phase retrieval algorithm.

(C) Volume rendering of reconstructed image stack. Scala tympani (blue), scala vestibuli et media green), Rosenthal's canal (purple), osseous spiral lamina (orange), basilar membrane (black line) and modiolar axis (dashed line).

(D) Segmented cochleae visualized with bone (gray), basilar membrane (green) scala tympani (blue), round window (dashed line), oval window (dotted line) and apex (arrow). Scale bar $1 \mathrm{~mm}$. 
A
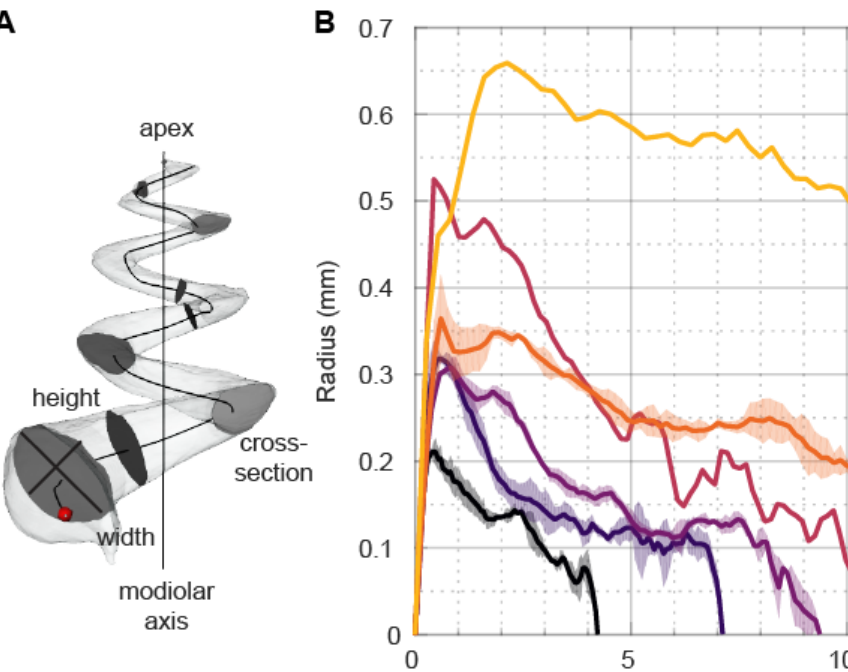

Distance from cochlear base $(\mathrm{mm})$
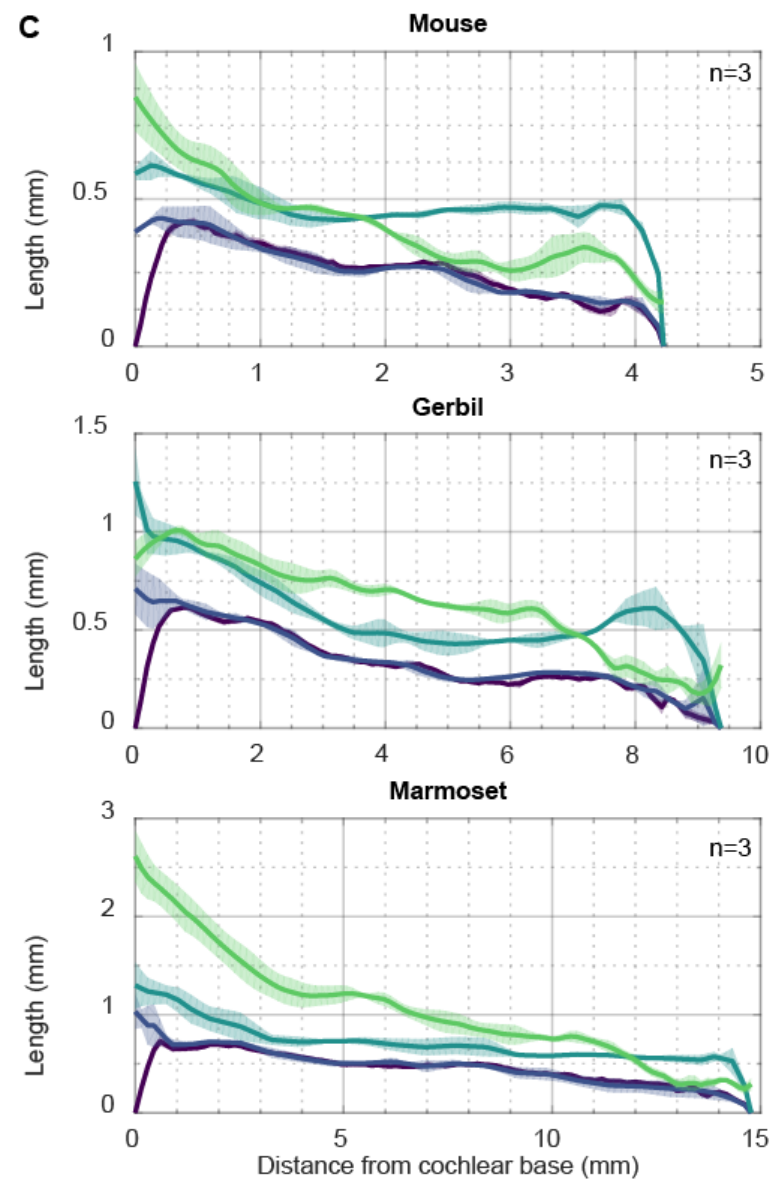
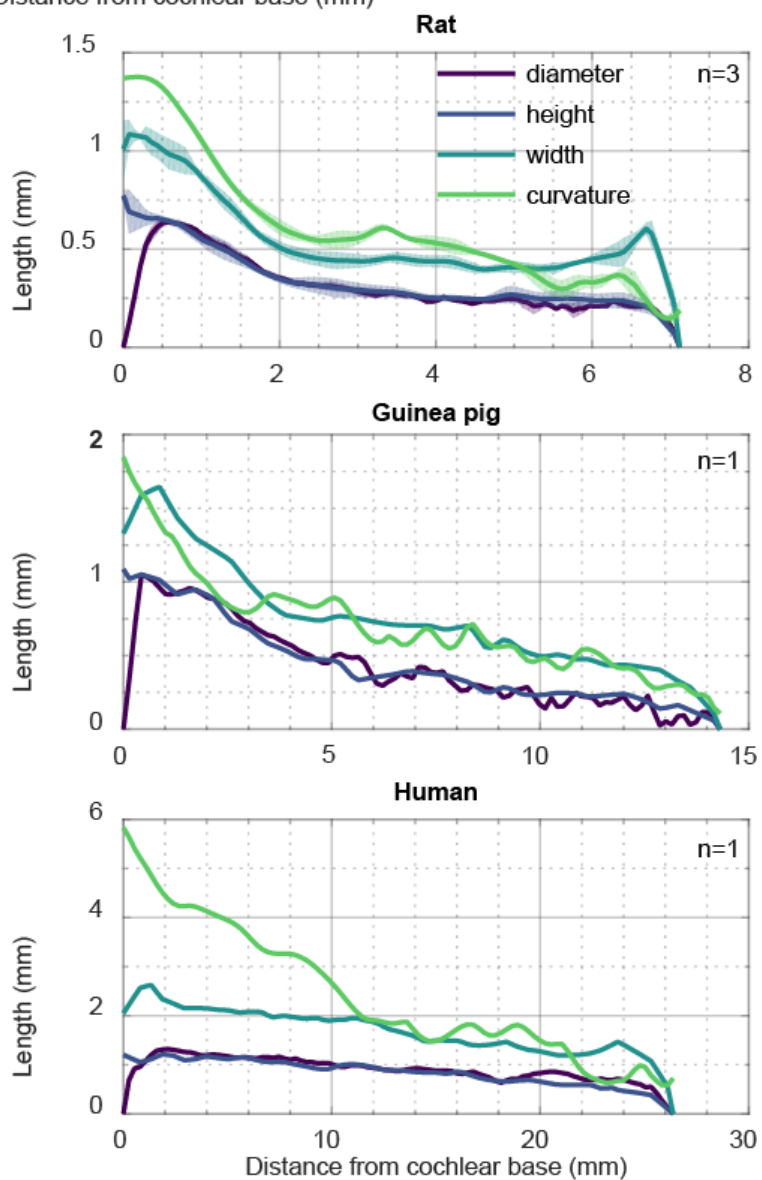

Figure 2. Measurements of the scala tympani crucial for cochlear implantation

(A) Segmented scala tympani with smoothed centerline beginning at the round window (red dot). Centerline was used as trajectory for virtual cross-sections (gray).

(B) Smallest radius of the scala tympani from base to apex. Data were scaled along the distance dimension to fit the mean length of the scala tympani. The shaded region indicates the standard deviation of the mean, $n$ as in (C).

(C) Measurements of parameter crucial for cochlear implant design considerations. Diameter is derived the centerline calculation, height and width were determined in virtual sections, curvature represents the radius from the modiolar axis to the centerline of scala tympani. $\mathrm{N}$ indicated animal count. Please note that the different species are plotted in different scales. 


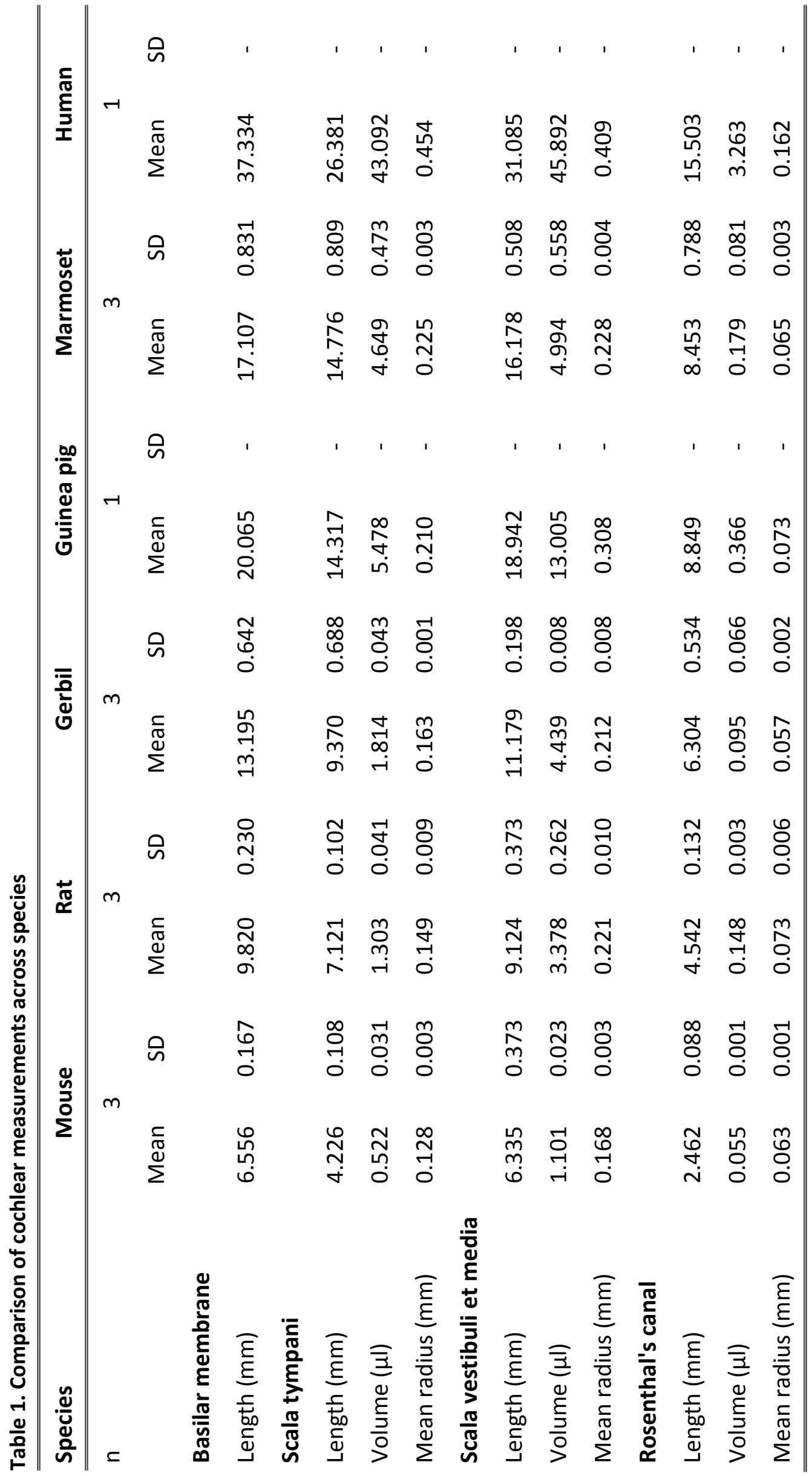




\subsubsection{Quantifying scala tympani for cochlear implant design and insertion}

For considerations on $\mathrm{Cl}$ design and for modeling of SGN-stimulation via $\mathrm{Cl}$ measurements of the scala tympani are most crucial, as this is where the $\mathrm{Cl}$ electrodes are placed. Here, we quantified $\mathrm{X}$-ray tomography data. Following 3-dimensional reconstruction (Figure 1), we calculated the centroid for virtual sections of scala tympani along the turns by determining the minimum radius by means of the closest point on the cavity's surface for each sampling point (Figure 2A). This resulted in a centerline for scala tympani (Figure 2A). For all analyzed species, the minimum radius was largest at the cochlear base close to the round window and smallest at the apex (Figure 2B). Assuming an $\mathrm{Cl}$ covering $60 \%$ of scala tympani length (Lee et al., 2010), the front of the $\mathrm{Cl}$ should be limited to a radius of $0.13 \mathrm{~mm} / 0.12 \mathrm{~mm} / 0.12 \mathrm{~mm} / 0.15 \mathrm{~mm} / 0.23 \mathrm{~mm} / 0.44 \mathrm{~mm}$ in mice, rats, gerbils, guinea pigs, marmosets and humans, respectively (Figure 2B, see also Table 1). The automatically determined diameter was identical with the height resulting from semi-automatic measurements on virtual sections, which indicates accuracy of the automatic analysis.

The bending radius, here referred to as radius of curvature (short curvature), has great relevance for the design of waveguide-based and light-emitting diode (LED) oCls. LEDs such as blue GaN-LEDs (Laubsch et al., 2010) are stiff, and hence their size and pitch within the array on a flexible substrate (Goßler et al., 2014) need to accommodate the curvature of the coiled scala tympani. To be able to cover $60 \%$ of the cochlear length in mice a radius of curvature of $0.29 \mathrm{~mm}$ has to be obeyed. In contrast, the cochleae rats, marmosets or humans provide greater radii of curvature: $0.51 \mathrm{~mm}$, $0.80 \mathrm{~mm}$ and $1.64 \mathrm{~mm}$. Regardless, all radii of curvature require the use of microscale LEDs ( $\mu$ LEDs) for the sake of optoelectronic (active) oCls. Considering such $\mu$ LED-based oCls that feature a mostly twodimensional shape due to their circuit plane, an oval-shaped design of the oCl could make use of the about 1.9 times larger width compared to the height of the tympanic duct.

\subsubsection{Insertion studies for $\mathrm{OCl}$ and $\mathrm{eCl}$ in different species}

When starting the insertion studies, we first tested various combinations of carrier materials and encapsulations in explanted mouse cochleae. Among the first attempts of an oCl insertion we used a plain polyimide substrate of $15 \mu \mathrm{m}$ thickness without encapsulation. It could be implanted into the mouse cochlea up to its full length of $5 \mathrm{~mm}$, but in subsequent X-ray tomography was shown to cut through the basilar membrane (Figure 3A inset). Hence, we thereafter encapsulated the probes. We first turned to dipping into silicone and thereafter spinning the probes, which resulted in a cylindrical shape of the polyimide-based probes and mechanical properties that were very well suitable for implantation (Figure S2). 
We then turned to encapsulating $\mu$ LED arrays following the design published earlier (Goßler et al., 2014). Those encapsulated $\mu$ LED arrays could be inserted into scala tympani via the round window for approximately $4.7 \mathrm{~mm}$ without detectable damage to the cochlea (Figure $3 \mathrm{~A}$, see also Table 2). When approximating the frequency-place map of Greenwood (Greenwood, 1990), the insertion covered a frequency range from $2.572 .2 \mathrm{kHz}$ of a total hearing range from $0.679 .3 \mathrm{kHz}$ which corresponds to 4.9 out of 7.0 octaves. The $93 \mu \mathrm{LEDs}$ accommodated on the $4.6 \mathrm{~mm}$ intracochlear portion would correspond to a dense coverage of approximately 20 channels per octave. Further, $\mu$ LED orientation appeared optimal: the active $\mu$ LED surface was facing Rosenthal's canal.

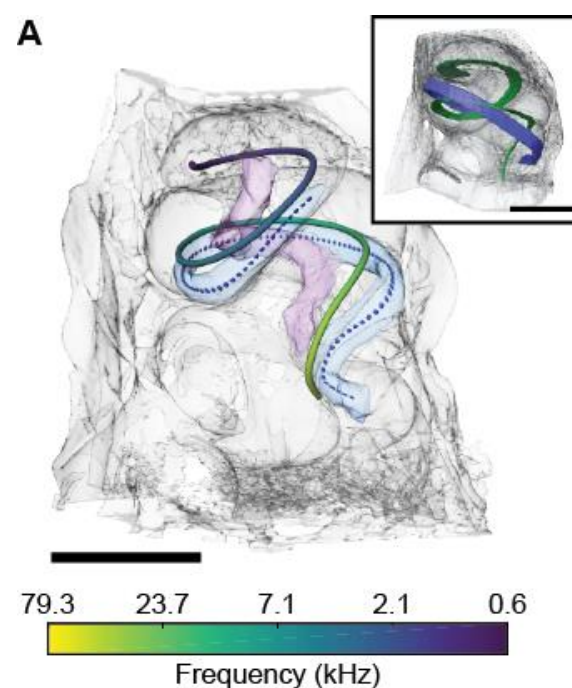

A

Figure 3. Implantation trials in rodent cochleae

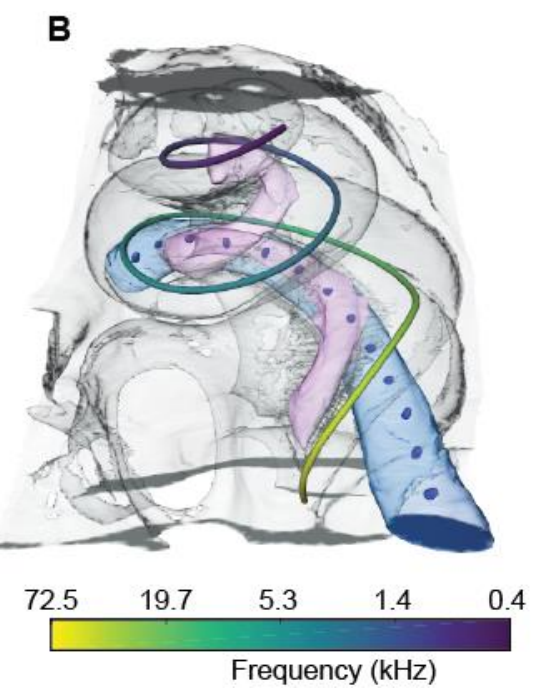

(A) Mouse cochlea with an optical cochlear implant comprising $93 \mu$ LEDs covering a frequency range from $72.2-2.5 \mathrm{kHz}$. Insertion depth: $4.6 \mathrm{~mm}$. Basilar membrane is color-coded for corresponding frequency, Rosenthal's canal (purple), LED (blue), silicone (light blue) and bone (gray). View from lateral. Inset shows a mouse cochlea with a probe penetrating the basilar membrane (green) at 0.5 turns. In this case the polyimide substrate (blue) was not encapsulated in silicone. View from medial. Scale bar: $1 \mathrm{~mm}$.

(B) Rat cochlea with an optical cochlear implant comprising $13 \mu$ LEDs covering a frequency range from 49.4-6.7 kHz. Insertion depth: $3.6 \mathrm{~mm}$. View from lateral.

We next turned to species with larger cochleae for investigating the utility of mold encapsulation. Using a state-of-the art mold-injection process, a low-density $\mu$ LED array (13 $\mu$ LEDs, pitch of $300 \mu \mathrm{m})$ was encapsulated yielding an outer diameter of $340 \mu \mathrm{m}$. The probe was inserted into scala tympani of a rat cochlea in situ. This way we achieved an implantation depth of $3.6 \mathrm{~mm}$ (Figure 3B). Despite the round profile orientation of the probe, $\mu$ LEDs were well positioned in regard to the Rosenthal's canal. However, the insertion depth was much inferior to what we found above with probes coated by dipping and spinning in the smaller cochlea of the mouse (Figure $3 \mathrm{~A}$ ).

Finally, we performed a comparative insertion study of eCls and LED-based oCls in the marmoset, a non-human primate model. Using cadaver skulls, we performed a posterior tympanotomy and inserted the Cls via the round window. The probes were specially designed to fit the marmoset cochlea: eCls 
had a diameter of $300 \mu \mathrm{m}$ with 10 electrodes and an electrode spacing of $1000 \mu \mathrm{m}$ and $1500 \mu \mathrm{m} . \mathrm{oCls}$ were designed to closely resemble the eCls with 10 LEDs $(250 \times 200 \mu \mathrm{m})$ and an LED spacing of $500 \mu \mathrm{m}$. They were dip-coated in silicone with a final diameter of approximately $260 \mu \mathrm{m}$. eCl and oCl could be implanted for $8.9 \mathrm{~mm} / 7.6 \mathrm{~mm}$ (Figure 4) covering $60 \% / 51 \%$ of the scala tympani, respectively. In case of the oCl 10 LEDs were placed on $4.5 \mathrm{~mm}$ with a mean distance of $671 \pm 93 \mu \mathrm{m}$ to the centerline of Rosenthal's canal. For eCls 6 and 7 electrode contacts were located in the scala tympani with a mean distance of $674 \pm 24 \mu \mathrm{m}$.

In regard of volumetric proportions within the cochlea, we found Rosenthal's canal's volume to be $0.055 \mu \mathrm{L} / 0.148 \mu \mathrm{L} / 0.095 \mu \mathrm{L} / 0.366 \mu \mathrm{L} / 0.179 \mu \mathrm{L} / 3.263 \mu \mathrm{L}$ in mouse, rat, gerbil, guinea pig, marmoset and human, respectively, in accordance with literature for mouse and guinea pig (Table S1).

A
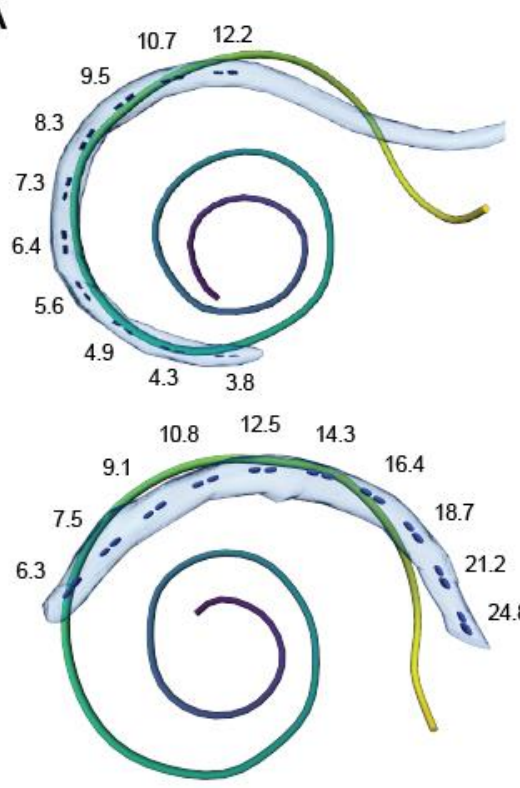

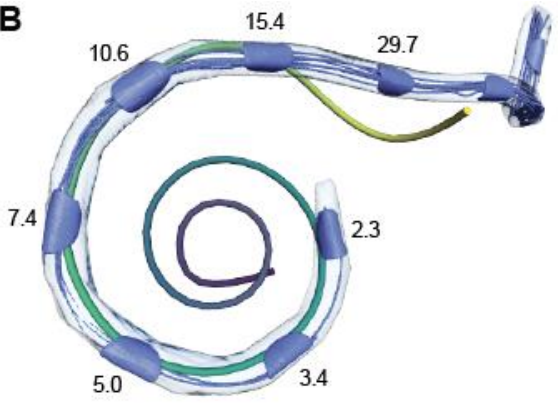

B

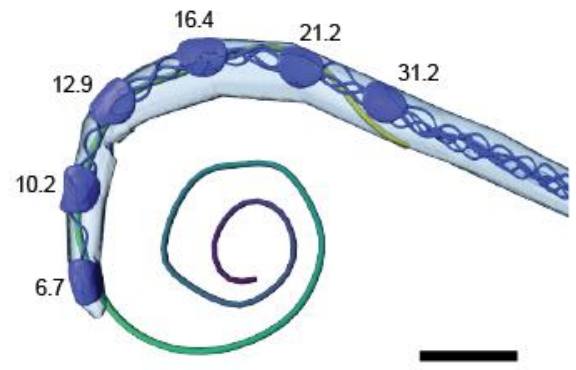

0.1

Frequency $(\mathrm{kHz})$

\section{Figure 4. Implantation trials in marmoset cochleae}

(A) Optical cochlear implant in marmoset scala tympani. LED bond pads (blue), spacing between LEDs $300 \mu \mathrm{m}$, silicone (bright blue), basilar membrane (color coded for corresponding frequency). View from cochlear base to apex. Top: Best example, insertion depth: $7.6 \mathrm{~mm}$. Bottom: insertion depth: $6.4 \mathrm{~mm}$. (B) Electrical cochlear implant in marmoset scala tymplani. Top: Best example, insertion depth: $8.9 \mathrm{~mm}$, spacing $1.5 \mathrm{~mm}$. Bottom: Insertion depth: $6 \mathrm{~mm}$, spacing $1 \mathrm{~mm}$. 
Table 2. Comparision of cochlear implant insertion depth across species

\begin{tabular}{lcccc}
\hline \multicolumn{1}{c}{ Species } & Mouse & Rat & Marmoset & Human \\
\hline \hline Scala tympani length (mm) & 6.3 & 7.1 & 14.8 & 26.0 \\
Scala tympani length (turns) & $2.00^{\mathrm{a}}$ & $2.25^{\mathrm{a}}$ & $2.75^{\mathrm{a}}$ & $2.60^{\mathrm{a}}$ \\
oCl insertion depth (mm) & 4.5 & 3.6 & 7.6 & - \\
oCl insertion depth (turns) & 1.43 & 1.14 & 1.41 & - \\
eCl insertion depth (mm) & $1.0-2.0^{\mathrm{b}}$ & $1.5-3.2^{\mathrm{c}}$ & 8.9 & $21.0^{\mathrm{d}}$ \\
eCl insertion depth (turns) & $0.32-0.63$ & $0.48-1.01$ & 1.65 & 2.10 \\
\hline \hline
\end{tabular}

a (West, 1985)

${ }^{b}$ (Irving et al., 2013; Mistry et al., 2014)

${ }^{c}$ (Argence et al., 2008; King et al., 2016; Lu et al., 2005)

${ }^{d}$ (Lee et al., 2010) 


\subsection{Discussion}

In this study, we quantitatively characterized commonly used temporal bones in auditory research on the basis of $\mu \mathrm{CT}$-derived models to enable design of an oCl array for use in rodents and marmosets. Xray tomography offered the possibility to precisely determine electrode and LED position within the scala tympani. The distinct advantage of 3D models is the possibility to have a digitized version readily available to be viewed from any angle. Further, virtual cross-sectioning gives valuable insight in cochlear structures and measurements that can easily be taken without manipulation of the original sample. However, soft tissue cannot easily be resolved and demonstrates a limitation of the method, thus, we employed lightsheet microscopy to be able to visualize histological details in 3D space.

Knowledge of cochlear fine structure enabled us to implant an optical probe for $4.5 \mathrm{~mm}$ in mice, doubling insertion depth previously reported (Table 2). Likewise, in rats the oCl array could be placed in lower frequency regions of $6.7 \mathrm{kHz}$ compared to maximum values known from literature. In case of a redesign of rat oCl similar to the one used in mice, using a dip coating rather than mold-injection encapsulation $(260 \mu \mathrm{m}$ vs. $340 \mu \mathrm{m}$ in mouse and rat oCl, respectively), insertion depth could potentially be further increased.

Implantation of eCls in marmosets exceeded previously reported insertion depth of $8 \mathrm{~mm}$ (Johnson et al., 2012) by $\sim 1 \mathrm{~mm}$ achieving a frequency-place range from $2.3-29.7 \mathrm{kHz}$ estimated with the Greenwood function according to an audible frequency range from (Osmanski and Wang, 2011). An improvement in upper limit coverage was possible through round window implantation, thus, making use of the high-frequency hook region of the cochlea.

Further, we now accomplished to implant 3 of 4 marmosets with specially designed 10 contact electrodes with an insertion depth of $12 \mathrm{~mm}$ estimating a lower frequency level of $920 \mathrm{~Hz}$. In one animal, we could position 7 contacts with an insertion depth of approximately $8 \mathrm{~mm}$.

Learning from scala tympani estimation, a more complex probe design could benefit the implantation depth further. Considering the curve progression of the height of scala tympani is mostly linear in all analyzed species with a negative slope of $76.5\left(R^{2}=0.78\right), 65.5\left(R^{2}=0.90\right), 57.0\left(R^{2}=0.87\right), 44.4\left(R^{2}=0.93\right)$, $33.1\left(R^{2}=0.91\right)$ for mouse, rat, gerbil, guinea pig, marmoset and human respectively, a tampered probe design could customize the shape to the cochlear specification. This could minimize damage to the sensitive scala tympani (Fan-Gang Zeng et al., 2008; Rebscher et al., 2008).

Here, we demonstrated deepest cochlear implantation depths reported for respective animal models (Table 2) with specially designed optical probes. Taking together ongoing development of multichannel oCls harboring $\mu$ LEDs (Klein et al., 2018), optogenetic stimulation of the auditory pathway (Duarte et al., 2018; Keppeler et al., 2018; Mager et al., 2018; Wrobel et al., 2018) and the findings of this study, the future for optical cochlear implants looks promising. 


\subsection{Methods}

\subsubsection{Animals}

Cochleae were obtained from sacrificed adult CD1 and C57BL/6N mice (Mus musculus), Wistar rats (Rattus norvegicus), gerbils (Meriones unguiculatus), Hartley guinea pigs (Cavia porcellus) and common marmosets (Callithrix jacchus). A human sample (doi:10.22016/smir.0.29498) was obtained from the SICAS Medical Image Repository (Kistler et al., 2013) under the CC BY 3.0 license. All experiments conformed to the local and national guidelines for the care and use of laboratory animals in research and were approved by the local authorities of the State of Lower Saxony (LAVES).

\subsubsection{Sample preparation}

Surgery for optical and electrical cochlear implant was performed on fresh marmoset skulls or sacrificed rodents. A small retro-auricular incision was made and overlaying muscles were translocated to expose the middle ear or in case of rodents the bulla. The probe was implanted via the round window and kept in place with instant adhesive. After preparation the samples were fixated in $4 \%$ paraformaldehyde until imaging. Known tissue shrinkage of paraformaldehyde between $2.9 \%$ and 4.5 \% (Fox et al., 1985; Jonmarker et al., 2006; O’Malley et al., 2009) was not corrected for.

\subsubsection{Cochlear implants}

Optical cochlear implants were designed with CreeTR2227 LEDs flip-chip bonded on flexible polyimide and subsequently dip-coated in silicone as described in (Schwaerzle et al., 2016) for marmosets. The optical probes contained 10 LEDs (size $220 \times 270 \mu \mathrm{m}$ ) spaced with $500 \mu \mathrm{m}$ and dip-coated in silicone (total diameter of approximately $260 \mu \mathrm{m}$ ). For rodents, we used integrated thin-film $\mu$ LEDs (size $50 \mu \mathrm{m}$ ) as described in (Ayub et al., 2016) with $93 \mu \mathrm{LEDs}$, spaced at $50 \mu \mathrm{m}$, tapered from $194 \mu \mathrm{m}$ to $160 \mu \mathrm{m}$, encapsulated in silicone (oval profile approx. 240×130 $\mu \mathrm{m}$ ) and $13 \mu \mathrm{LEDs}$, spaced at $300 \mu \mathrm{m}$, encapsulated in silicone (round profile $340 \mu \mathrm{m}$ ) for mouse and rat, respectively. Electrical cochlear implants were kindly provided by Med-El in two different designs: Electrode spacing was $1000 \mu \mathrm{m}$ and $1500 \mu \mathrm{m}$, wires wriggled or straight for probe 1 and 2, respectively. Electrode diameter was $500 \mu \mathrm{m}$.

\subsubsection{MicroCT}

Anatomical and morphological features in animal models were assessed with phase-contrast tomography at a compact laboratory X-ray source essentially as previously described (Bartels et al., 2013). The custom-built cone-beam in-line tomography instrument equipped with a liquid-metal X-ray source (JXS D2, Excillum) operated at $70 \mathrm{kV}$ acceleration voltage was combined with a $20 \mu \mathrm{m}$ LuAGscintillator-based fiber-coupled CCD detector (6.5 $\mu \mathrm{m}$ pixel size; C12849-102U; Hamamatsu). Fast 
Fourier-based phase reconstruction was used to calculate the image volume. Reconstruction of the raw data was performed using the ASTRA toolbox (van Aarle et al., 2015, 2016). Voxel dimensions were isotropic, ranging from 2.00-6.63 $\mu \mathrm{m}$.

\subsubsection{Image analysis}

Segmentation, 3D visualization of the reconstructed volume, measurements and analysis were performed with Avizo (FEI Visualization Sciences Group, Thermo Fisher Scientific Inc.). The raw dataset was initially visualized as volume rendering. Each point in the data volume is represented by scalar data using a user-defined colormap including alpha values. For segmentation, the $\mu \mathrm{CT}$ cross-sections were viewed in three orthogonal planes. Automatic histogram-based segmentation was used to visualize bony structures. The basilar membrane, Rosenthal's canal, osseous spiral lamina, modiolus, scala tympani and scala vestibuli et media were traced with semiautomatic segmentation. In most cases soft tissue structures like the Reissner's membrane could not be resolved. Thereby, the scala media could not be separated from scala vestibule and, thus, both cavities were lumped together in one fluid space. The volume of surfaces was determined based on a voxel count of the reconstructed spaces. The centerline was calculated with Avizo tools implementing the TEASAR algorithm originally described in (Sato et al., 2000). Also, the Euclidean distance to the nearest boundary was determined for every point of the spatial graph (591-1762 sampling points depending on species) giving an estimation for the radius. Subsequently the centerline tree was reduced to the relevant branch and smoothed with a moving average filter. In case of the scala tympani the centerline was used as a trajectory for virtual cross-sections (20-47 sampling points depending on species) along the spiral cavity. The cross-sectional area, width and height of the scalae were measured using standard Avizo tools. The width was determined in parallel to the osseous spiral lamina at the maximum distance between the lateral and medial wall. The height was also measured at its maximum, perpendicular to the width. The length of the basilar membrane was determined with a fitted spline along the centerline between lateral and medial edge. The insertion depth of $\mathrm{Cls}$ was measured with a fitted spline curve to markers along the device.

\subsubsection{Data analysis}

Parameter of the scala tympani (radius, height, width, curvature) were scaled along the distance from base dimension to fit the mean length of the scala tympani per species, interpolated to retrieve 100 linearly spaced query points and subsequently averaged. For the curvature, the Euclidean distance between centerline and modiolar axis was determined for every sample point along the spline. Frequency-place estimates were derived from the Greenwood function (Greenwood, 1961, 1990) and fitted along the basilar membrane. Estimated audible frequency ranges were taken from (West, 1985). 
Multiscale, multimodal imaging of the native and implanted cochlea

For estimation of the corresponding frequency of individual stimulation nearest neighbor search to the basilar membrane was used. Data were reported with standard error of the mean if not stated differently. 


\subsection{Supplements}
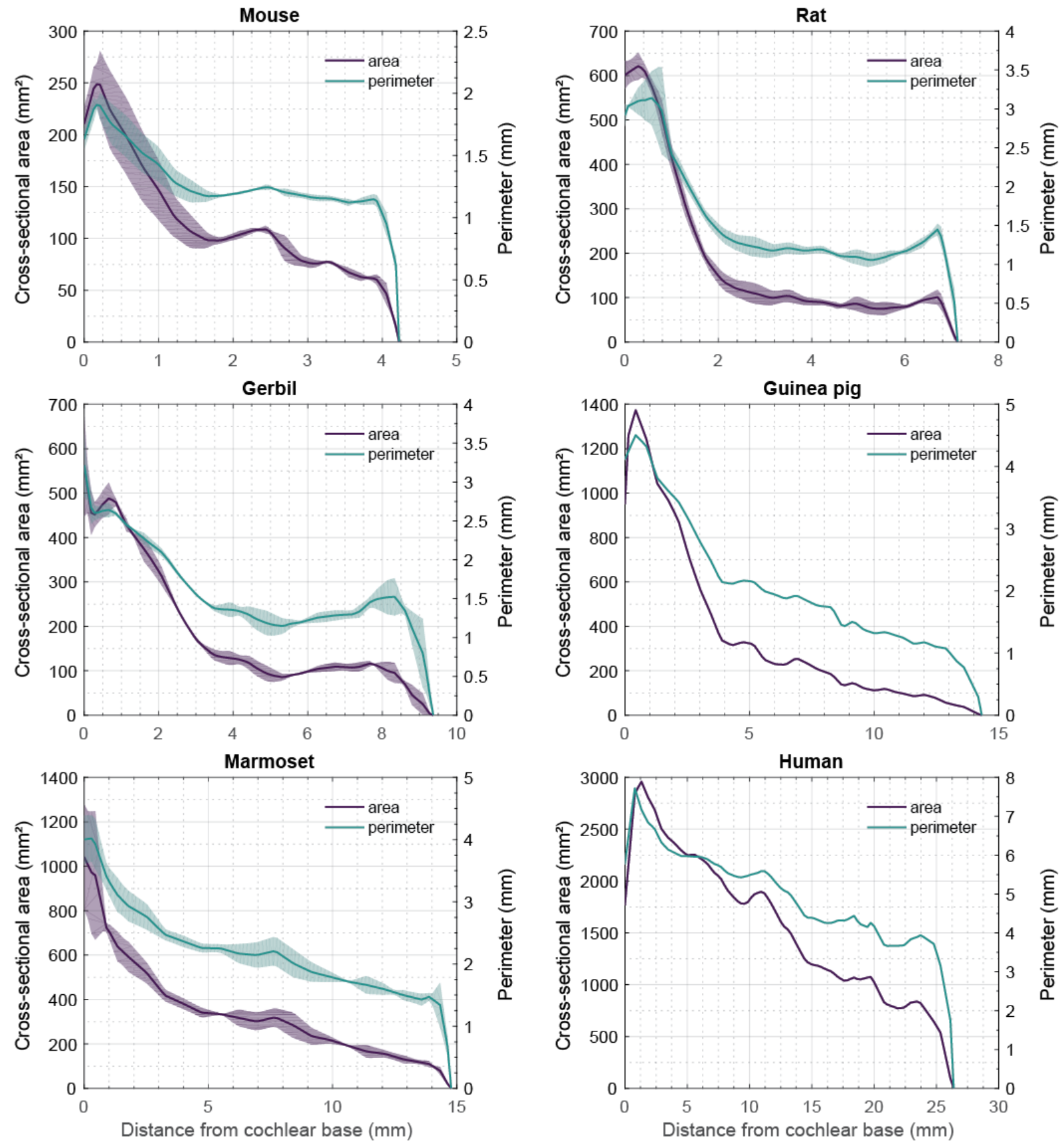

Figure S1. Cross-sectional area and perimeter

Measurements were obtained from virtual cross-sections along the cochlea. Please note the two different ordinates for area and perimeter. 


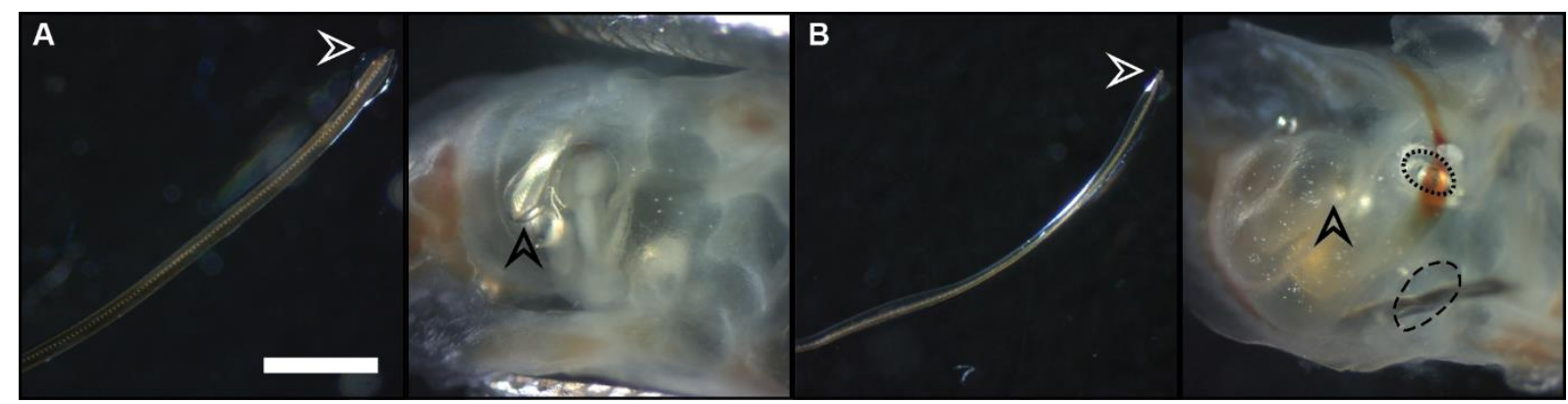

Figure S2. Implantation trials of dip-coated polyimide-based probes in mouse cochleae

Polyimide-based probes were encapsulated via dip-coating into silicone and thereafter spun in a custom-built centrifuge. Two ex vivo implantation examples in translucent mouse cochleae through prior embedding in 2,2-thiodiethanl (TDE):

(A) Suboptimal silicone layer with droplet formation at the tip (arrow, left and right panel). Right panel depicts implantation of encapsulated probe with an insertion depth of $1.8 \mathrm{~mm}$. The tip of the probe was stuck after approximately half a turn at the lateral wall of scala tympani (view from medial side).

(B) Optimal silicone layer without droplet formation (left panel). An implantation depth of $3.8 \mathrm{~mm}$ was achieved via the round window (dashed line). The tip of the probe (arrow) reached one turn in the scala tympani. Oval window (dotted line). Scale bar $1 \mathrm{~mm}$. 


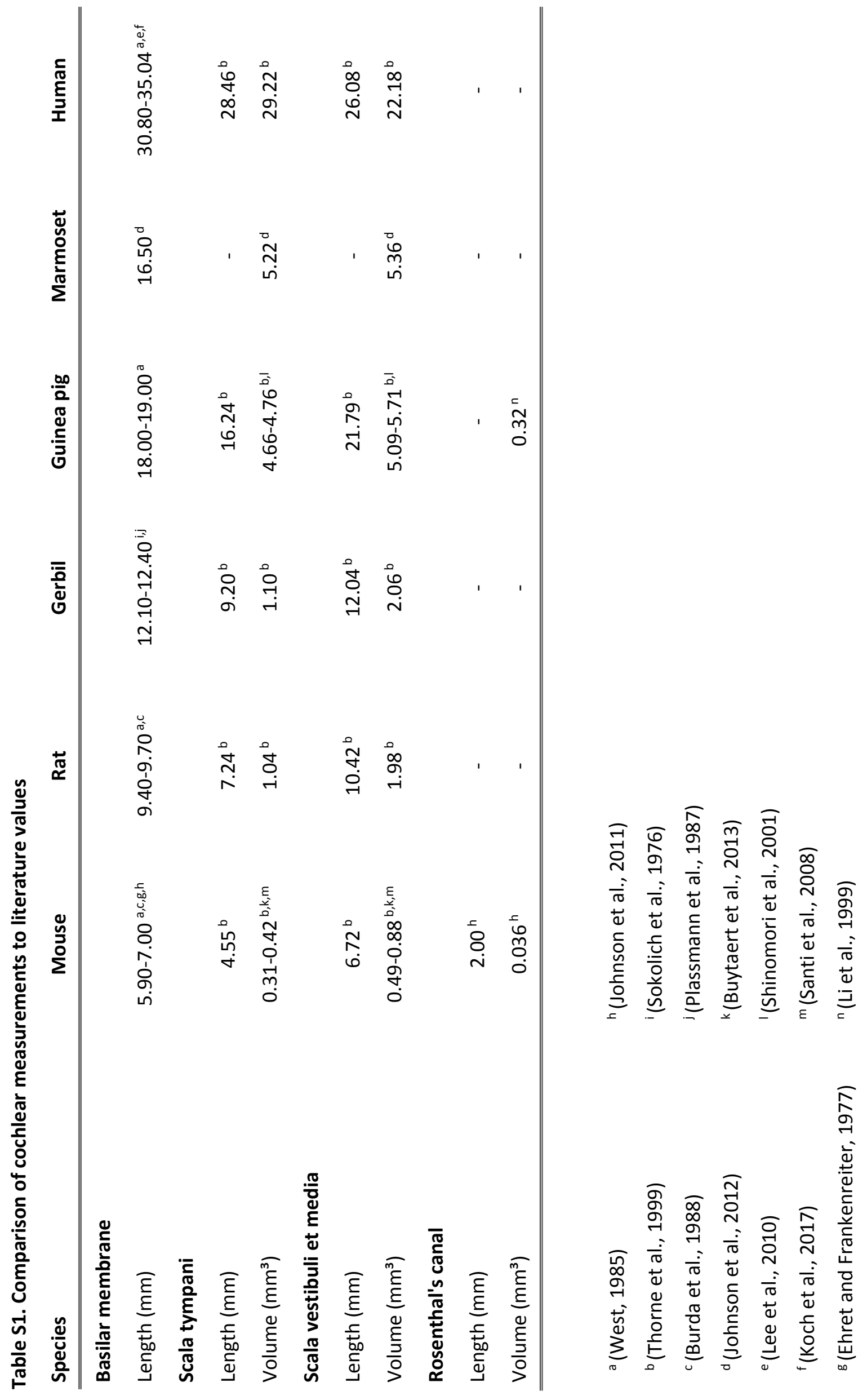




\subsection{References}

van Aarle, W., Palenstijn, W.J., De Beenhouwer, J., Altantzis, T., Bals, S., Batenburg, K.J., and Sijbers, J. (2015). The ASTRA Toolbox: A platform for advanced algorithm development in electron tomography. Ultramicroscopy 157, 35-47.

van Aarle, W., Palenstijn, W.J., Cant, J., Janssens, E., Bleichrodt, F., Dabravolski, A., Beenhouwer, J.D., Batenburg, K.J., and Sijbers, J. (2016). Fast and flexible X-ray tomography using the ASTRA toolbox. Opt. Express 24, 25129-25147.

Argence, M., Vassias, I., Kerhuel, L., Vidal, P.-P., and Waele, C.D. (2008). Stimulation by cochlear implant in unilaterally deaf rats reverses the decrease of inhibitory transmission in the inferior colliculus. Eur. J. Neurosci. 28, 1589-1602.

Ayub, S., Gossler, C., Schwaerzle, M., Klein, E., Paul, O., Schwarz, U.T., and Ruther, P. (2016). Highdensity probe with integrated thin-film micro light emitting diodes (microLEDs) for optogenetic applications. In 2016 IEEE 29th International Conference on Micro Electro Mechanical Systems (MEMS), (IEEE), pp. 379-382.

Bartels, M., Hernandez, V.H., Krenkel, M., Moser, T., and Salditt, T. (2013). Phase contrast tomography of the mouse cochlea at microfocus x-ray sources. Appl. Phys. Lett. 103, 083703.

Burda, H., Ballast, L., and Bruns, V. (1988). Cochlea in old world mice and rats (Muridae). J. Morphol. 198, 269-285.

Buytaert, J.A., Johnson, S.B., Dierick, M., Salih, W.H., and Santi, P.A. (2013). MicroCT versus sTSLIM 3D imaging of the mouse cochlea. J. Histochem. Cytochem. 0022155413478613.

Dombrowski, T., Rankovic, V., and Moser, T. (2018). Toward the Optical Cochlear Implant. Cold Spring Harb Perspect Med. doi: 10.1101/cshperspect.a033225

Duarte, M.J., Kanumuri, V.V., Landegger, L.D., Tarabichi, O., Sinha, S., Meng, X., Hight, A.E., Kozin, E.D., Stankovic, K.M., Brown, M.C., et al. (2018). Ancestral Adeno-Associated Virus Vector Delivery of Opsins to Spiral Ganglion Neurons: Implications for Optogenetic Cochlear Implants. Molecular Therapy 26, 1931-1939.

Ehret, G., and Frankenreiter, M. (1977). Quantitative analysis of cochlear structures in the house mouse in relation to mechanisms of acoustical information processing. J. Comp. Physiol. 122, 65-85.

Fox, C.H., Johnson, F.B., Whiting, J., and Roller, P.P. (1985). Formaldehyde fixation. J. Histochem. Cytochem. 33, 845-853.

Goßler, C., Bierbrauer, C., Moser, R., Kunzer, M., Holc, K., Pletschen, W., Köhler, K., Wagner, J., Schwaerzle, M., Ruther, P., et al. (2014). GaN-based micro-LED arrays on flexible substrates for optical cochlear implants. J. Phys. Appl. Phys. 47, 205401.

Greenwood, D.D. (1961). Auditory Masking and the Critical Band. J. Acoust. Soc. Am. 33, 484-502.

Greenwood, D.D. (1990). A cochlear frequency-position function for several species--29 years later. J. Acoust. Soc. Am. 87, 2592-2605.

Hernandez, V.H., Gehrt, A., Reuter, K., Jing, Z., Jeschke, M., Mendoza Schulz, A., Hoch, G., Bartels, M., Vogt, G., Garnham, C.W., et al. (2014). Optogenetic stimulation of the auditory pathway. J. Clin. Invest. $124,1114-1129$. 
Irving, S., Trotter, M.I., Fallon, J.B., Millard, R.E., Shepherd, R.K., and Wise, A.K. (2013). Cochlear implantation for chronic electrical stimulation in the mouse. Hear. Res. 306, 37-45.

Jeschke, M., and Moser, T. (2015). Considering optogenetic stimulation for cochlear implants. Hear. Res. 322, 224-234.

Johnson, L.A., Della Santina, C.C., and Wang, X. (2012). Temporal bone characterization and cochlear implant feasibility in the common marmoset (Callithrix jacchus). Hear. Res. 290, 37-44.

Johnson, S.B., Schmitz, H.M., and Santi, P.A. (2011). TSLIM imaging and a morphometric analysis of the mouse spiral ganglion. Hear. Res. 278, 34-42.

Jonmarker, S., Valdman, A., Lindberg, A., Hellström, M., and Egevad, L. (2006). Tissue shrinkage after fixation with formalin injection of prostatectomy specimens. Virchows Arch. Int. J. Pathol. 449, 297301.

Kang, R., Nimmons, G.L., Drennan, W., Longnion, J., Ruffin, C., Nie, K., Won, J.H., Worman, T., Yueh, B., and Rubinstein, J. (2009). Development and Validation of the University of Washington Clinical Assessment of Music Perception Test. Ear Hear. 30, 411-418.

King, J., Shehu, I., Roland, J.T., Svirsky, M.A., and Froemke, R.C. (2016). A physiological and behavioral system for hearing restoration with cochlear implants. J. Neurophysiol. 116, 844-858.

Kistler, M., Bonaretti, S., Pfahrer, M., Niklaus, R., and Büchler, P. (2013). The Virtual Skeleton Database: An Open Access Repository for Biomedical Research and Collaboration. J. Med. Internet Res. 15, e245.

Klein, E., Gossler, C., Paul, O., and Ruther, P. (2018). High-density $\mu$ LED-based Optical Cochlear Implant with Improved Thermomechanical Behavior. Front. Neurosci. 12.

Koch, R.W., Ladak, H.M., Elfarnawany, M., and Agrawal, S.K. (2017). Measuring Cochlear Duct Length - a historical analysis of methods and results. J. Otolaryngol. - Head Neck Surg. 46, 19.

Kral, A., Hartmann, R., Mortazavi, D., and Klinke, R. (1998). Spatial resolution of cochlear implants: the electrical field and excitation of auditory afferents. Hear. Res. 121, 11-28.

Laubsch, A., Sabathil, M., Baur, J., Peter, M., and Hahn, B. (2010). High-Power and High-Efficiency InGaN-Based Light Emitters. IEEE Trans. Electron Devices 57, 79-87.

Lee, J., Nadol, Jr., J.B., and Eddington, D.K. (2010). Depth of Electrode Insertion and Postoperative Performance in Humans with Cochlear Implants: A Histopathologic Study. Audiol. Neurotol. 15, 323331.

Li, L., Parkins, C.W., and Webster, D.B. (1999). Does electrical stimulation of deaf cochleae prevent spiral ganglion degeneration? Hear. Res. 133, 27-39.

Li, L., Parkins, C.W., and Webster, D.B. (1999). Does electrical stimulation of deaf cochleae prevent spiral ganglion degeneration? Hear. Res. 133, 27-39.

Mager, T., Morena, D.L. de la, Senn, V., Schlotte, J., D’Errico, A., Feldbauer, K., Wrobel, C., Jung, S., Bodensiek, K., Rankovic, V., et al. (2018). High frequency neural spiking and auditory signaling by ultrafast red-shifted optogenetics. Nat. Commun. 9, 1750.

Mistry, N., Nolan, L.S., Saeed, S.R., Forge, A., and Taylor, R.R. (2014). Cochlear implantation in the mouse via the round window: Effects of array insertion. Hear. Res. 312, 81-90. 
O'Malley, J.T., Merchant, S.N., Burgess, B.J., Jones, D.D., and Adams, J.C. (2009). Effects of Fixative and Embedding Medium on Morphology and Immunostaining of the Cochlea. Audiol. Neurootol. 14, 7887.

Osmanski, M.S., and Wang, X. (2011). Measurement of absolute auditory thresholds in the common marmoset (Callithrix jacchus). Hear. Res. 277, 127-133.

Plassmann, W., Peetz, W., and Schmidt, M. (1987). The Cochlea in Gerbilline Rodents. Brain. Behav. Evol. 30, 82-102.

Santi, P.A., Rapson, I., and Voie, A. (2008). Development of the mouse cochlea database (MCD). Hear. Res. 243, 11-17.

Sato, M., Bitter, I., Bender, M.A., Kaufman, A.E., and Nakajima, M. (2000). TEASAR: tree-structure extraction algorithm for accurate and robust skeletons. In Proceedings the Eighth Pacific Conference on Computer Graphics and Applications, pp. 281-449.

Schwaerzle, M., Nehlich, J., Ayub, S., Paul, O., Patrick, R., and others (2016). Led-based optical cochlear implant on highly flexible triple layer polyimide substrates. In 2016 IEEE 29th International Conference on Micro Electro Mechanical Systems (MEMS), (IEEE), pp. 395-398.

Shinomori, Y., Jones, D. d., Spack, D.S., and Kimura, R.S. (2001). Volumetric and Dimensional Analysis of the Guinea Pig Inner Ear. Ann. Otol. Rhinol. Laryngol. 110, 91-98.

Sokolich, W.G., Hamernik, R.P., Zwislocki, J.J., and Schmiedt, R.A. (1976). Inferred response polarities of cochlear hair cells. J. Acoust. Soc. Am. 59, 963-974.

Song, X., Osmanski, M.S., Guo, Y., and Wang, X. (2016). Complex pitch perception mechanisms are shared by humans and a New World monkey. Proc. Natl. Acad. Sci. U. S. A. 113, 781-786.

Thorne, M., Salt, A.N., DeMott, J.E., Henson, M.M., Henson, O.W., and Gewalt, S.L. (1999). Cochlear fluid space dimensions for six species derived from reconstructions of three-dimensional magnetic resonance images. The Laryngoscope 109, 1661-1668.

Tomita, H., Sugano, E., Fukazawa, Y., Isago, H., Sugiyama, Y., Hiroi, T., Ishizuka, T., Mushiake, H., Kato, M., Hirabayashi, M., et al. (2009). Visual Properties of Transgenic Rats Harboring the Channelrhodopsin-2 Gene Regulated by the Thy-1.2 Promoter. PLOS ONE 4, e7679.

West, C.D. (1985). The relationship of the spiral turns of the cochlea and the length of the basilar membrane to the range of audible frequencies in ground dwelling mammals. J. Acoust. Soc. Am. 77, 1091-1101.

Witten, I.B., Steinberg, E.E., Lee, S.Y., Davidson, T.J., Zalocusky, K.A., Brodsky, M., Yizhar, O., Cho, S.L., Gong, S., Ramakrishnan, C., et al. (2011). Recombinase-driver rat lines: tools, techniques, and optogenetic application to dopamine-mediated reinforcement. Neuron 72, 721-733.

Wrobel, C., Dieter, A., Huet, A., Keppeler, D., Duque-Afonso, C.J., Vogl, C., Hoch, G., Jeschke, M., and Moser, T. (2018). Optogenetic stimulation of cochlear neurons activates the auditory pathway and restores auditory-driven behavior in deaf adult gerbils. Sci. Transl. Med. 10, eaao0540.

Zeng, H., and Madisen, L. (2012). Mouse transgenic approaches in optogenetics. Prog. Brain Res. 196, 193-213. 


\subsection{Acknowledgments}

We thank Patrick Ruther and Ulrich T. Schwarz for providing optical cochlear implants. We thank Roland Hessler from MED-EL Company (Innsbruck, Austria) for providing electrical cochlear implants. We thank Marcus Jeschke for help with the custom-written Greenwood function script.

Funding: This work was funded by the European Research Council through the Advanced Grant 'OptoHear" to T.M. under the European Union's Horizon 2020 Research and Innovation program (grant agreement No. 670759) and was further supported by the German Ministry of Research and Education through the Bernstein Center for Computational Neuroscience to T.M. (under grant number 01GQ1005A) and the grant Optical Cl (under grant number 13N13729) to T.M.) as well as the German Research Foundation (through the DFG-Research Center (FZT103) and Cluster of Excellence (EXC170) Center for Nanoscale Microscopy and Molecular Physiology of the Brain to T.M., as well as the Leibniz Program to T.M.

Competing Interests: The authors declare no competing interests. 


\section{Towards optical cochlear implants: First multichannel optogenetic stimulation of the auditory pathway using microfabricated LED implants}

\section{Authors:}

Daniel Keppeler ${ }^{1,2^{*}}$, Michael Schwaerzle ${ }^{3,4^{*}}$, Julian Nehlich ${ }^{3}$, Tamas Harczos ${ }^{1,5}$, Christian Wrobel $^{1,6, \$}$, Patrick Ringwald ${ }^{3,4}$, Roiy Raz ${ }^{3}$, Gerhard Hoch ${ }^{1,5}$, Khaled Abdellatif ${ }^{1,4,6}$, Kai Bodensiek ${ }^{1}$, Oliver Paul ${ }^{3,4}$, Patrick Ruther ${ }^{3,4, \#,}$, Tobias Moser ${ }^{1,2,5,6,7, \#}$

\section{Affiliations:}

${ }^{1}$ Auditory Neuroscience and InnerEarLab, University Medical Center Göttingen, Germany.

${ }^{2}$ Göttingen Graduate School for Neurosciences and Molecular Biosciences, University of Göttingen, Germany.

${ }^{3}$ University of Freiburg, Department of Microsystems Engineering (IMTEK), Freiburg, Germany

${ }^{4}$ Cluster of Excellence Brainlinks-BrainTools, University of Freiburg, Germany

${ }^{5}$ Auditory Neuroscience and Optogenetics Group, German Primate Center, Göttingen, Germany

${ }^{6}$ Collaborative Research Center 889, University of Göttingen, Germany.

${ }^{7}$ Center for Nanoscale Microscopy and Molecular Physiology of the Brain, University Medical Center Göttingen, Germany.

${ }^{\S}$ present address: Department of Otorhinolaryngology, Head and Neck Surgery, Ruhr University Bochum, St. Elisabeth Hospital, Bochum, Germany.

*equal contribution

"To whom correspondence should be addressed:

Dr. Patrick Ruther, Email: ruther@imtek.de

Dr. Tobias Moser, Email: tmoser@gwdg.de 


\section{Author contributions:}

This study was designed by T.M., P.R., D.K. and M.S., M.S. and D.K. developed the oCls and performed the experiments, analyzed the measurement data, prepared the figures, and contributed the manuscript with equal effort; C.W. contributed to OABR recordings and analysis. P.R. realized the photolithography masks of the PI substrates; T.H., K.A. and G.H. established the stimulation set-up for the in vivo experiments, D.K., K.B., K.A., T.H. and G.H. characterized the probes before and after physiology experiments; J.N. supported M.S. during thermal probe characterization and development of the thermal model; R.R. performed the passivation long-term tests; T.M., P.R., M.S. and D.K. prepared the manuscript; M.S., T.M., O.P. and P.R. discussed the results and finalized the manuscript. All authors reviewed the manuscript.

Detailed author contributions of D. Keppeler (D.K.):

- Experimental work

○ X-ray tomography

- Implantation of oCls

- Electrophysiological recordings

- Analysis
- 3D quantification of segmented data
- Evaluation of implant position
- Electrophysiological data
- Statistics

- Preparation of the manuscript (together with T.M., M.S. and P.R.) 


\subsection{One Sentence Summary}

We report the design, fabrication, and technical characterization of highly flexible LED-arrays and their successful application for optogenetic stimulation of the auditory pathway.

\subsection{Abstract}

When hearing fails, cochlear implants $(\mathrm{Cls})$ provide the brain with auditory information that enables speech understanding in the majority of the approximately 0.5 million users. One important bottleneck of $\mathrm{Cls}$ is the poor spectral resolution of coding that results from the wide current spread throughout the cochlea from each of the electrode contacts. Optical Cls (oCls) promise to make better use of the tonotopic order of spiral ganglion neurons (SGNs) inside the cochlea by more focal stimulation. Here we established multichannel oCls based on microfabricated light-emitting diode (LED) arrays and employed them for optical stimulation of channelrhodopsin-2 (ChR2)-expressing SGNs in transgenic rats. Power-efficient blue LED chips $\left(220 \times 270 \mu \mathrm{m}^{2}, 454 \mathrm{~nm}\right.$ emission) were integrated at a pitch of 350 or $500 \mu \mathrm{m}$ onto $15-\mu \mathrm{m}$-thin polyimide-based carriers comprising interconnecting lines that addressed individual LEDs. Thin-film passivation using a fluoropolymer was followed by silicone encapsulation. We extensively characterized the optoelectronic, thermal and mechanical properties of the oCls and demonstrated stability over weeks. Finally, we implanted oCls into ChR2-expressing transgenic rats and studied optical auditory brainstem responses (OABR) stimulated by individual or multiple LEDs. The oCls could be advanced into scala tympani to cover the basal cochlear turn, as demonstrated by $X$-ray tomography. In conclusion, the study demonstrates feasibility of LED-based multi-channel oCls.

\subsection{Introduction}

Hearing impairment $(\mathrm{HI})$ is the most common sensory deficit and approximately $5 \%$ of the world's population suffers from disabling $\mathrm{HI}(1)$. Despite the dramatic clinical need and major research activities, a causal therapy is not yet available for the most common form: sensorineural HI (2-4). Hence, hearing aids and $\mathrm{Cls}$, once $\mathrm{HI}$ has reached severe state, will remain the key means for partial restoration of hearing. Cls use only 12-24 electrode contacts along the tonotopic map of the human cochlea, which in normal hearing allows discrimination of approximately 2000 frequency steps (5), and cover a frequency range of approximately $100 \mathrm{~Hz}$ to $10 \mathrm{kHz}$ (6). Moreover, the spread of current from each electrode contact (7) leads to channel crosstalk (8) and diminishes the number of independent stimulation channels $(9,10)$. Optogenetic stimulation of the cochlea is a promising approach to increase the spectral resolution of $\mathrm{Cl}$ coding, as the cochlear spread of excitation was shown to be lower for light emitted from an optical fiber with a core diameter of $250 \mu \mathrm{m}$ than with monopolar electrical stimulation (11). However, much remains to be done in order to develop optical stimulation 
as an alternative operating mode in clinical Cls (12). Next to establishing virus-mediated expression of opsins in auditory neurons $(11,13,14)$, multichannel oCls need to be developed. Generally, active and passive optical probes might be implanted into the cochlea (12). While waveguide arrays would passively propagate light from sources outside the cochlea, e.g. from within the sealed housing of the $\mathrm{Cl}$ stimulator, active optoelectronic probes would convert current to light in encapsulated microscale emitters inside the cochlea. Interesting candidate emitters for blue optogenetic applications at the systems level are gallium-nitride (GaN) based micro LEDs ( $\mu \operatorname{LEDs},(12,15,16))$. These $\mu$ LEDs can be integrated on flexible substrates using various approaches (17-19). So far, light sources used for optogenetic stimulation of SGNs have been GaN LEDs and optical fibers coupled to an external laser. Both LEDs or fibers were either placed onto a cochleostomy or into the cochlear scala tympani via the round window (11) and were limited to a single stimulation channel. A fiber-based single channel approach was also demonstrated for photoactivation of auditory brainstem and midbrain neurons expressing either channelrhodopsin-2 (ChR2) or Chronos $(13,14)$. Clearly, single-channel optical stimulation has been an important step towards establishing the optogenetic control of the auditory pathway. However, developing oCls for auditory research and future clinical translation requires the generation of multichannel devices. We have recently reported the wafer-level integration of GaNbased $\mu$ LEDs into flexible high-density arrays carrying $100 \mu$ LEDs with a size of $50 \times 50 \mu \mathrm{m}^{2}$ on a substrate with a length of $10 \mathrm{~mm}$ that fits into the cochlea of mice (18). However, the custom-made $\mu$ LEDs were not yet optimal in terms of radiant flux (sub-mW) and wavelength (405 nm) required for the activation of $\mathrm{ChR} 2$, and robust electrical connection as well as encapsulation of the probe remained to be established prior to their functional characterization.

Here, we established and characterized LED-arrays as multichannel oCls integrating larger and highly power-efficient commercial GaN-based LED chips integrated on a polyimide carrier. We study electrical, optical and thermal properties and optimized the electrical passivation and mechanical properties as required for an application in the cochlea. The oCl encapsulation employed thin-film passivation using the fluoropolymer $\mathrm{Cytop}^{\mathrm{TM}}$, which lent the oCl stability in saline solution for weeks of operation with negligible leakage currents between the interconnecting lines. When further encapsulated in silicone, these oCls were compatible with the use in the cochlea of larger rodents such as the rat. Using transgenic rats expressing ChR2-Venus under the pan-neuronal promoter Thy1.2 (20), we performed a first characterization of insertion and functional stimulation. This work paves the way for acute and chronic studies of optogenetic stimulation of the cochlea but will likely also aid optogenetic studies in other systems. A preliminary conference report on the technical development of these tools has been presented recently (21). 


\subsection{Results}

\subsubsection{Design of LED-based optical cochlear implants (oCls)}

In order to design oCls with appropriate dimensions and mechanical properties, we first performed Xray tomography of the rat cochlea (22) yielding estimates of the cross-section of scala tympani and its curvature (Fig. 1A and B). The cross-section starts out with a width of near $1000 \mu \mathrm{m}$ at the cochlear base, drops to approximately $300 \mu \mathrm{m}$ in the mid-cochlea and then levels off. Likewise, the radius of curvature starts out large (approximately $1.4 \mathrm{~mm}$ ) and declines to about $400 \mu \mathrm{m}$ in the apex. Hence, our target was a slim and flexible array not exceeding $300 \mu \mathrm{m}$ in diameter after encapsulation and being compatible, both mechanically as well as functionally, with a bending radius of approximately $500 \mu \mathrm{m}$.

The design of the oCls based on a polyimide (PI) substrate and commercially available LED chips emitting at $454 \mathrm{~nm}$ is illustrated in Fig. 1C. It consists of a slender implantable section (width $240 \mu \mathrm{m}$ ) with a linear array of LEDs at its tip wired to the interface section designed for zero insertion force (ZIF) connectors. The central 11 contact pads of the ZIF connector section and the respective wiring lines (a broader common $n$-contact line and ten p-contact lines, one for each LED, inset in Fig. 1C), address individual LEDs for their independent control. The outer three pads on each side of the ZIF interface were short-circuited, hence allowing a connection test of the electrical interface in the case of these first oCl prototypes. The linear array of ten LEDs at the oCl tip was designed with a pitch of $500 \mu \mathrm{m}$ or $350 \mu \mathrm{m}$ (see Fig. 1C). In addition, an array with four LEDs containing a temperature sensor based on a platinum meander integrated beneath the first LED chip was established (see Fig. 1D). The four-wire interconnection of this meander enables a precise resistance measurement and thus probe temperature determination while operating the LED chips. 

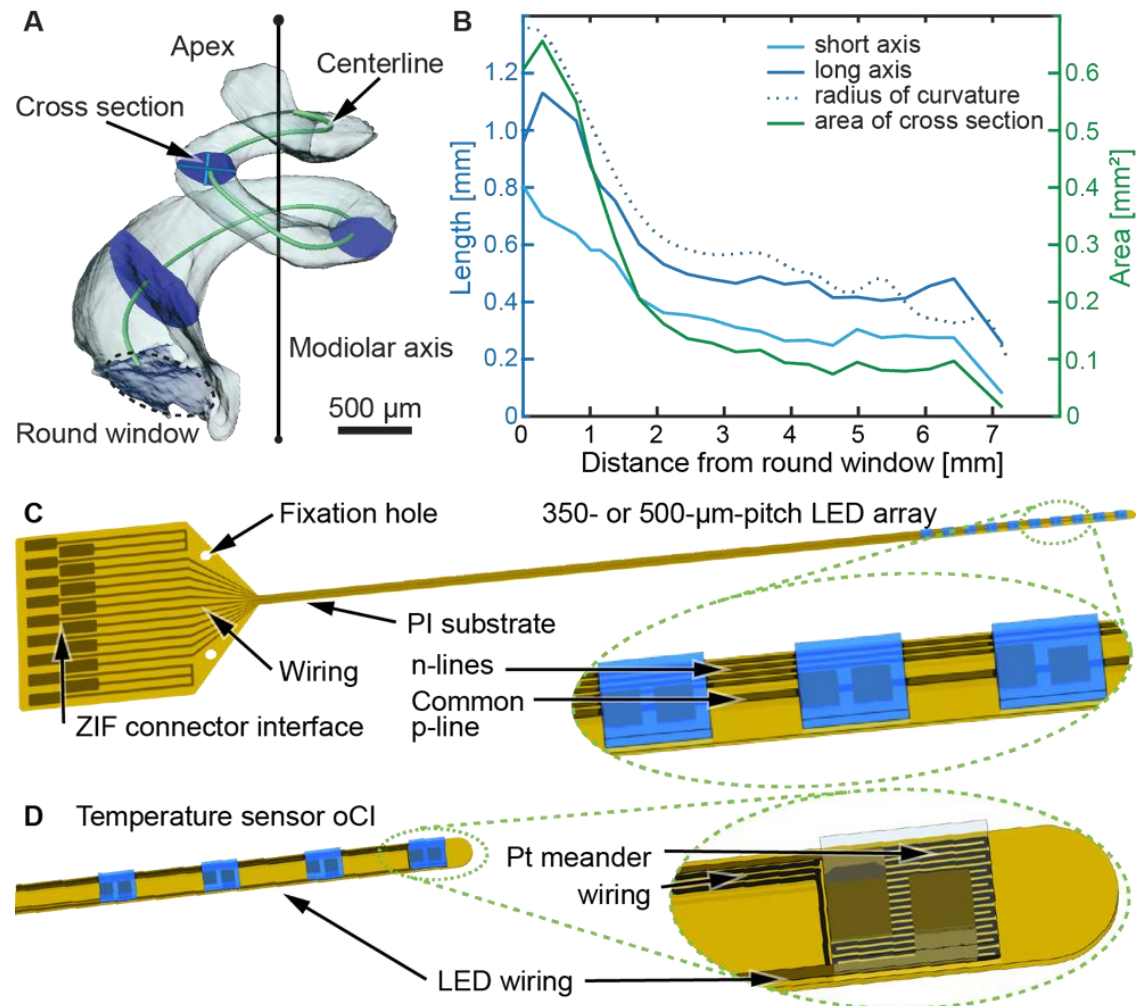

E $\quad 350-\mu \mathrm{m}$-pitch LED array
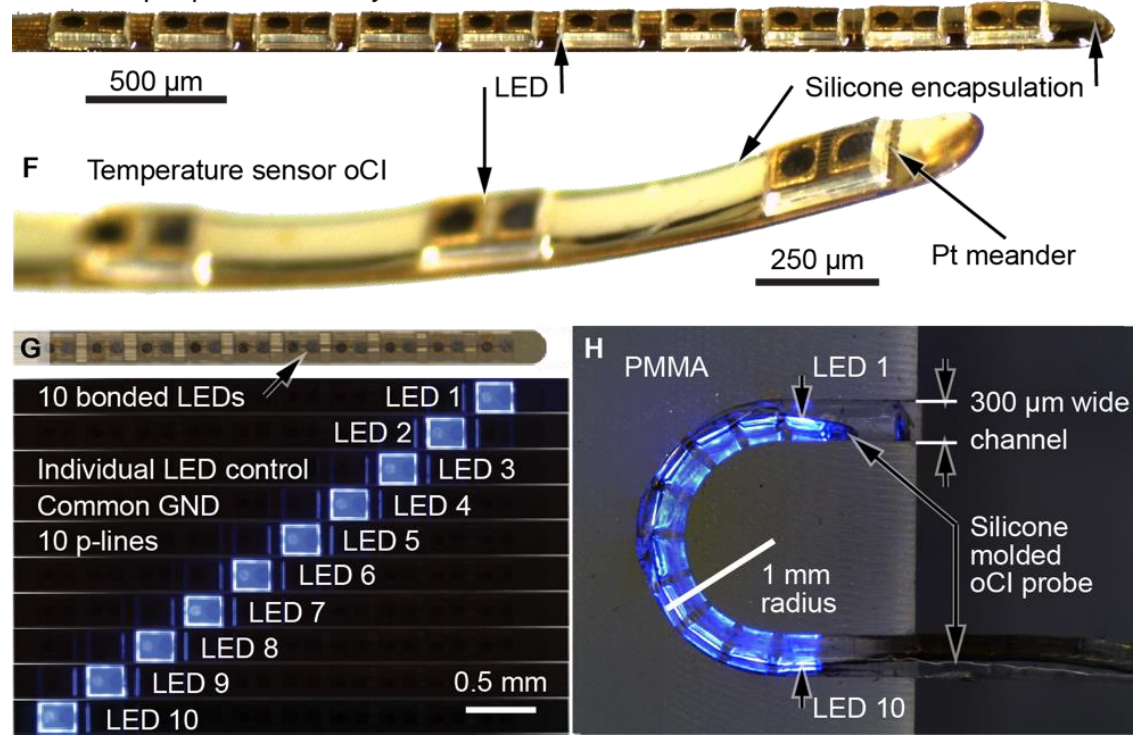

Figure 1. Design of the LED-based multichannel optical cochlear implants.

(A) Reconstruction of the scala tympani of a rat based on X-ray tomography, with the round window at the bottom and cochlear apex pointing up. Green line: center of scala tympani, blue areas: the exemplary cross-sections, blue lines: short and long axes of the cross-sections, black line: modiolar axis of the cochlea subjectively derived from the X-ray data.

(B) Radius of curvature (dotted line), short (light blue) and long (dark blue) axes of cross-sections (area in green, right axis) as a function of distance from the round window.

(C) Design of the highly flexible PI-based oCl with a linear array of ten LED chips integrated at the tip of the slender implantable section of the optical probe. The LEDs are individually wired and connected via a zero insertion force (ZIF) interface at the wider connector part comprising fixation holes for optional device attachment to the skull.

(D) Design of the probe tip with resistive temperature sensor integrated beneath the first of four LED chips that were arranged at a pitch of $750 \mu \mathrm{m}$. The platinum meander of the temperature sensing element is connected via four wires for a precise resistance measurement. 
(E, F) Optical micrographs of fabricated and silicone-encapsulated oCls with (E) ten LED chips assembled at an LED pitch of $350 \mu \mathrm{m}$ and (F) with an integrated temperature sensor beneath the first LED with an LED pitch of $750 \mu \mathrm{m}$.

(G) Functionality test of an oCl demonstrating the individual control of LEDs prior to device passivation.

(H) Bending test of a silicone-molded oCl inserted into a 300- $\mu \mathrm{m}$-wide channel with an outer radius of $1 \mathrm{~mm}$ milled into a PMMA substrate.

$(C, D, G)$ Copyright $@$ IEEE. All rights reserved. Reprinted, with permission, from [20].

\subsubsection{Fabrication, assembly and passivation}

The fabrication of the oCls started by generating the PI-based carrier on a silicon (Si) handle wafer. The microfabrication steps, as detailed in the Supplementary Material, included the subsequent spin coating and curing of three PI layers patterned by reactive ion etching (RIE) sequences, the sputter deposition and lift-off structuring as well as electroplating of metal layers used for the integrated temperature sensor (first metallization), as well as LED wiring (second metallization; thickness $1 \mu \mathrm{m}$ ) and contact pads (third metallization; thickness $5 \mu \mathrm{m}$ ), respectively.

The sequential integration of the LED chips onto the PI substrates employed flip-chip bonding which enabled the accurate and reliable assembly of individual LEDs to the electroplated bond pads by applying force, temperature and ultrasonic agitation. The $\mathrm{n}$ - and $\mathrm{p}$-pads of the LEDs are clearly visible through the transparent LED substrate, as indicated in Figs. 1E and F. Polymeric encapsulation layers were manually applied after the LED assembly (Supplementary Material and Fig. S2). The oCls received a polymeric underfill of the LEDs (epoxy EPO-TEK ${ }^{\circ}$ 301-2) and were encapsulated in a thin fluoropolymer film (Cytop ${ }^{\text {TM }}$ ) as well as in silicone, all applied by a manual dispensing process (Figs. 1E and F, and Fig. S2A) or using a precision-machined mold (Fig. 1H). The thicknesses of the fluoropolymer layer and underfill were in the low micrometer-range and defined by the height of the electroplated LED pads underneath the LEDs, while the silicone layer was about $50 \mu \mathrm{m}$ in thickness.

Functionality tests were performed before and after device passivation by sequentially addressing all LEDs (LED 1 - LED 10, Fig. 1G). In addition, motivated by the snail-like structure of the cochlea we tested the $\mathrm{oCl}$ mechanical functionality by inserting the optical implant into $300-\mu \mathrm{m}$-wide channels with different outer radii realized in polymethylmethacrylat (PMMA). We determined the minimal outer radius which differed depending on the LED pitch and the thickness of the silicone encapsulation. Figure $1 \mathrm{H}$ demonstrates the function of all ten LEDs of an oCl bent to an outer radius of $1 \mathrm{~mm}$. This oCl had an LED pitch of $350 \mu \mathrm{m}$ and was mold-encapsulated to an outer diameter of $300 \mu \mathrm{m}$. oCls with an LED pitch of $500 \mu \mathrm{m}$ and a silicone thickness of approximately $50 \mu \mathrm{m}$ could be bent to a radius of only $500 \mu \mathrm{m}$ while remaining functional.

\subsubsection{Electrical, optical and thermal characterization of the oCl}

The electrical and optical characterization of the oCls was performed by measuring the LED voltage $V$ (Fig. 2A) and radiant flux $\Phi$ (Fig. 2B) as a function of the LED current $I_{\text {LED }}$ which was varied between 0 
to $45 \mathrm{~mA}$ and pulsed at a duty cycle (DC) and a frequency of $10 \%$ and $10 \mathrm{kHz}$, respectively, resulting in pulses of duration $t_{p}=4 \mathrm{~ms}$. The threshold voltage of the applied LEDs was extracted from the data shown in Fig. $2 \mathrm{~A}$ to $V_{\mathrm{th}}=2.6 \mathrm{~V}$ which is comparable to the value given in the LED datasheet (23). The slopes of the I-V-curve are determined by the LED characteristics and the line resistance of the respective LED wiring, which likely explains the small differences among the various LEDs. On average a voltage of $4.32 \pm 0.04 \mathrm{~V}$ was measured at $I_{\mathrm{LED}}=30 \mathrm{~mA}$. The measurements of the optical radiant flux $\Phi$ as a function of $I_{\text {LED }}$ (Fig. 2B) were performed using a calibrated integrating sphere. The LEDs of this representative probe showed the same behavior and provided a time averaged radiant flux of $1.405 \pm 0.054 \mathrm{~mW}$ at $I_{\text {LED }}=30 \mathrm{~mA}(10 \% \mathrm{DC}$ and $10 \mathrm{kHz})$. This corresponds to a radiant flux of $14 \mathrm{~mW}$ during the $10-\mu \mathrm{s}$-long light pulses. Taking the LED foot print of $220 \times 270 \mu \mathrm{m}^{2}$ into account, $236 \mathrm{~mW} / \mathrm{mm}^{2}$ were emitted during those current pulses. Time averaged radiant emittances of 10,15 , and $20 \mathrm{~mW} / \mathrm{mm}^{2}$ relevant for optogenetic experiments (11) were consequently emitted at $I_{\text {LED }}=1.25$, 1.61, and $2.27 \mathrm{~mA}$, respectively. Spectral measurements of assembled and passivated LEDs showed light emission with an averaged center wavelength of $453.8 \pm 1.1 \mathrm{~nm}$.

Since the oCls are active devices with limited optoelectronic conversion efficiency, we set out to characterize heat production and dissipation using the integrated Pt meander (Fig. 1D) to determine the temperature increase of the probe as close as possible to an operating LED chip. Sensor calibration and the measurement setup are described in detail in the Supplementary Material. Based on a set of transient temperature measurements performed by applying different pulse durations $t_{\mathrm{p}}$ and LED currents /LED, the thermal time constant of the system was determined to be $11.8 \mathrm{~ms}$. The experiments were performed with the oCl sandwiched between layers of agarose gel kept at $37^{\circ} \mathrm{C}$. These conditions aimed to mimic the thermal properties of the perilymph fluid inside scala tympani of the cochlea. The experiments allowed to extract thermal relaxation functions modeling the temperature response of the probe to LED operation and to predict the maximum temperature increases for given LED currents and pulse durations. As expected, the modeled temperature evolution under the operated LED in

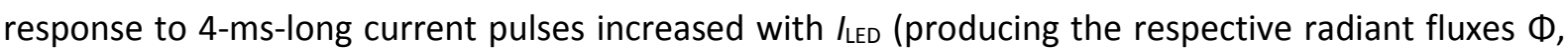
Fig. 2C). Correspondingly, for a constant /LED (38.9 mA, maximal /LED used in physiology resulting in $\Phi=18.1 \mathrm{~mW}$ ) the maximum temperature $T_{\max }$ rose with pulse durations $t_{\mathrm{p}}$ between 0 and $10 \mathrm{~ms}$ (Fig. 2D). Furthermore, Fig. $2 E$ shows $T_{\max }$ as a function of $\Phi$ for 4-ms-long pulses. Clearly, to operate the oCl at a given stimulation rate below a certain temperature, $I_{\text {LED }}$ and thus radiant flux or pulse duration should be limited. Since the temperature is measured within the $\mathrm{oCl}$ as close as possible to the heat source, i.e. the LED, it definitely overestimates the temperature increase in the surrounding tissue. Moreover, we note, that the heat dissipated by an oCl will be eliminated more efficiently within the scala tympani than by the agar gel of the experimental setup. We expect convection by the cochlear 
blood flow and conduction by structures neighboring scala tympani to dissipate the produced heat, to which the cochlear aqueduct likely also contributes.

A

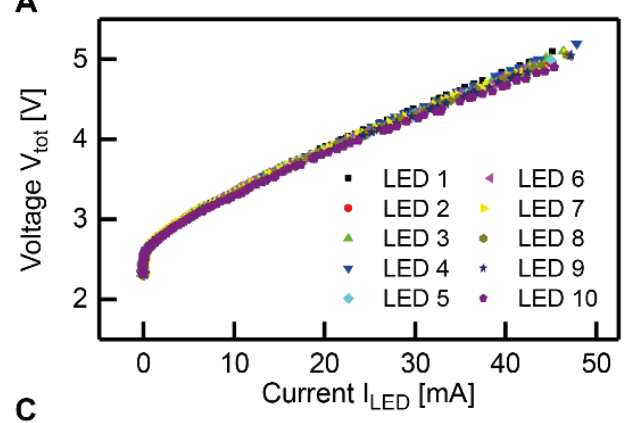

C

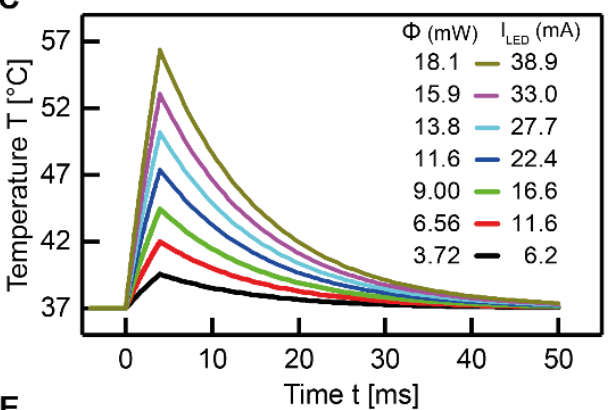

E

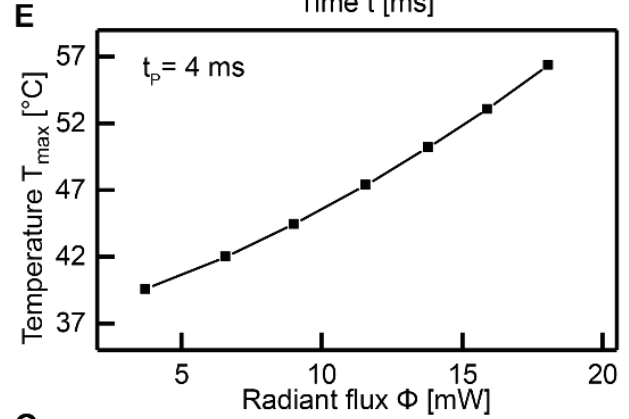

G

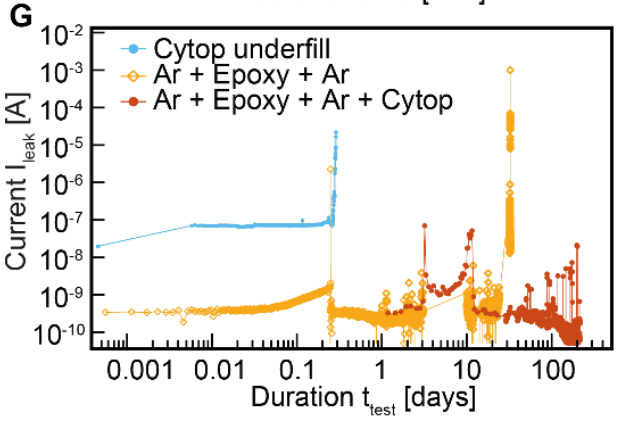

B

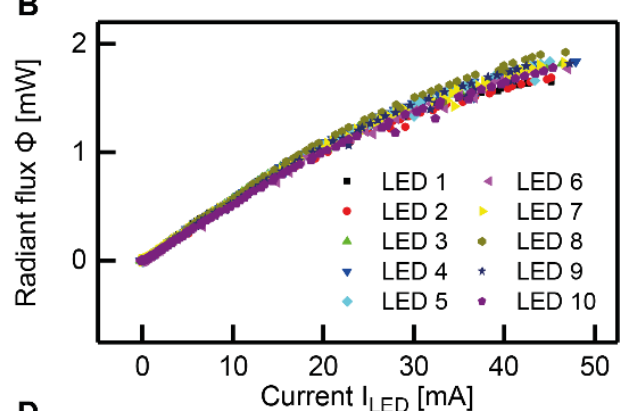

D

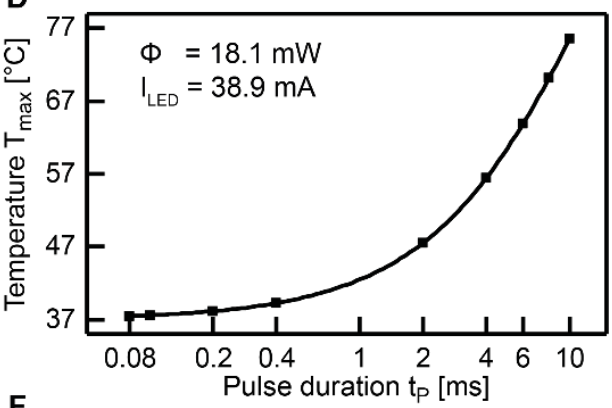

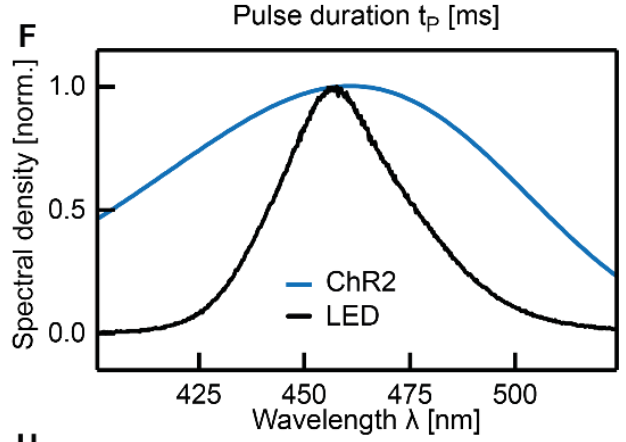

H

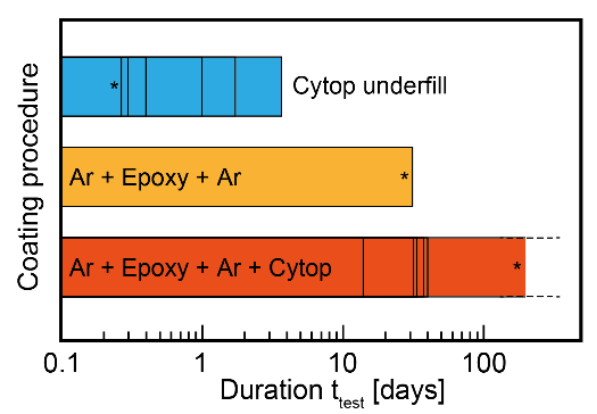

Figure 2. Electrical, optical and thermal characterization of an oCl.

(A) I-V curves of ten LEDs of an oCl exhibiting a threshold voltage of $2.6 \mathrm{~V}$. (B) Time-averaged radiant flux $\Phi$ of ten assembled and passivated LEDs of an oCl as a function of the LED current ILED applied at $10 \% \mathrm{DC}$ and a frequency of $10 \mathrm{kHz}$. (C) Modeled time-dependent temperature $\mathrm{T}$ of an oCl sample with

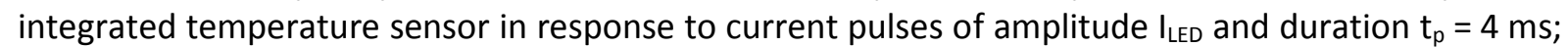
the pulses produced the indicated radiant flux values $\Phi$; the oCl was sandwiched in-between agarose gel layers kept at $37^{\circ} \mathrm{C}$. (D) Modeled maximum temperature $T_{\max }$ vs. $t_{p}$, when LED 1 was driven with $\mathrm{I}_{\text {LED } 1}=38.9 \mathrm{~mA}$ to $\Phi=18.1 \mathrm{~mW}$. (E) $\mathrm{T}_{\max }$ Vs. $\Phi$ at $t_{p}=4 \mathrm{~ms}$. (F) Spectral density distribution of LED 1 with peak emission at $457 \mathrm{~nm}$ (black line) and ChR2 normalized action spectrum (blue line) (24) with a peak at $460 \mathrm{~nm}$ (25). (G) Measured leakage currents $\mathrm{I}_{\text {leak }}$ vs. time of three representative oCl samples for various polymeric encapsulation procedures ( $\mathrm{Ar}=$ argon plasma treatment for surface activation), as detailed in the Supplementary Material and Fig. S2-A (up to stage vi). (H) oCl longevity derived from $\mathrm{I}_{\text {leak }}$ measurements, as shown in $(\mathrm{G})$; oCl failure is defined by $\mathrm{I}_{\text {leak }}>1 \mu \mathrm{A}$ for more ten consecutive 
measurement points. The temporal behavior of $l_{\text {leak }}$ of samples marked by an asterisk are shown in panel G; dashed horizontal lines indicate a long-term measurement still running; vertical lines in each horizontal bar indicate the failure of a specific sample.

\subsubsection{Longevity tests}

Longevity tests were performed on oCls which had been processed with different underfill and/or encapsulation materials. In saline solution, the integrated LEDs were reverse biased by a nonpermissive voltage of $-5 \mathrm{~V}$ between their $n$ - and $p$-lines measuring the leakage current $l_{\text {leak }}$ (for details see Supplementary Material). I leak values of three representative oCl samples show a clear dependence of longevity on the underfill and encapsulation procedure (Fig. 2G). The oCl indicated by blue symbols was underfilled using the solvent-containing fluoropolymer Cytop ${ }^{\mathrm{TM}}$ only and failed already after less than 8 hours as evident by the increasing $l_{\text {leak. }}$ In contrast, the oCl underfilled by solvent-free epoxy, with a surface activation by an argon (Ar) plasma before and after the underfill, survived for more than 30 days (orange). This indicates an advantage of using a solvent-free underfill, as further detailed in Figs. S2-C and S2-D. Finally, combining the repeated plasma treatment with epoxy underfill and Cytop $^{\mathrm{TM}}$ encapsulation (Fig. 2G, red symbols) further increased the longevity of the oCl to more than 200 days. These longevity results are summarized in Fig. $2 \mathrm{H}$ for the oCl samples characterized to date. We suggest, that the additional silicone encapsulation as used for the in vivo experiments, will support mechanical stability and further increase longevity as required for future chronic studies of multichannel optical stimulation.

\subsubsection{Optogenetic activation of the auditory pathway by multichannel oCls}

Next, we turned to transgenic ChR2-Venus rats (20) in order to test the feasibility of optogenetic activation of the auditory pathway by multichannel LED-based oCls. The oCls were connected via their ZIF connector interface (Fig. $3 \mathrm{~A}$ ) and driven in synchrony with the recordings of auditory brainstem responses. Prior to each experiment we measured the emitted light power per LED as a function of ILED with an integrating sphere and probed the electrical integrity of the oCls in saline solution. Then, using a retroauricular approach, we performed a posterior tympanotomy ("bullostomy", Fig. 3B), inserted the $\mathrm{oCl}$ probe through the round window and gently advanced it into the scala tympany. In few cases we inserted the oCl through an artificial small opening of the lateral wall of the middle cochlear turn (cochleostomy). The position of the implant was validated by $\mathrm{X}$-ray phase-contrast microscopy using a superbright laboratory X-ray source, as described before (22). With round window insertion and without forcing, the oCls usually covered the basal turn of scala tympani (Fig. 3C). We then compared supra-threshold auditory brainstem responses ( $A B R$ ) evoked by acoustic clicks (aABR, Fig. 3D, upper curve), 4-ms-long laser light pulses (oABR, Fig. 3D, middle curve, $473 \mathrm{~nm}, 6.6 \mathrm{~mW}$ delivered via a $200 \mu \mathrm{m}$ optical fiber inserted into the scala tympani via the round window) and oCl stimulation with 
the LED-based implant (OABR, front LED, $454 \mathrm{~nm}, 6.8 \mathrm{~mW}$, Fig. 3D, lower curve). The LED-stimulation caused an electrical onset artifact that was variable in size, preceded the oABR (asterisks mark the first positive peak, p1) and was followed by an artifact at the end of the stimulus. Except for these / LEDrelated artifacts, the OABRs elicited by laser and LED stimulation at comparable light intensities were similar. Both were also comparable in amplitude, but shorter in latency when compared to aABR, and showed less prominent later ABR peaks. The oABR strictly depended on the intrascalar position of the LED (Fig. 3E): when placed in the bulla (middle ear) no oABR could be elicited. Moreover, oABRs vanished after euthanizing the animal, despite intracochlear stimulation (Fig. 3E). We never observed OABRs in ChR2-negative rats (Figs. 3E and S3). Individual LEDs of the optical implant evoked slightly different $\mathrm{OABR}$ waveforms (Fig. 3F). While the oCl contained 10 LED chips, we were typically able to place only up to 5 of them inside the scala tympani with gentle insertion via the round window, resulting in stimulation of the basal turn. X-ray tomography indicated that this was likely because the oCl tip did not glide further due to contact with the lateral wall.

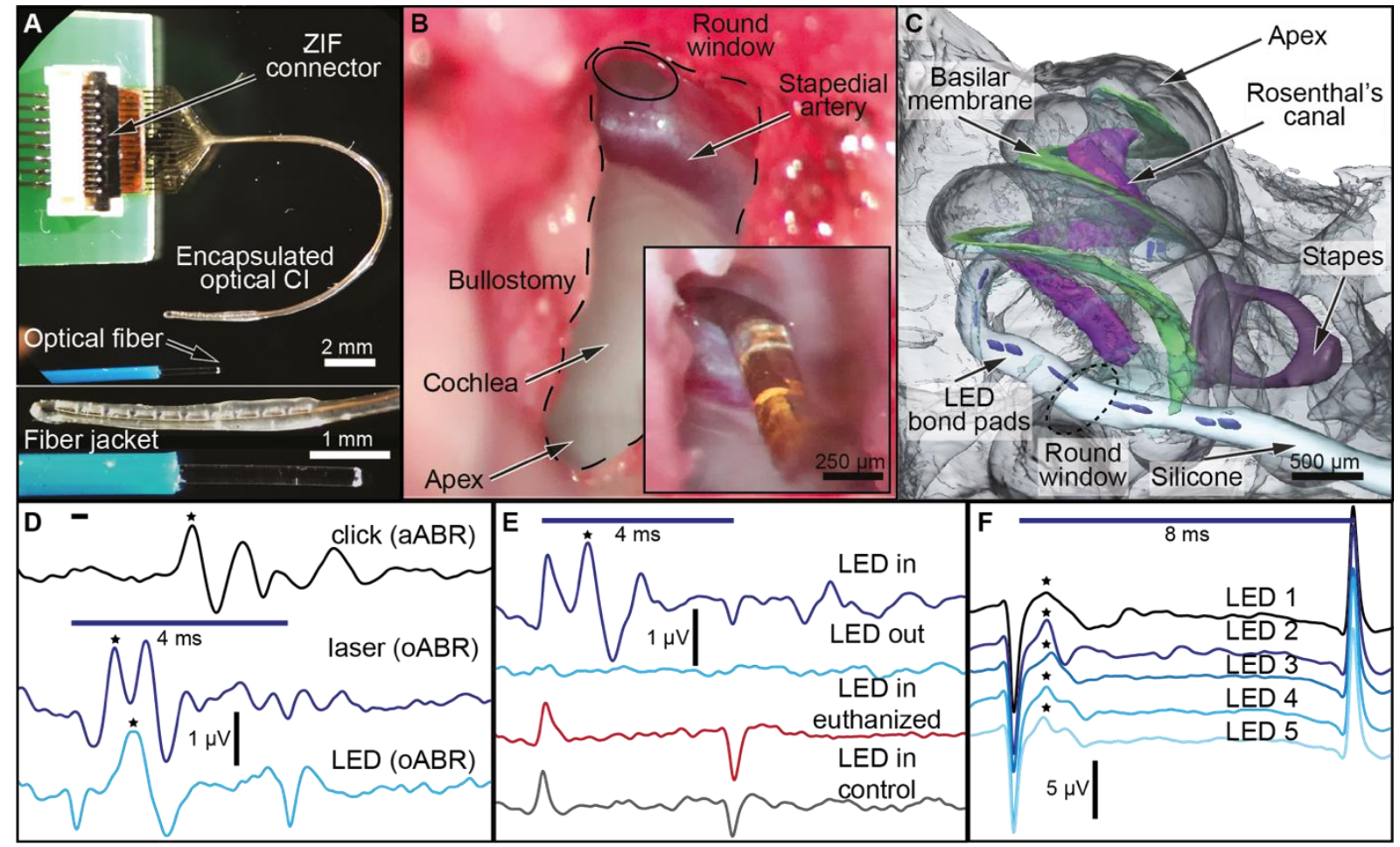

Figure 3. oCl stimulation of the auditory pathway in ChR2 transgenic rats.

(A) Silicone-encapsulated oCl connected to a PCB via a ZIF connector in comparison to a $200 \mu \mathrm{m}$ optical fiber, magnified in the lower panel. (B) Retroauricular approach to the middle ear: Surgical situs shows a bullostomy (dashed line) revealing the cochlea with the stapedial artery and round window. The inset shows the oCl inserted into the round window. (C) 3D model of an implanted rat cochlea derived from $X$-ray tomography showing the position of the LED-based oCl in the scala tympani when inserted via the round window. The insertion depth was $5.1 \mathrm{~mm}$ harboring 8 LEDs inside the scala tympani in this case. Note that the cochlea was reflected horizontally relative to position in (B) for easier orientation. (D) Comparison of aABR and OABRs evoked by laser light (473 nm) and LED light (454 nm). Top: average of acoustic responses to 500 clicks $(300 \mu \mathrm{s})$ at $60 \mathrm{~dB}(\mathrm{SPL})$ applied at $20 \mathrm{~Hz}$. Middle: rectangular 4-mslong laser stimulation at $6.8 \mathrm{~mW}$ and $10 \mathrm{~Hz}$ (average of 500 trials). Bottom: rectangular 4-ms-long LED 
stimulation at $6.6 \mathrm{~mW}$ and $10 \mathrm{~Hz}$ (average of 500 trials). First response peak (p1) is marked with an asterisk. (E) OABR recordings obtained from an LED stimulation (4-ms-long duration at $8.9 \mathrm{~mW}$ and $10 \mathrm{~Hz}, 500$ trials) with the LED placed inside the round window (dark blue), in the bulla outside the round window (light blue), the first LED at the tip of the array inside scala tympani after the animal was euthanized (red) and in a negative ChR2 rat (control, black): only onset and end artifacts are evident in both cases. (F) Representative oABRs obtained from five different LEDs (8-ms-long duration at $2.15 \mathrm{~mW}$ and $2 \mathrm{~Hz}, 300$ trials) of an implanted oCl.

Next, we performed a first characterization of the reproducibility and reliability of the multichannel oCls. First, we compared the oABRs elicited by LED 1 of five different oCls inserted into the scala tympani of the same rat via cochleostomy for most cautious insertion (Fig. 4A). The p1-morphology of the oABRs was qualitatively conserved with some variability of amplitude and latency (Fig. 4A-B). The electrical artifacts varied among oCl insertions. The p1-amplitude increased more than one order of magnitude as a function of light power. At high radiant flux (18 mW), stimuli as short as $100 \mu \mathrm{s}$ were sufficient to elicit OABRs, which grew in amplitude with duration up to $2 \mathrm{~ms}$ and then leveled off (Fig. 4C). The p1-amplitude decreased with increasing stimulus rate, but remained detectable up to $200 \mathrm{~Hz}$ (Fig. 4D).

Next, we compared oABRs evoked by laser light (optical fiber implanted via cochleostomy) to those driven by an oCl (implanted via cochleostomy) in 5 different animals (Figs. 4E-H). We first studied the response to the laser and then inserted $\mathrm{oCl} 2$ (which generated small electrical artifacts). We observed significant variability in oABRs to LED-1-stimulation among the animals. The oABRs evoked by the oCI were comparable in amplitude to those elicited by laser pulses of the same radiant flux, pulse duration and rate (Fig. 4F). The dependence of the oCl-evoked oABRs on pulse duration was comparable to the result in Fig. 4C. For the laser stimuli with their higher radiant flux, we observed a tendency of the oABRs to decline beyond durations of $2 \mathrm{~ms}$ (Fig. 4G). The rate-dependent p1-amplitude decline seemed shallower for LED-stimulation than for laser stimulation (Fig. 4H).

Finally, we performed first multichannel stimulation experiments aiming to study potential additive effects of LEDs recruited in addition to LED 1 . The reasoning was that, if, indeed, each LED only activated a sub-population of the spiral ganglion, the $O A B R$ amplitude should grow when recruiting additional, more basal LEDs that would activate further SGNs. Figure S4 shows an exemplary experiment in which we subsequently recruited additional LEDs and studied the effects on the oABR amplitude for different radiant flux, pulse durations and stimulation rates. Additivity was strongest when activating LED 2 in addition to LED 1 and lower gain in amplitude was found when recruiting further LEDs. Additivity of stimulation by multiple LEDs indicates that the spread of excitation from the LEDs is limited at least at a low stimulus intensity such that the more basal LEDs could drive further SGNs. 
A
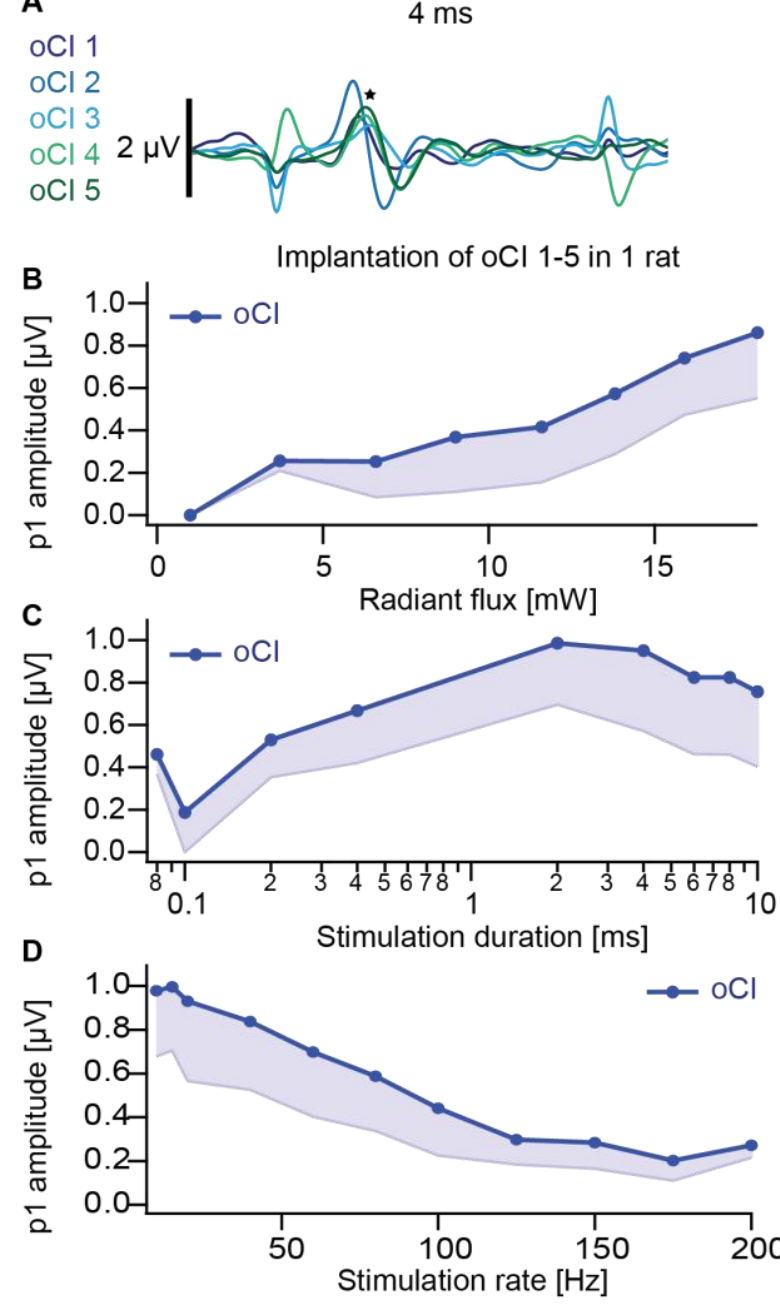

E
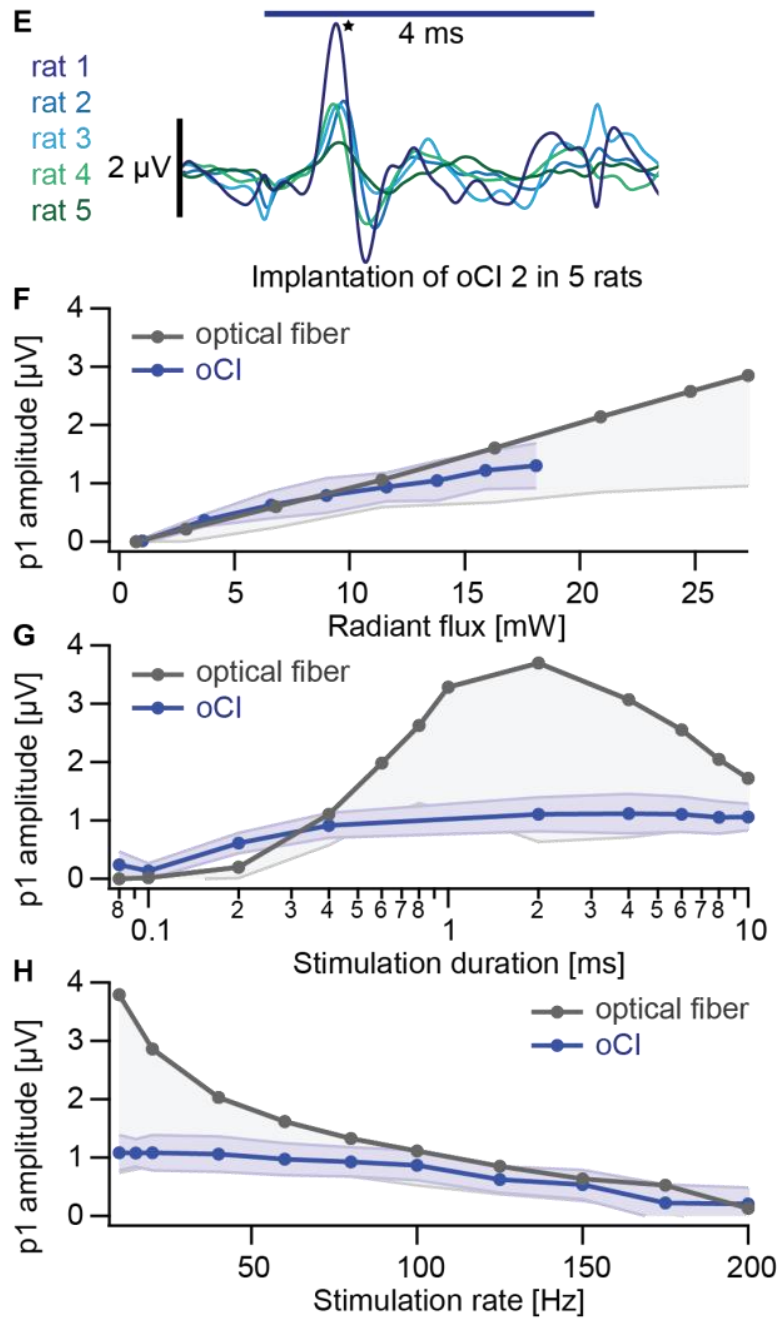

Figure 4. Characterizing $\mathrm{OCl}$ stimulation by recordings of $\mathrm{OABRs}$ in rats.

Optical $\mathrm{Cl}$ or optical fiber were placed on the middle cochlear turn via a cochleostomy. (A) Exemplary oABRs driven by LED 1 of oCls 1-5 (color code) in the transgenic ChR2 rat used in B - D with a radiant flux of $18.1 \mathrm{~mW}$ (4-ms-long stimulus at $10 \mathrm{~Hz}, 500$ trials throughout the figure). Note the onset and end artifacts of opposite polarity that flank the stimulus. First peak of response ( $p 1$ ) is marked with an asterisk. (B - D) OABR amplitude of p1 recorded from one rat with 5 different oCls as a function of radiant flux (B, duration: $4 \mathrm{~ms}$, rate: $10 \mathrm{~Hz}$ ), stimulus duration (C, radiant flux $18.1 \mathrm{~mW}$, rate: $10 \mathrm{~Hz}$ ) and stimulation rate (D, $18.1 \mathrm{~mW}$, duration: $2 \mathrm{~ms}$ ). Negative standard deviation (SD) shown in light blue. (E) Exemplary oABRs from transgenic ChR2 rats 1-5 (color code) used in ( $\mathbf{F}-\mathbf{H}$ ) stimulated by LED 1 of oCl 2. (F-H) p1 amplitude of oABRs recorded from four rats, first using a $200 \mu \mathrm{m}$ optical fiber (coupling in the light of a $473 \mathrm{~nm}$ laser; dark grey symbols), thereafter employing LED 1 of oCl 2 (blue symbols), presented as a function of radiant flux (F, duration: $4 \mathrm{~ms}$, rate: $10 \mathrm{~Hz})$, stimulus duration (G, radiant flux: $18.1 \mathrm{~mW}(\mathrm{oCl}), 27.3 \mathrm{~mW}$ (laser), rate: $10 \mathrm{~Hz}$ ) and stimulation rate $(\mathbf{H}$, radiant flux $18.1 \mathrm{~mW}$ (oCl), $27.3 \mathrm{~mW}$ (laser), duration: $2 \mathrm{~ms}$ ). The oCl SDs are shown in light blue, laser SDs (only negative SDs shown) in light grey. 


\subsection{Discussion}

Here we demonstrate the technological fabrication and biological application of LED-based multichannel optical cochlear implants. The design and fabrication of these oCls was guided by the diameter of scala tympani of the rat cochlea $(>300 \mu \mathrm{m})$, as estimated from X-ray tomography, and the minimal bending radius to comply with $(1 \mathrm{~mm})$. This obviously placed constraints on the design of oCls for rodents, also restricting the number of integrated LED chips, which in our case were individually addressed as is the case of electrodes in current clinical Cls. As the applied LED chips require currents in the mA-range for light emission that activates optogenetically-modified SGNs, line width and height were requested to be as large as possible. The LED dimensions of $270 \times 220 \times 50 \mu \mathrm{m}^{3}$ were small enough for an implantation into the rat cochlea and large enough for LED assembly using flip-chip bonding. The LED size and pitch turned out to limit the bending radii of the oCl. Reducing probe dimensions and bending radii can be achieved by the integration of thin-film $\mu$ LEDs, as recently demonstrated (18). The optical characterization of the radiant flux indicated that a sufficient optical power can be generated for optogenetic activation of SGNs, which was directly revealed by biological experiments. The temperature modeling based on measurements with passivated oCls in contact with agarose gel enabled the determination of the implant temperature in a worst-case scenario without any cooling by the cochlear blood flow. For radiant emittances of $15 \mathrm{~mW} / \mathrm{mm}^{2}$ at the LED surface we extracted a temperature increase of the integrated temperature sensor under an operated LED of approximately $2 \mathrm{~K}$. This seems acceptable according to the ISO 14708-1 standard for implantable medical devices limiting the temperature increase to $2 \mathrm{~K}$. Future in vivo temperature measurements using the integrated Pt meander will help to set the upper limits for operating oCls in the cochlea without a risk of overheating the surrounding tissue.

The novel oCls could readily be inserted into the scala tympani of the rat via the round window or cochleostomy and were capable of eliciting OABRs comparable to those evoked by laser-pulses but flanked by electrical artifacts. Since no leakage currents were observed, we assume that the artifacts were of capacitive origin, which calls for a better electrical shielding of the oCl supply lines. The oClelicited OABRs were highly specific as they were absent when (i) the stimulating LED chip was outside the scala tympani, (ii) the animal was euthanized, and (iii) wild-type rats lacking ChR2-expression in SGNs were stimulated. Sequential insertion of several oCls into a given rat cochlea as well as the subsequent insertion of one $\mathrm{oCl}$ in several rats was possible and compatible with function, indicating general utility as well as sufficient mechanical and electrical stability of the oCl prototypes. While some variability of oABRs was found with both experiments, it seemed less pronounced than in our previous experiments where oABRs were vastly different in response to the focused light of a power-LED (11). Importantly, maximal OABR amplitudes, thought to relate to the number of SGNs that were synchronously activated by the LED, were in the low $\mu \mathrm{V}$ range, quite similar to $\mathrm{AABR}$, but much smaller 
than what we had previously reported for oABRs for mice (11). This suggests better specificity of activation of the auditory pathway by oCls in the present study. The observed OABR amplitudes grew over more than one order of magnitude of radiant flux and did not show evidence for saturation, which indicates a large dynamic range of optical coding (more than $20 \mathrm{~dB}$, typically less than $10 \mathrm{~dB}$ in electrical $\mathrm{Cl}(6))$. Unlike in our previous study, where oABRs failed even below $100 \mathrm{~Hz}$ (11), we could demonstrate oABRs elicited by oCls in ChR2-transgenic rats for stimulus rates as high as $200 \mathrm{~Hz}$. While we cannot fully explain this discrepancy, we speculate that it, too, might relate to more specific activation of the auditory pathway in the present study.

Much remains to be done in order to develop multichannel oCls as tools for research and for future translation into the clinic. As an example, long-term in vitro testing of mechanical and optoelectronic integrity as well as chronic implantation studies of reliability and safety will be required. Moreover, while our study provides a basic proof of functionality for a few LEDs, future studies will aim at establishing and characterizing arrays with a higher emitter density. Thin-film GaN $\mu$ LEDs are well established and can reach power efficiencies of up to $50 \%$ (25). Hence, they are good candidates as emitters for 'active' blue oCls (12). However, as Lambertian emitters they are not ideal for providing highly focused optical stimulation. Focusing by micro-lenses (27) and/or 'modiolus-hugging' (28) in order to bring the $\mathrm{oCl}$ as close as possible to the medial wall of the cochlea and to let the LEDs directly face the SGN somas in Rosenthal's canal can be expected to reduce the spread of light. However, even without optimization LED-stimulation might realize some spatial selectivity. In our study, a first test of simultaneous multichannel optical stimulation using LED-based oCls showed an increase of oABR amplitude at least upon the recruitment of one additional LED. We suggest that this reflects independence of both LEDs regarding SGN activation to some extent, as it likely happened at different tonotopic positions. Future characterization of the spread of optogenetic excitation by multichannel $\mu L E D$ oCls will require recordings from the auditory midbrain, where the central nucleus of the inferior colliculus provides convenient access to the tonotopic map (10, 26-28). 


\subsection{Materials and methods}

See supplemental information.

\subsection{References and notes}

1. WHO | Deafness and hearing loss. WHO, (available at http://www.who.int/mediacentre/factsheets/fs300/en/).

2. T. Moser, Sci. Transl. Med., in press, doi:10.1126/scitranslmed.aac7545.

3. H. Fukui, Y. Raphael, Gene therapy for the inner ear. Hear. Res. 297, 99-105 (2013).

4. J. V. Brigande, S. Heller, Quo vadis, hair cell regeneration? Nat. Neurosci. 12, 679-685 (2009).

5. H. Fastl, E. Zwicker, Psychoacoustics: facts and models (Springer-Verlag New York Inc., 2007).

6. F.-G. Zeng et al., Cochlear implants: system design, integration, and evaluation. IEEE Rev. Biomed. Eng. 1, 115-142 (2008).

7. A. Kral, R. Hartmann, D. Mortazavi, R. Klinke, Spatial resolution of cochlear implants: The electrical field and excitation of auditory afferents. Hear. Res. 121, 11-28 (1998).

8. R. V. Shannon, Multichannel electrical stimulation of the auditory nerve in man. II. Channel interaction. Hear. Res. 12, 1-16 (1983).

9. L. M. Friesen, R. V. Shannon, D. Baskent, X. Wang, Speech recognition in noise as a function of the number of spectral channels: Comparison of acoustic hearing and cochlear implants. J. Acoust. Soc. Am. 110, 1150 (2001).

10. K. E. Fishman, R. V. Shannon, W. H. Slattery, Speech recognition as a function of the number of electrodes used in the SPEAK cochlear implant speech processor. J. Speech Lang. Hear. Res. JSLHR. 40, 1201-1215 (1997).

11. V. H. Hernandez et al., Optogenetic stimulation of the auditory pathway. J. Clin. Invest. 124, 1114-1129 (2014).

12. M. Jeschke, T. Moser, Considering optogenetic stimulation for cochlear implants. Hear. Res. 322, 224-234 (2015).

13. A. E. Hight et al., Superior temporal resolution of Chronos versus channelrhodopsin-2 in an optogenetic model of the auditory brainstem implant. Hear. Res. 322, 235-241 (2015).

14. W. Guo et al., Hearing the light: neural and perceptual encoding of optogenetic stimulation in the central auditory pathway. Sci. Rep. 5, 10319 (2015).

15. B. Fan, W. Li, Miniaturized optogenetic neural implants: a review. Lab Chip. 15, 3838-3855 (2015).

16. M. T. Alt et al., Let There Be Light - Optoprobes for Neural Implants. Proc. IEEE. 105, 101-138 (2017). 
17. T. Kim et al., Injectable, cellular-scale optoelectronics with applications for wireless optogenetics. Science. 340, 211-216 (2013).

18. C. Goßler et al., GaN-based micro-LED arrays on flexible substrates for optical cochlear implants. J. Phys. Appl. Phys. 47, 205401 (2014).

19. R.-H. Kim et al., Waterproof AllnGaP optoelectronics on stretchable substrates with applications in biomedicine and robotics. Nat. Mater. 9, 929-937 (2010).

20. H. Tomita et al., Visual Properties of Transgenic Rats Harboring the Channelrhodopsin-2 Gene Regulated by the Thy-1.2 Promoter. PLoS ONE. 4, e7679 (2009).

21. M. Schwaerzle, J. Nehlich, S. Ayub, O. Paul, P. Ruther, in Technical Digest IEEE 29th International Conference on Micro Electro Mechanical Systems (MEMS) (2016), pp. 395-398.

22. M. Bartels, V. H. Hernandez, M. Krenkel, T. Moser, T. Salditt, Phase contrast tomography of the mouse cochlea at microfocus x-ray sources. Appl. Phys. Lett. 103, 083703 (2013).

23. Cree Inc., “Cree ${ }^{\circledR}$ TR2227 ${ }^{\mathrm{TM}}$ LEDs, C460TR2227-S2100” (Durham, 2016).

24. F. Schneider, C. Grimm, P. Hegemann, Biophysics of Channelrhodopsin. Annu. Rev. Biophys. 44, 167-186 (2015).

25. G. Nagel et al., Channelrhodopsin-2, a directly light-gated cation-selective membrane channel. Proc. Natl. Acad. Sci. U. S. A. 100, 13940-5 (2003).

26. A. Laubsch, M. Sabathil, J. Baur, M. Peter, B. Hahn, High-power and high-efficiency InGaNbased light emitters. Electron Devices IEEE Trans. On. 57, 79-87 (2010).

27. H. W. Choi, E. Gu, C. Liu, J. M. Girkin, M. D. Dawson, Fabrication and evaluation of GaN negative and bifocal microlenses. J. Appl. Phys. 97, 063101 (2005).

28. S. M. Cords et al., A silastic positioner for a modiolus-hugging position of intracochlear electrodes: electrophysiologic effects. Am. J. Otol. 21, 212-217 (2000).

29. I. Stiebler, G. Ehret, Inferior colliculus of the house mouse. I. A quantitative study of tonotopic organization, frequency representation, and tone-threshold distribution. J. Comp. Neurol. 238, 65-76 (1985).

30. R. L. Snyder, J. C. Middlebrooks, B. H. Bonham, Cochlear implant electrode configuration effects on activation threshold and tonotopic selectivity. Hear. Res. 235, 23-38 (2008).

31. D. M. Harris, Current source density analysis of frequency coding in the inferior colliculus. Hear. Res. 25, 257-266 (1987).

32. W. van Aarle et al., The ASTRA Toolbox: A platform for advanced algorithm development in electron tomography. Ultramicroscopy. 157, 35-47 (2015).

33. W. van Aarle et al., Fast and flexible X-ray tomography using the ASTRA toolbox. Opt. Express. 24, 25129-25147 (2016). 


\subsection{Acknowledgments}

The research leading to these results has received funding from the German Research Foundation (DFG) through the Cluster of Excellence BrainLinks-BrainTools (grant no. EXC 1086, P.R.), the Collaborative Research Center 889 (C.W.), DFG Research Center and Cluster of Excellence, Center for Nanoscale Microscopy and Molecular Physiology of the Brain (EXC171/FZT-103, T.M.) and the Leibniz program (T.M.), from the Federal Ministry of Education and Research (BMBF) via the project Optical Cochlear Implant (Optical_Cl, grant no. 13N13728, T.M. and P.R.) as well from the European Research Council (ERC Advanced Grant "OptoHear" to T.M.). The authors gratefully acknowledge Michael Reichel and Armin Baur (both IMTEK-RSC) for support during the cleanroom fabrication, Mareike Töpperwien and Tim Salditt for letting us use their X-ray microscope and for advice, Peter Wenig for electronic design, and Rainer Schürkötter (MPI for Biophysical Chemistry, Göttingen) for silicone encapsulation of a subset of probes.

Competing financial interests: The authors declare no competing financial interests.

\subsection{Supplementary information}

\section{Supplementary materials and methods}

Supplementary Fig. S1. Fabrication of the highly flexible oCl substrates.

Supplementary Fig. S2. Assembly and passivation of oCls.

Supplementary Fig. S3. oCls do not evoke potentials in littermate control rats lacking ChR2 or in dead ChR2-positive rats.

Supplementary Fig. S4. Additivity of LEDs in eliciting oABRs.

Supplementary Movie S1. Visualization of implanted cochlea by high resolution X-ray tomography.

3D-visualization of an explanted rat cochlea obtained by $\mathrm{X}$-ray phase contrast tomography first displayed as raw X-ray sections and volume rendering representing pixel values in grey scale. Segmented model reveals LEDs (blue), basilar membrane (green) scala tympani (turquois) and Rosenthal's canal (purple). Towards the end the camera follows the path of the oCl into the scala tympani via the round window.

Supplementary Movie S2. Visualization of oCl stimulation in the explanted rat cochlea.

Explanted rat cochlea submerged in thiodiethanol (to render the bone translucent) with fully implanted $10 \mathrm{LED}$ oCl in the basal turn via the round window. LEDs were activated via fast Fourier transformed audio track signal. 


\subsection{Supplementary materials and methods}

\subsubsection{Microfabrication of the optical cochlear implant substrates}

Figure S1 summarizes the microfabrication of the highly flexible triple layer polyimide (PI) substrates. Their total thickness of $15 \mu \mathrm{m}$ results from three layers of PI and three metal layers. A first 5 - $\mu \mathrm{m}$-thick PI (UPIA-ST, UBE Europe GmbH, Duesseldorf, Germany) layer was spin-coated onto a silicon (Si) handle wafer and cured at $450^{\circ} \mathrm{C}$ for $3 \mathrm{~h}$ (Fig. S1-A). This was followed by the first metallization (platinum (Pt), $250 \mathrm{~nm}$ ) which was sputter-deposited after applying an oxygen plasma activation of the PI surface at $80 \mathrm{~W}$ for $1 \mathrm{~min}$ (Fig. S1-A). This first metal layer was lift-off patterned using an image reversal photoresist (AZ5214E, Merck KGaA, Darmstadt, Germany). The second PI layer was spin-coated to a thickness of $2.5 \mu \mathrm{m}$ and patterned using a photoresist mask (AZ9260, Merck KGaA) and reactive ion etching (RIE), as shown in Fig. S1-B. To improve the adhesion of this second PI layer to the PI substrate an oxygen plasma ( $80 \mathrm{~W}, 1 \mathrm{~min}$ ) was again applied. The second metallization comprising the LED supply lines made use of a sputter-deposited seed layer (Pt/gold (Au)/titanium (Ti), 30/200/30 nm). The seed layer adhesion was improved by an argon (Ar) plasma treatment directly prior to the metal deposition. This was followed by deposition and patterning of a second photoresist mask, the removal of the upper 30-nm-thick Ti adhesion layer using $1 \%$ hydrofluoric acid (HF), and electroplating of Au to a thickness of $1 \mu \mathrm{m}$ for the LED wiring (Fig. S1-C). After removal of the electroplating mask, the remaining Ti and $\mathrm{Au}$ seed layers around the electroplated structures were wet etched using $1 \% \mathrm{HF}$ and potassium iodide/iodine $\left(\mathrm{KI} / \mathrm{I}_{2}\right)$ solution, respectively, while the Pt layer was dry etched using an Ar plasma (Fig. S1-D). For the third PI layer with a thickness of $1 \mu \mathrm{m}$ and the third metallization of the LED bond pads with a thickness of $5 \mu \mathrm{m}$ we applied the same procedure as for the second layer level (Fig. S1-EG). The PI and thus probe patterning employed RIE using a 30- $\mu$ m-thick AZ9260 photoresist as the masking layer (see Fig. S1-H). Finally, the oCl substrates could be peeled off the wafer using tweezers (Fig. S1-I).

\subsubsection{Assembly and packaging}

oCl substrates (see Fig. S2-A(i)) peeled off the handle wafer were individually turned into functional oCls starting with the LED chip assembly (see Fig. S-2A(ii)). Ten LED chips (Cree C460TR2227-S2100, Three Five (III-V) Materials, Inc., NY, USA) were sequentially flip-chip bonded with their $\mathrm{n}$ - and $\mathrm{p}$ contacts on the corresponding pads on the PI substrate. The flip-chip bonder (Fineplacer $96 \lambda$, Finetech $\mathrm{GmbH}$, Berlin, Germany) offers an accuracy of $\pm 5 \mu \mathrm{m}$; we applied a bonding force of $1.5 \mathrm{~N}$ at a temperature of $120^{\circ} \mathrm{C}$ and an ultrasonic energy of $300 \mathrm{~mW}$. These process parameters ensure the mechanical and electrical interconnection of the LED bond pads to the 5 - $\mu \mathrm{m}$-high substrate pads. After LED assembly, we rinsed the oCls with DI water before an Ar plasma (50 W, $1 \mathrm{~min}$ ) cleaned and 
activated the PI surface (Fig. S2-A(iii)). Immediately thereafter, the LEDs were underfilled with a solvent free epoxy (EPO-TEK ${ }^{\circ}$ 301-2, John P. Kummer GmbH) (Fig. S2-A(iv)). The underfill was cured for 12 hours in an oven at $120^{\circ} \mathrm{C}$. A first polymeric encapsulation using Cytop ${ }^{\mathrm{TM}} 809 \mathrm{M}$ (Asahi Glass Co. Ltd., Tokyo, Japan) was applied immediately after a further plasma treatment (Ar, $50 \mathrm{~W}, 1 \mathrm{~min}$ ), as shown in Figs. S2-A(v) and A(vi). A soft bake of this passivation was performed in an oven at $50^{\circ} \mathrm{C}$ for $30 \mathrm{~min}$ and $80^{\circ} \mathrm{C}$ for $1 \mathrm{~h}$. This stage of fabrication including an additional hard bake step at $180^{\circ} \mathrm{C}$ for $1 \mathrm{~h}$ was used for the longevity testing (Fig. 2G, H). For the oCl samples used in vivo, an oxygen plasma (50 W, $1 \mathrm{~min}$ ) was then applied (Fig. S2-A(vii)) to improve the layer adhesion of the final silicone (ELASTOSIL ${ }^{\circ}$ RT 604, Wacker Chemie AG, Muenchen, Germany) passivation layer (see Fig. S2-A(iii)). The final annealing process was performed in two steps at 80 and $180^{\circ} \mathrm{C}$, both applied for $1 \mathrm{~h}$ each.

The top view and cross-sectional schematics in Fig. S2-B illustrate the layered structure of the oCl and the used materials in the LED area. Figures S2-C and D demonstrate the benefit of using a solvent-free underfill: when Cytop ${ }^{\mathrm{TM}}$ was used as underfill gas bubbles were observed underneath the LEDs (Fig. S2D), while the use of the solvent-free EPO-TEK ${ }^{\circ}$ 301-2 avoided bubble formation (Fig. S2-D).

\subsubsection{Experimental characterization}

The optoelectronic characterization of the oCls was performed with a custom-made setup consisting mainly of a current source and an optical measurement system (OMS). The current source was built using a diode driver (iC-HKB, iC-Haus $\mathrm{GmbH}$, Bodenheim, Germany) combined with an evaluation board from iC-Haus GmbH. The DAQ PCle-6259 board (National Instruments, Austin, USA) was controlled by a LabView program. The LED current supplied by the driver and the voltage-drop over the oCl were measured and stored via the LabView program. In parallel, the program controls the OMS consisting of an integrating sphere (ISP-50-I-USB, Ocean Optics, Ostfildern, Germany) combined with a spectrometer (USB4000, Ocean Optics). It enables the radiant flux $\Phi$ as well as the spectral distribution of the emitted light to be determined. For this purpose, the LED-carrying tip of the oCI was inserted into the 8-mm-wide aperture of the integrating sphere while varying the LED current /LED from 0 to $45 \mathrm{~mA}$ at a DC of $10 \%$ and a frequency of $10 \mathrm{kHz}$ for the time-averaged radiant flux measurements. The thermal characterization was performed using the integrated Pt meander as a temperature sensing element. In a first calibration measurement, we extracted the resistance change of the Pt meander as a function of temperature between $22.5^{\circ} \mathrm{C}$ and $58.5^{\circ} \mathrm{C}$. The resistance change was measured using a four-wire setup by applying a constant Pt meander current of $100 \mu \mathrm{A}$ and measuring the voltage-drop along the Pt meander. The extracted temperature coefficient of resistance was $2.71 \times 10^{-3} \mathrm{~K}^{-1}$ at $37^{\circ} \mathrm{C}$. In order to determine the temperature increase of an LED in operation, the oCl was sandwiched between two agarose gel slides kept at $37^{\circ} \mathrm{C}$ and simulating the thermal coupling of the probe to tissue. Measurements of the meander resistance and thus temperature as a function of 
time were performed while applying LED current pulses of various magnitudes $I_{\text {LED }}$ and durations $t_{\mathrm{p}}$. For each combination of $I_{\text {LED }}$ and $t_{\mathrm{p}}, 16$ measurements were averaged. By fitting exponential relaxation functions to the data, a thermal time constant of $11.8 \mathrm{~ms}$ was extracted. As a second relevant parameter, the asymptotic temperature increase of the Pt meander under the LED was determined as well. The thermal model of the $\mathrm{oCl}$ allows predicting the maximal temperature increase for a given LED current, pulse duration and stimulation frequency.

\subsubsection{Passivation validation}

The characterization of the $\mathrm{oCl}$ encapsulation was performed with an automated leakage current test setup. Here, the oCl was connected via a ZIF connector to a printed circuit board (PCB) integrated into a tailored silicone plug of a test tube. The oCl was inserted into the test tube containing Ringer's solution simulating the environment of the probe in the cochlea. The leakage test was performed by applying the maximum recommended LED reverse bias voltage of $-5 \mathrm{~V}$ while measuring the leakage current $l_{\text {leak }}$ between the $n$ - and $p$-lines of the LED using a source meter (Model 2602A, Keithley Instruments, USA). The source meter was controlled via a GPIB interface using a LabView program storing the current value at predefined time intervals. The measurement was terminated once $l_{\text {leak }}$ exceeded the set limit of $1.0 \mu \mathrm{A}$ for 10 consecutive measurement points of $/$ leak.

\subsubsection{Animals}

Experiments were performed on adult wild-type and transgenic channelrhodopsin- 2 rats of either sex (Thy1-ChR2-Venus, (20)). All experiments conformed to the local and national guidelines for the care and use of laboratory animals in research and were approved by the local authorities of the State of Lower Saxony (LAVES).

\subsubsection{Surgery}

For in vivo experiments transgenic rats were anesthetized with isoflurane $(4 \%$ at $1 \mathrm{l} / \mathrm{min}$ for induction, $1-2 \%$ at $0.4 \mathrm{l} / \mathrm{min}$ for maintenance) and appropriate analgesia was obtained with subdermal injection of buprenorphine. The animals were then placed onto a custom-designed heating blanket $\left(37^{\circ} \mathrm{C}\right)$ on a vibration isolation table in a sound-proof chamber. Access to the left cochlea for stimulation was achieved by a retroauricular approach. Optical stimulation was performed through the round window or cochleostomy. For morphological studies we euthanized the animals with isoflurane and then explanted and fixed the cochlea. 


\subsubsection{ABR recordings}

oCls or a 200- $\mu$ m optical fiber coupled to a blue laser (473 nm, MLL-FN-473-100, $100 \mathrm{~mW}$ DPSS; Changchun New Industry Optoelectronics) were inserted into the cochlea via the round window (or via a cochleostomy, where indicated). The radiant flux of laser and oCls were calibrated with a laser power meter (Gentec-EO Solo 2, München, Germany) and a custom built integrating sphere, respectively. The LED-based optical probes were controlled via digital-to-analog (DA) outputs achieving pulse sequences with a resolution of $2 \mu$ s driven with an operational amplifier. The light output was defined by the voltage output of the the DA. ABRs were recorded by needle electrodes underneath the pinna, on the vertex, and a reference electrode on the neck. The differential potential between vertex and mastoid subdermal needles was amplified using a custom-designed amplifier (gain 10,000), sampled at a rate of $50 \mathrm{kHz}$ (NI PCl-6229, National Instruments), and filtered off-line (0.3 kHz to $3 \mathrm{kHz}$ Butterworth filter) for acoustically and optically evoked ABRs.

Stimulus generation and presentation (acoustic and optic), and data acquisition were realized by a customized software in MATLAB (The MathWorks, Inc.) employing National Instruments cards in combination with custom-built hardware to amplify and attenuate audio signals. In case of acoustically evoked ABRs, clicks of $0.3 \mathrm{~ms}$ length were presented in an open near field via a single loudspeaker (Vifa, Avisoft Bioacoustics) placed on average $30 \mathrm{~cm}$ in front of the animal at the level of the animal's head. Sound pressure levels were calibrated with a 0.25-inch microphone (D 4039, Brüel \& Kjaer $\mathrm{GmbH}$ ) and measurement amplifier combination (2610; Brüel \& Kjaer GmbH).

\subsubsection{X-ray tomography}

Positioning of the $\mathrm{oCl}$ was confirmed with phase-contrast tomography at a compact laboratory X-ray source essentially as previously described (22). The custom-built cone-beam in-line tomography instrument equipped with a liquid-metal X-ray source (JXS D2, Excillum, Sweden) operated at $70 \mathrm{kV}$ acceleration voltage was combined with a $20 \mu \mathrm{m}$ LuAG-scintillator-based fiber-coupled CCD detector (6.5 $\mu \mathrm{m}$ pixel size; C12849-102U; Hamamatsu). Fast Fourier-based phase reconstruction was used to calculate the image volume. Reconstruction was performed using the ASTRA toolbox $(29,30)$. Segmentation and 3D visualization of the reconstructed volume was performed with Avizo 3D 9 (Visualization Sciences Group, FEI Company). Automatic histogram-based segmentation was used to visualize bony structures. The basilar membrane, Rosenthal's canal, scala tympani were traced with semiautomatic segmentation. Insertion depth was measured with a fitted spline curve to markers along the oCl. 
A

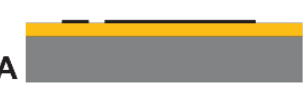

D
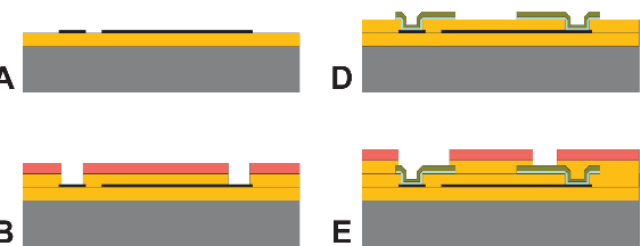

$\mathbf{E}$
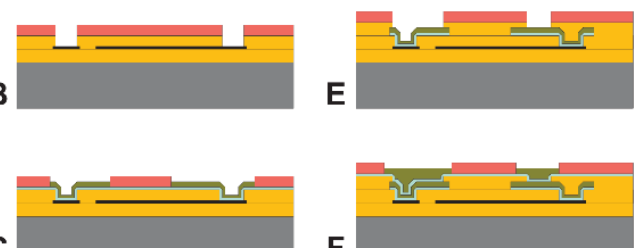

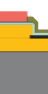

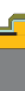

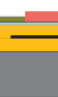
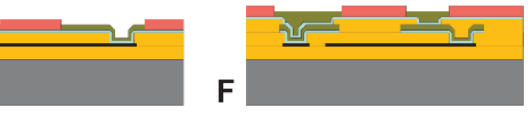

F

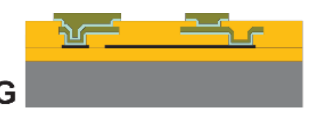

H

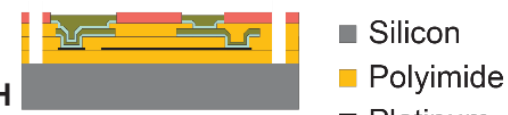

- Platinum

- Photoresist

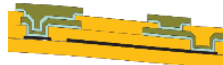

I

$\square \mathrm{Pt} / \mathrm{Au}$ Seed

- Plated Au

\section{Figure S1. Fabrication of the highly flexible oCl substrates.}

(A) Spin coating of first PI layer on Si handle wafer and lift-off patterning of Pt metal lines forming the temperature-sensing element in the first metallization layer. (B) Spin coating of second PI layer, RIE patterning to open vias using photoresist mask. (C) Deposition of metal seed layer and patterned $\mathrm{Au}$ electroplating of the second metallization using a second photoresist mask. (D) Resist strip and seed layer etch. (E) Spin coating of the third PI layer and via opening using a third photoresist mask and RIE. (F) Metal seed layer deposition and patterned Au electroplating of the third metallization. (G) Resist strip and seed layer etch. (H) oCl probe patterning using a fourth photoresist mask and RIE. (I) Photoresist strip and peeling the patterned optical probes off the handle wafer. 


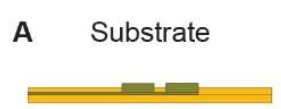

(i)

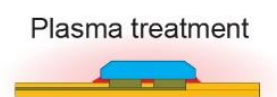

(v)

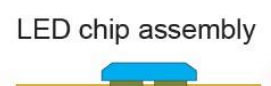

(ii)

Encapsulation

(vi)
Plasma treatment

(iii)

Plasma treatment

(vii)

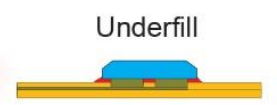

(iv)

Encapsulation

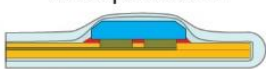

(viii)
- Polyimide
Gold
- LED substrate
- EpoTek
Cytop / Silicone
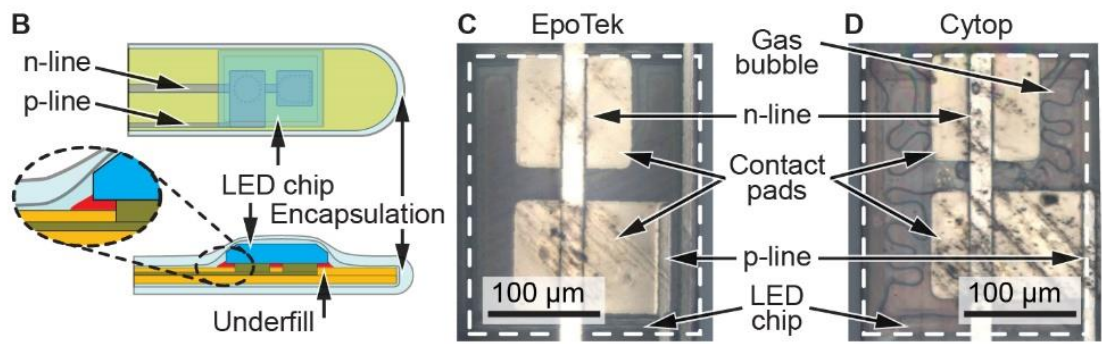

Figure S2. Assembly and passivation of oCls.

(A) Individual LED assembly on the (i) flexible PI substrate using (ii) flip-chip bonding and (iii) surface cleaning and adhesion promotion using argon plasma treatment. (iv) LED underfill with EPO-TEK $301-$ 2. (v) Argon plasma treatment for adhesion promotion and surface cleaning. (vi) Inner Cytop ${ }^{\mathrm{TM}}$ encapsulation. (vii) Oxygen plasma treatment for adhesion promotion and surface cleaning. (viii) Outer silicone encapsulation. (B) Schematic of an LED on a PI substrate illustrating the underfill and exposed materials. (C) Perfectly underfilled LED by using solvent-free EPO-TEK ${ }^{\circ}$ before applying the Cytop $^{\mathrm{TM}}$ - and silicone encapsulation layers. (D) Underfill grade of $77 \%$ by applying Cytop ${ }^{\mathrm{TM}}$ showing gas bubbles. 


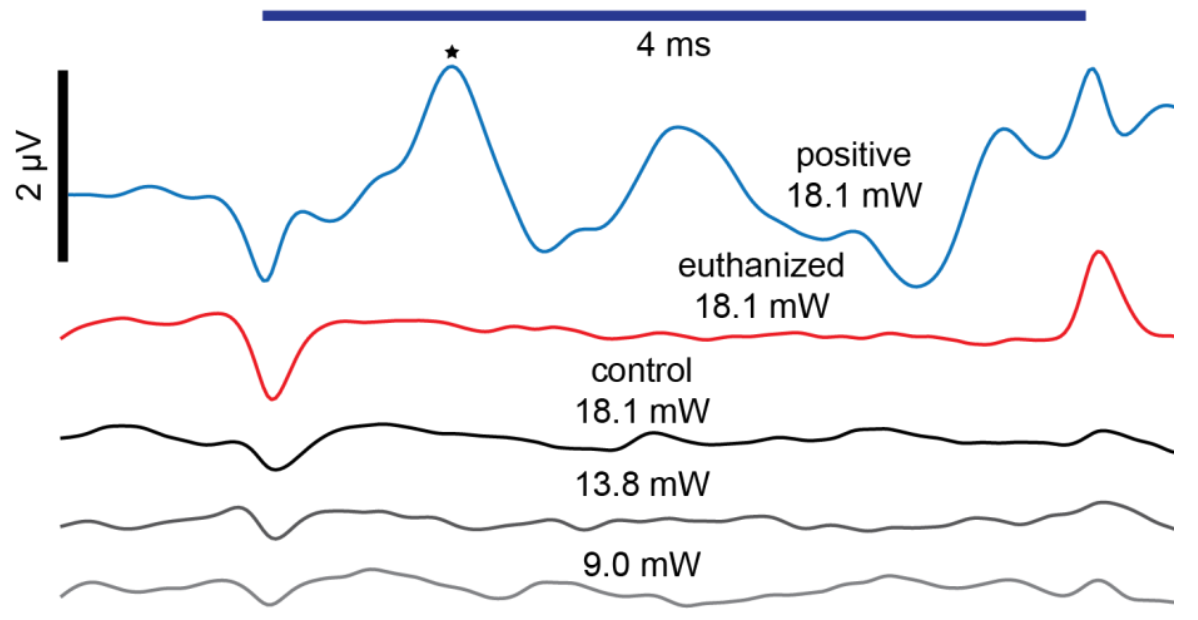

Figure S3. oCls do not evoke potentials in littermate control rats lacking ChR2 or in dead ChR2positive rats.

Upper two traces show stimulus-locked average response to intracochlear LED-based oCl stimulation in a ChR2-positive rat before (blue: clear oABR, p1 labeled by an asterisk, $18.1 \mathrm{~mW}, 10 \mathrm{~Hz}$ stimulus rate, 4 ms stimulus duration) and after euthanizing (red, no oABR, only artifacts) for reference. Lower three traces were obtained from a ChR2-negative littermate rat at different light intensities (9.0 to $18.1 \mathrm{~mW}, 10 \mathrm{~Hz}$ stimulation rate, $4 \mathrm{~ms}$ stimulation duration). The oCl was placed in the middle cochlear turn via a cochleostomy in all cases. Stimulus is displayed as blue bar. 

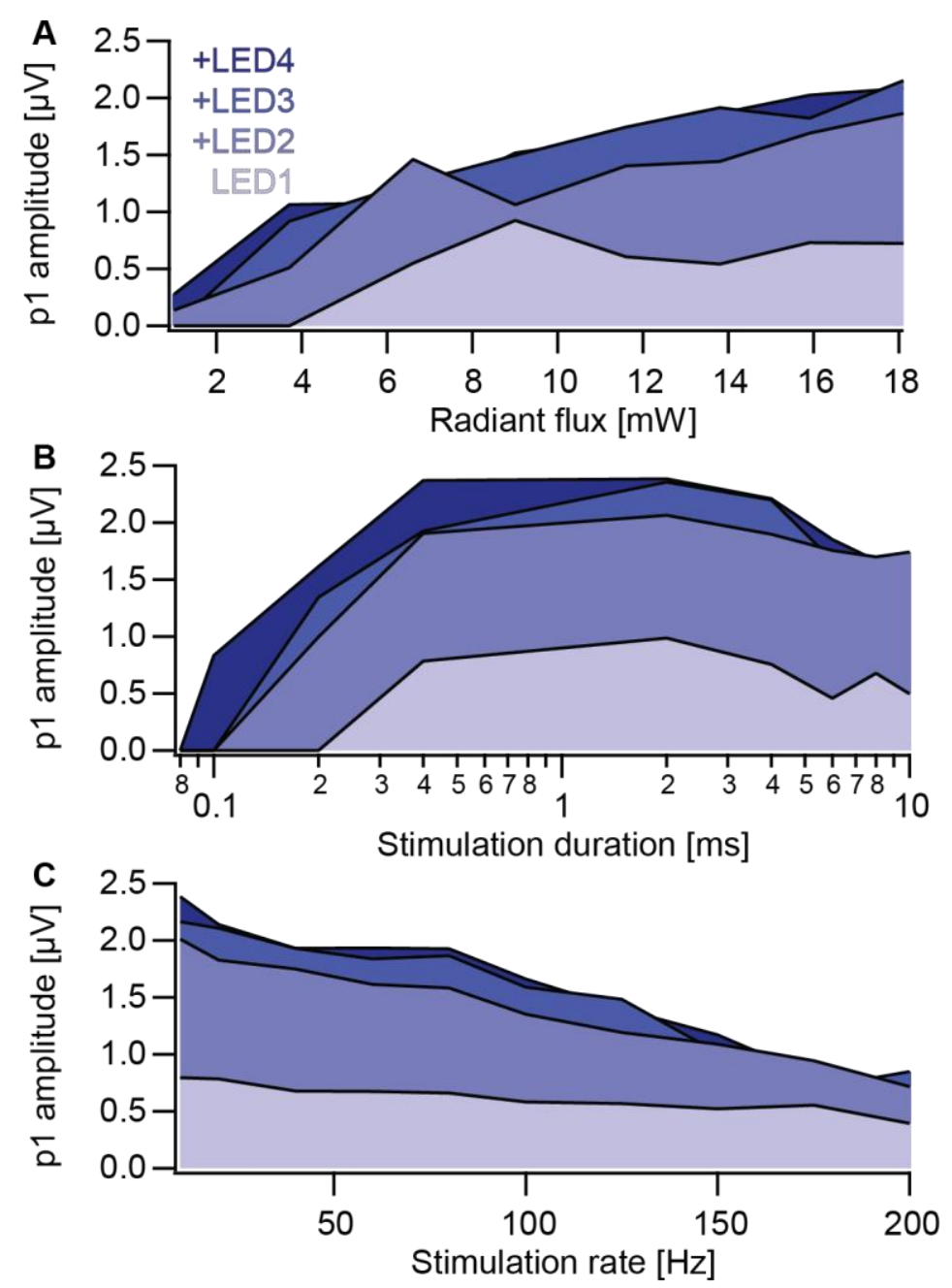

Figure S4. Additivity of LEDs in eliciting OABRs.

The $\mathrm{oCl}$ was placed on the middle cochlear turn via cochleostomy. oABR amplitude of $\mathrm{p} 1$ recorded from one rat with subsequent addition of up to four LEDs (light to dark blue) as a function of radiant flux (A), stimulus duration (B) and stimulation rate (C). LED 1 was inserted for about $3 \mathrm{~mm}$, LED 4 was at the bony edge of the cochleostomy. 


\section{Discussion}

\subsection{Temporal fidelity}

First demonstration of optogenetic stimulation of the auditory nerve in rodents was demonstrated by (Hernandez et al., 2014) in ChR2-transgenic mice under the broad neural promoter Thy1.2. Using transgenic animals was sufficient for first basic questions. Hernandez et al. could show activation of the auditory pathway with blue light. Further, use of a viral vector injected in mouse embryos on day 11.5 with AAV2/6-hSyn carrying the ChR2 mutant CatCh was reported successfully. However, activity broke down at simulation rates higher than $70 \mathrm{~Hz}$, well below the temporal fidelity needed for hearing restoration (Hernandez et al., 2014). The auditory nerve employs spontaneous firing rates of up to $120 \mathrm{~Hz}$ (Liberman, 1978; Schmiedt, 1989) and sustained firing rates of several hundreds of $\mathrm{Hz}$ in mammals (Taberner and Liberman, 2005). For assumptions in humans computational models might close the gap from animal models to humans as physiological studies of the auditory nerve are only available for animals due to the invasive nature of those experiments (Verhulst et al., 2018). Recently, Wrobel et al. 2018 used a direct modiolar AAV-CatCh injection in adult gerbils achieving population responses up to $200 \mathrm{~Hz}$ of stimulation. The difference with the previous study likely indicates more specific activation of the auditory pathway, as indeed, the amplitudes of the optical evoked population responses were comparable to acoustic auditory brainstem responses (Wrobel et al., 2018) while they were 1-3 orders greater in the previous study (Hernandez et al., 2014). Juxtacellular recordings from individual auditory nerve fibers showed well-synchronized responses to light pulses up to approximately $125 \mathrm{~Hz}$ with some fibers following up to $500 \mathrm{~Hz}$ with decreased vector strength. While these results indicate that CatCh-mediated optogenetic activation of SGNs might enable near physiological firing rates, improving temporal fidelity remained an important objective.

Therefore, in chapter 2 we biophysically characterized one of the most promising candidates for translation to humans: Chronos is among the fastest ChRs described to date (Klapoetke et al., 2014). Chronos has been used successfully in the cochlear nucleus (Hight et al., 2015), inferior colliculus (Guo et al., 2015) and the cochlea (Duarte et al., 2018). In our study (Keppeler et al., 2018) we found deactivation time constant of $3 \mathrm{~ms}$ (chapter 2 ) compared to ChR2 ( $\left.\tau_{\text {deact }}=9.4 \mathrm{~ms}\right)$ and CatCh ( $\tau_{\text {deact }} \sim 16 \mathrm{~ms}$ ) at room temperature. At body temperature, the deactivation time constant for Chronos even drops below one millisecond. The ultrafast ion-channel showed sizable oABRs on population level of up to $1000 \mathrm{~Hz}$ of stimulation and firing of single SGNs in response to trains of light pulses at hundreds of $\mathrm{Hz}$ with sub-millisecond temporal precision. These findings promise the possibility of physiological spiking of the optogenetically-driven auditory nerve. 


\subsection{Optogenetic manipulation of spiral ganglion neurons}

ChRs enabling neurons to spike upon light are key to enable optical cochlear stimulation. High transduction efficiency of SGNs within the Rosenthal's canal and efficient trafficking of ChRs in the cell membrane for high light sensitivity and low proteostatic stress are important factors to consider for functional optogenetic hearing restoration. First, the neuronal cells in the cochlea need to be targeted. Most commonly, delivery of genes is achieved via virus-mediated gene transfer. This method harbors the benefit of high flexibility with the use and characterization of new ChR variants (Klapoetke et al., 2014; Mager et al., 2018; Mardinly et al., 2018). Various viruses have been tested for suitability as viral vectors, however, only few are considered safe for application in humans (Kantor et al., 2014).

Usage of viral vectors has the theoretical risks of cell toxicity, their malignant transformation and immune responses. Non-viral vectors have been used to transduce the cochleae with plasmids through electroporation (Pinyon et al., 2014) or attached to nanoparticles (Praetorius et al., 2007) though cell targeting can be limited to directly neighboring cells (Pinyon et al., 2014) and poor transduction efficiency has been reported (Staecker et al., 2001).

Here, we focused on AAV-mediated gene transfer which has been most commonly used and AAV has emerged as one of the safest vectors to date (Lentz et al., 2012). Most critical for AAV-mediated gene transfer is the selection of the appropriate serotype and viral titer while age at injection appears to have less influence on expression levels (Yoshimura et al., 2018). Further, the viral titer correlates directly with general safety (Ramachandran et al., 2017).

While cochlear optogenetics previously embarked on AAV2/6 (Hernandez et al., 2014; Mager et al., 2018) our recent study presented in chapter 2 of this thesis used a novel AAV serotype (Deverman et al., 2016) with improved efficiency of neural transduction. Moreover, after initially employing transuterine AAV-injections we finally focused on postnatal injections that proved efficient and present less harm to the animals. We kept the human synapsin promoter, successfully used before for SGNspecific transduction in the cochlea (Hernandez et al., 2014; Mager et al., 2018). Using postnatal injections of AAV-PHP.B substantial SGN transduction across all cochlear turns was revealed by immunohistochemistry, which is an important advance over the primarily basocochlear expression found with transuterine AAV2/6-injection. While high transduction rates were also found with AAVPHP.B carrying the original Chronos construct, optogenetic stimulation was not efficient owing to the poor membranous expression of Chronos. Only $47 \%$ of Chronos animals showed auditory population responses, while $95 \%$ of injected animals showed reliable peaks for Chronos-ES/TS also using the AAVPHP.B vehicle. Cell membrane targeting is essential for functional expression of Chronos which was achieved appending sequences for improved exit from the endoplasmic reticulum (ES) (Ma et al., 2001; 
Stockklausner et al., 2001) and trafficking to the plasma membrane (TS) (Hofherr, 2005). Improved targeting also comes with the advantage of less proteostasis.

In research, ChRs are fused to a florescent reporter to visualize expression effectively. For translation to humans it might be necessary to remove the reporter due to immune responses caused by the fluorescent protein (Stripecke et al., 1999) which, however, so far has not been reported for immunologically privileged organs like the eye (Dalkara et al., 2013).

\subsection{Light-tissue interaction}

When light is applied to tissue, photons interact with organic material in diverse manners, which can be mainly reduced to scattering and absorption. While scattering mostly alters the propagation path spreading the photons over a large area, absorption leads to energy conversion from light to vibrational energy within the absorbing molecules. To reduce scattering in tissue in the most effective way one could match the refractive index as proposed for in vivo imaging (Boothe et al., 2017), however, this technique would be limited to acute, laboratory experiments and not be feasible for translation. More realistically one could make use of red-shifted ChRs. Naturally, longer wavelengths harbor the benefit of increased penetration depth and more localized targeting of SGNs due to reduced scattering (Jacques, 2013). Narrower spread of excitation would ultimately allow a higher number of stimulation channels. Further, blue-light stimulation harbors a higher risk of photo-toxic damage to cells and tissue (Stockley et al., 2017). In principle, all wavelength of light can be harmful to cells in vitro depending on the dose (Frigault et al., 2009). However, the shorter more energetic wavelengths below $500 \mathrm{~nm}$ are particularly damaging (Wäldchen et al., 2015), thus, stimulation with red-shifted wavelength can reduce potential cellular harm (Mager et al., 2018; Magidson and Khodjakov, 2013). For the commonly used $470 \mathrm{~nm}$ blue light stimulation, a temperature increase of up to $1 \mathrm{~K}$ was reported depending on stimulation protocol (Gysbrechts et al., 2016), not considering additional heat evolving with active stimulators (e.g. $2 \mathrm{~K}$ directly at LED) as described in chapter 4 . Temperature shifts not reaching the threshold for tissue damage might still influence cellular physiology (Moser et al., 1993) and animal behavior (Long and Fee, 2008). Thermal effects towards the SGNs will likely be attenuated due to the circulating perilymph within the scala tympani along with the decay in distance, which is in the range of a few hundred micrometers in mice to several hundred in non-human primates.

\subsection{Advances in oCl development}

Development of optical cochlear implants heavily depends on technical advances to overcome size limitations within the bony boundary of the cochlea. Therefore, we employed phase-contrast X-ray tomography to reveal requirements given by cochlear morphology of commonly used animal models. This detailed study was necessary as a systematic reference was missing. The few studies available 
used various methods from micro-dissection, microscopic analysis to phase-contrast X-ray tomography, which makes direct comparison difficult. Further, the majority of publications focus on cochlear mechanics or eCls and lack volumetric descriptions of the Rosenthal's canal or SGN count which is particularly interesting for optogenetics as the auditory nerve needs to be genetically manipulated, thus requiring injection of a viral vector.

For a light emitting device, an active and a passive design are discussed (Moser, 2015). Active optical stimulators make use of LEDs (Schwaerzle et al., 2016b), $\mu$ LEDs (Goßler et al., 2014; Klein et al., 2018) or OLEDs (Steude et al., 2016). Fitting the micro-scaled scala tympani with its several turns presents a major technological challenge as it requires a highly flexible oCl design and miniaturized light emitters. Further, placing the optoelectronics of active oCls within the cochlea necessitates robust passivation of the probe so that the circuit paths are well isolated from the highly conductive perilymph without the encapsulation absorbing or scattering the emitted light. Passive optical emitters consist of an waveguide array (Schwaerzle et al., 2016a) directing light from remote light sources. Typically verticalcavity surface-emitting lasers (VCSELS) (Tien-Chang Lu et al., 2009) or laser diodes could be used as a light sources. The biggest advantage of a waveguide based oCl would be that all active electronic parts would stay outside the spatially constrained cochlea, thus enabling robust sealed housing e.g. in a titanium case. Further, the intracochlear temperature increase discussed in chapter 4.3 can be largely neglected. A drawback for passive designs is the loss of light occurring during coupling and outcoupling into/from the waveguide. Optical beam-steering could improve the spread of light at the outcoupling spot regardless of the two design modalities: Microlenses (Huang et al., 2017) or microprisms (Reardon et al., 2009). However, the rigid lenses or prisms can negatively impact flexibility of the probe thus impeding smooth implantation into the scala tympani.

Design of $\mu$ LED implant prototypes based on morphological parameters enabled insertion in mouse cochleae for first proof-of-principle experiments. The probe was oriented optimal, with the emitters facing the Rosenthal's canal and an insertion depth of $4.6 \mathrm{~mm}$ corresponding to $71 \%$ of scala tympani. These findings exceed all previously reported implantation depth for mice (Irving et al., 2013; Mistry et al., 2014).

For functional studies we moved on to the larger rat cochlea with oCls carrying larger and powerefficient LEDs for higher light output (chapter 4). The LED arrays could be implanted into the scala tympani, with then usually five intracochlear LEDs, each of which was capable of eliciting oABRs in ann additive manner. Subsequent re-insertion of the optical probe into cochlea demonstrated sufficient mechanical stability. However, artifacts occurred at stimulation onset and offset and flanked or even occluded the optical ABR. Hence, encapsulation and passivation need to be improved to exclude current leakage and interference with electrophysiological recordings. 
Measurements of cochlear morphology helped to develop first chronic oCl experiments and evluate cochlear light spread by computer simulations (Wrobel et al., 2018). Fiber-based single channel implants could be established in a deafened gerbil model. As the light spread of the optical fiber within the cochlea could not be determined, a 3D model was used for Monte Carlo ray tracing to simulate the irradiance at stimulation site. Based on the maximum light intensity used in experiments ( $30 \mathrm{~mW})$, the estimated maximal irradiance at the SGN somata was well within the safe range of optogenetic in vivo applications (Cardin et al., 2010; Han, 2012).

For the first time, we demonstrated an acoustic perception in behavioral experiments upon optical stimulation in the cochlea: In avoidance behavior experiments gerbils responded to sound and light alike indicating an acoustic percept in both cases.

In parallel to improvements specific for optical stimulators, oCls might also profit from ongoing attempts to further advance conventional eCls: One approach focuses on decreasing the distance from stimulator to spiral ganglion within the volume of the scala tympani: a modiolus-hugging probe made of a shape memory polymer could be pre-shaped prior to implantation with a given curvature (Ajieren et al., 2016). The array is straight and relatively stiff prior to insertion which enables directing it towards implantation site. Upon insertion the probe warms up to body temperature and reconstitutes to its coiled shape. Thereby, the memory-shape polymers promise a straightforward insertion and atraumatic modiolus-hugging in $\mathrm{oCl}$ decreasing the distance of Rosenthal's canal.

Also, application of hydrogel particles to the rear side of the $\mathrm{Cl}$ was proposed: Located in the tympanic space of the cochlea, polymer components enlarge by absorbing liquid of the perilymph, thus, passively directing the probe further proximal (Stieghorst et al., 2016).

\subsection{Current and future work}

The key question remains: Is optical stimulation of the cochlea superior to electrical stimulation? How spatially confined is optical stimulation and how much better is the frequency resolution that can be achieved compared to that of eCl? This question cannot be addressed on ABR level, but requires analysis of higher stages along the auditory pathway. Commonly multichannel electrode recordings in the inferior colliculus (IC) or in the auditory cortex are used to indirectly investigate spatial spread of excitation within the cochlea. A preliminary assessment about optical spatial spread of stimulation was provided by (Hernandez et al., 2014) in mice: An optical fiber was placed in the basal turn of the cochlea while recording local field potentials with a multichannel tungsten electrode located in the IC. The largest response was detected in the most ventral region of the IC coding for high frequencies as expected from fiber stimulation close to the round window. Spread of excitation for optogenetic stimulation was comparable to acoustic stimulation and significantly narrower compared to electrical stimulation. 


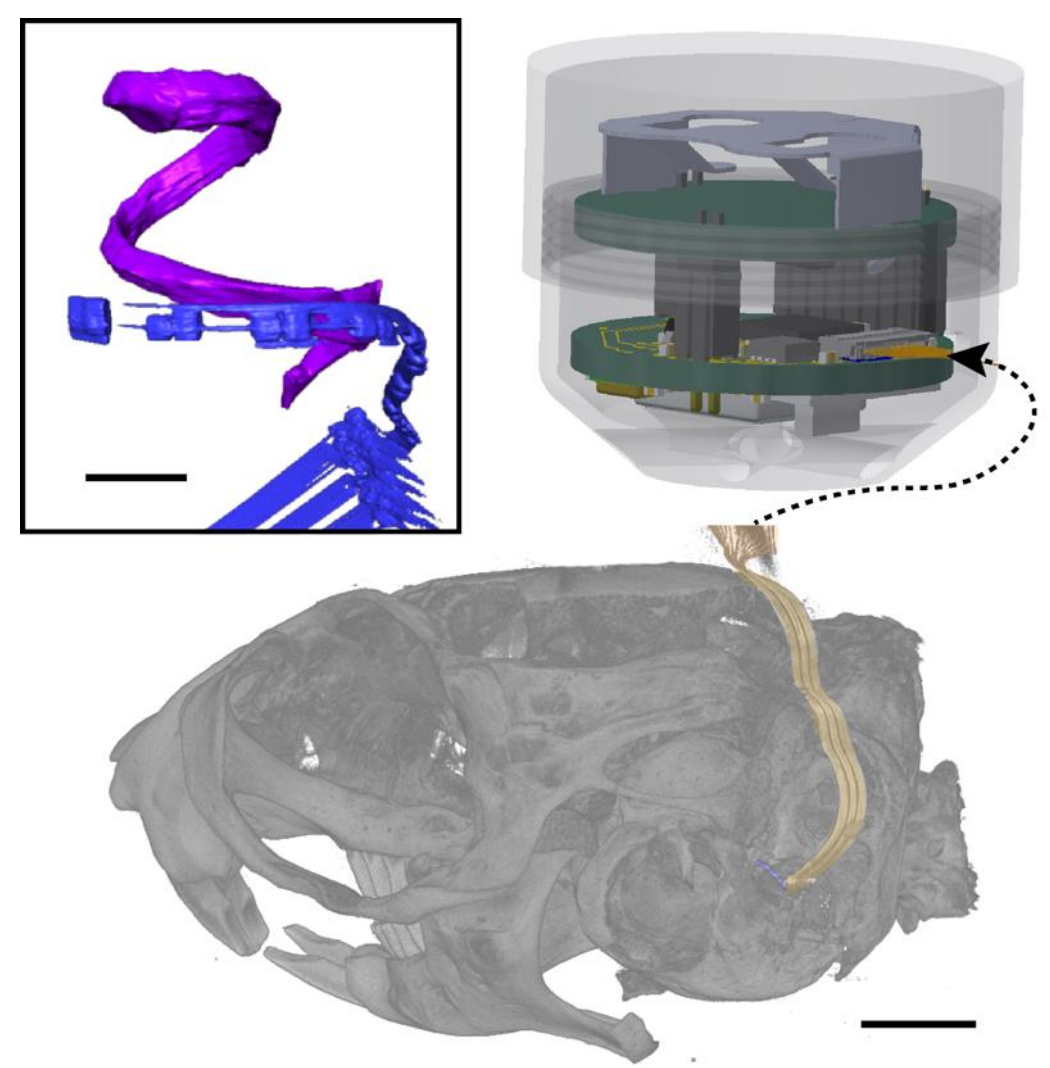

Figure 7. X-ray volume rendering of a chronically implanted gerbil.

Volume rendering of a gerbil chronically implanted with an intracochlear LED probe (blue) and a flexible subcutaneous polyimide cable (orange) reaching the vertex of the gerbil head. The inset shows the well-placed LED array (blue) in the scala tympani facing the Rosenthal's canal (purple). The implantation was atraumatic and did not show damage to the basilar membrane. Scale bar: $0.5 \mathrm{~mm}$. For future experiments, a mobile processor (right top) could be used to enable wireless stimulation. The 3D model of the mobile processor was derived from 2D drawings of Daniel Weihmüller. Scale bar: $3 \mathrm{~mm}$.

However, all current studies so far used single channel optical stimulation. Therefore, chronic implantation with multichannel oCls will be essential to further address remaining questions. Behavioral experiments need to demonstrate the quality of the perceived input. Ongoing efforts focus on improvement of passivation and encapsulation of active oCls to be used for chronic behavioral experiments. In a first proof-of-principle experiment chronic implantation was successful in gerbils (chapter 5, Figure 7) and showed detectable oABRs over the time course of one week (data not shown). However, passivation of the LED probe was not sufficient to prevent stimulation onset and offset artifacts, which were growing over time so that reliable ABR peak detection was not possible after one week. The nature of the artifact has not yet been entirely resolved: During implantation and subsequent bending of the fragile probe, hair-line cracks in the passivation layer could occur. This would allow water to reach the circuit paths through the vapor-permeable silicone layer. Additional passivation, based on chemical vapor deposited polymers like Parylene-C could diminish artifacts (Yang 
et al., 2017). Another possibility would be cold plasma surface treatment for hydrophobization (Waters et al., 2017).

Chronic implants would enable behavior experiments like the discrimination task based on the wellestablished shuttle-box paradigm (Wrobel et al., 2018). Thus, the question on spatial spread and independent channels for oCls could be addressed also based on behavioral experiments. To match the needs for medical application, we have developed a mobile processor to enable optical stimulation in freely-moving animals (chapter 5, Figure 7) in long-term behavior experiments. 


\section{Outlook}

The development of an $\mathrm{oCl}$ is an intricate and multifaceted process. A number of independent technologies must be brought together before clinical translation to the human ear can be realized which essentially includes three steps. (1) Recombinant genetics: SGNs need to be genetically modified to allow light stimulation. Here, most likely adeno-associated viruses will be the vector of choice provided successful demonstration of biosafety, lack of oncogenic potential and long-term stability tests in higher vertebrates. (2) Biological application: the selection of the light-gated ion channel itself, i.e. the $\mathrm{ChR}$ of choice. Defining and evaluating parameters like action spectrum, gating kinetics and ion selectivity of available ChRs are crucial for future success. (3) Engineering technology: Development of appropriate linear arrays of light emitters, considering the limiting microscale dimensions of the intracochlear part, is critical. Here, one possible way is to place an active microscale stimulator within in the cochlea harboring conventional LEDs (Goßler et al., 2014; Klein et al., 2018; Park et al., 2015) or organic LEDs (Steude et al., 2016). Another possibility would be a passive approach using waveguides propagating light from an extracochlear light source (e.g. laser diode, VCSEL) to outcoupling spots placed along the tonotopic axis of the cochlea (Schwaerzle et al., 2016a). Further aspects to consider are number and pitch of the emitters, beam profile, maximum irradiance, available emission wavelengths and built-up of temperature, as well as energy consumption ultimately determining battery life.

The number of independent channels is limited by the spatial spread of the excitation from each emitter and needs to be evaluated. In comparison to electrical stimulation where current readily spreads far from each contact, light can be spatially confined, keeping in mind the aforementioned technical aspects like numerical aperture and emitter size as limiting factors with regards to this. Additionally, knowledge about the amount of light required for auditory perception is scarce to this date necessitating animal behavior experiments to address this question. Furthermore, a coding strategy needs to be developed that will likely differ from the well-established strategies used for electrical stimulation. For example, while electrical stimulation does not allow simultaneously addressing multiple electrodes due to channel cross-talk, multiple emitters can likely be used at the same time for optical stimulation thus enabling stimulation patterns fundamentally different from currently available coding strategies. For validation, optically-evoked electrophysiological data from higher regions of the auditory pathway and psychophysical data are indispensable for developing optical coding strategies. Ultimately, if the $\mathrm{oCl}$ can be developed into a medical device, the optimization of the coding strategy will benefit from feedback of human subjects in clinical trials.

First translation to the clinic might be achieved by using hybrid implants consisting of an $\mathrm{oCl}$ as well as a state-of-the-art eCl. Here, the eCl could act as a backup solution in case of complications with the 
optical stimulation. Furthermore, a combination of electrical and optical stimulation could be discussed with a subthreshold electrical stimulus being presented prior to an optical pulse lowering the respective required threshold.

However, before these can be implemented biocompatibility tests of encapsulation and passivation compounds (e.g. medical grade silicone) are necessary. Long-term stability tests are needed to ensure functionality over decades and to guarantee that metal ions or other substances used for light emitters are well insulated from the patient's body.

All these building blocks are crucial for forthcoming application of oCl in humans. This thesis takes on the project at an early stage of the oCl project thus addressing basic questions for future translation to humans. The main goal of this thesis was to demonstrate feasibility of optogenetic stimulation of the cochlea and lay groundwork for future oCls. Here, the focus laid on genetic modification of SGNs, optimization and characterization of a promising new opsin, the study of morphological features enabling cochlear implantation in common animal models in auditory neuroscience and the demonstration of feasibility of first multi-channel oCls in rats. Beyond the auditory field, conclusions can be related to the field of optogenetics and sensory restoration.

\section{Summary}

This thesis introduces the ultra-fast channelrhodopsin Chronos to the auditory system matching temporal fidelity needed for auditory coding. We provide a comprehensive analysis of the cochlear morphology of animal models commonly used in auditory neuroscience laboratories. Further, we demonstrated, for the first time to my knowledge, first functional results with multichannel optical cochlear implants in rat cochleae. These preclinical studies achieved major steps towards developing the optical cochlear implant for clinical translation. However, many more milestones need to be addressed before the optical cochlear implant can be developed into a medical device for improved hearing restoration. 


\section{Bibliography}

Adamantidis, A.R., Zhang, F., Aravanis, A.M., Deisseroth, K., and Lecea, L. de (2007). Neural substrates of awakening probed with optogenetic control of hypocretin neurons. Nature 450, 420-424.

Ajieren, H., Reit, R., Lee, R., Pham, T., Shao, D., Lee, K., and Voit, W. (2016). Robotic Insertion Aid for Self-Coiling Cochlear Implants. MRS Adv. 1, 51-56.

Bamann, C., Kirsch, T., Nagel, G., and Bamberg, E. (2008). Spectral characteristics of the photocycle of channelrhodopsin-2 and its implication for channel function. J. Mol. Biol. 375, 686-694.

Boothe, T., Hilbert, L., Heide, M., Berninger, L., Huttner, W.B., Zaburdaev, V., Vastenhouw, N.L., Myers, E.W., Drechsel, D.N., and Rink, J.C. (2017). A tunable refractive index matching medium for live imaging cells, tissues and model organisms. ELife 6, e27240.

Boyden, E.S., Zhang, F., Bamberg, E., Nagel, G., and Deisseroth, K. (2005). Millisecond-timescale, genetically targeted optical control of neural activity. Nat. Neurosci. 8, 1263-1268.

Cardin, J.A., Carlén, M., Meletis, K., Knoblich, U., Zhang, F., Deisseroth, K., Tsai, L.-H., and Moore, C.I. (2010). Targeted optogenetic stimulation and recording of neurons in vivo using cell-type-specific expression of Channelrhodopsin-2. Nat. Protoc. 5, 247-254.

Cho, I., Baac, H.W., and Yoon, E. (2010). A 16-site neural probe integrated with a waveguide for optical stimulation. In 2010 IEEE 23rd International Conference on Micro Electro Mechanical Systems (MEMS), pp. 995-998.

Cohen, L.T., Saunders, E., Knight, M.R., and Cowan, R.S.C. (2006). Psychophysical measures in patients fitted with Contour ${ }^{\mathrm{TM}}$ and straight Nucleus electrode arrays. Hear. Res. 212, 160-175.

Dalkara, D., Byrne, L.C., Klimczak, R.R., Visel, M., Yin, L., Merigan, W.H., Flannery, J.G., and Schaffer, D.V. (2013). In Vivo-Directed Evolution of a New Adeno-Associated Virus for Therapeutic Outer Retinal Gene Delivery from the Vitreous. Sci. Transl. Med. 5, 189ra76-189ra76.

Dalkara, D., Goureau, O., Marazova, K., and Sahel, J.-A. (2016). Let There Be Light: Gene and Cell Therapy for Blindness. Hum. Gene Ther. 27, 134-147.

Deisseroth, K. (2011). Optogenetics. Nat. Methods 8, 26-29.

Deverman, B.E., Pravdo, P.L., Simpson, B.P., Kumar, S.R., Chan, K.Y., Banerjee, A., Wu, W.-L., Yang, B., Huber, N., Pasca, S.P., et al. (2016). Cre-dependent selection yields AAV variants for widespread gene transfer to the adult brain. Nat. Biotechnol. 34, 204-209.

Dombrowski, T., Rankovic, V., and Moser, T. (2018). Toward the Optical Cochlear Implant. 15.

Duarte, M.J., Kanumuri, V.V., Landegger, L.D., Tarabichi, O., Sinha, S., Meng, X., Hight, A.E., Kozin, E.D., Stankovic, K.M., Brown, M.C., et al. (2018). Ancestral Adeno-Associated Virus Vector Delivery of Opsins to Spiral Ganglion Neurons: Implications for Optogenetic Cochlear Implants. Mol. Ther. 26, 1931-1939.

Ernst, O.P., Sánchez Murcia, P.A., Daldrop, P., Tsunoda, S.P., Kateriya, S., and Hegemann, P. (2008). Photoactivation of channelrhodopsin. J. Biol. Chem. 283, 1637-1643. 
Fan-Gang Zeng, Rebscher, S., Harrison, W., Xiaoan Sun, and Haihong Feng (2008). Cochlear Implants: System Design, Integration, and Evaluation. IEEE Rev. Biomed. Eng. 1, 115-142.

Fishman, K.E., Shannon, R.V., and Slattery, W.H. (1997). Speech recognition as a function of the number of electrodes used in the SPEAK cochlear implant speech processor. J. Speech Lang. Hear. Res. JSLHR $40,1201-1215$.

Friesen, L.M., Shannon, R.V., Baskent, D., and Wang, X. (2001). Speech recognition in noise as a function of the number of spectral channels: Comparison of acoustic hearing and cochlear implants. J. Acoust. Soc. Am. 110, 1150-1163.

Frigault, M.M., Lacoste, J., Swift, J.L., and Brown, C.M. (2009). Live-cell microscopy - tips and tools. J. Cell Sci. 122, 753-767.

Garnham, C., O'Driscoll, M., Ramsden And, R., and Saeed, S. (2002). Speech understanding in noise with a Med-EI COMBI 40+ cochlear implant using reduced channel sets. Ear Hear. 23, 540-552.

Goßler, C., Bierbrauer, C., Moser, R., Kunzer, M., Holc, K., Pletschen, W., Köhler, K., Wagner, J., Schwaerzle, M., Ruther, P., et al. (2014). GaN-based micro-LED arrays on flexible substrates for optical cochlear implants. J. Phys. Appl. Phys. 47, 205401.

Gunaydin, L.A., Yizhar, O., Berndt, A., Sohal, V.S., Deisseroth, K., and Hegemann, P. (2010). Ultrafast optogenetic control. Nat. Neurosci. 13, 387-392.

Guo, W., Hight, A.E., Chen, J.X., Klapoetke, N.C., Hancock, K.E., Shinn-Cunningham, B.G., Boyden, E.S., Lee, D.J., and Polley, D.B. (2015). Hearing the light: neural and perceptual encoding of optogenetic stimulation in the central auditory pathway. Sci. Rep. 5.

Gysbrechts, B., Wang, L., Trong, N.N.D., Cabral, H., Navratilova, Z., Battaglia, F., Saeys, W., and Bartic, C. (2016). Light distribution and thermal effects in the rat brain under optogenetic stimulation. J. Biophotonics 9, 576-585.

Han, X. (2012). In Vivo Application of Optogenetics for Neural Circuit Analysis. ACS Chem. Neurosci. 3, 577-584.

Hernandez, V.H., Gehrt, A., Reuter, K., Jing, Z., Jeschke, M., Mendoza Schulz, A., Hoch, G., Bartels, M., Vogt, G., Garnham, C.W., et al. (2014). Optogenetic stimulation of the auditory pathway. J. Clin. Invest. $124,1114-1129$.

Hight, A.E., Kozin, E.D., Darrow, K., Lehmann, A., Boyden, E., Brown, M.C., and Lee, D.J. (2015). Superior temporal resolution of Chronos versus channelrhodopsin-2 in an optogenetic model of the auditory brainstem implant. Hear. Res. 322, 235-241.

Hofherr, A. (2005). Selective Golgi export of Kir2.1 controls the stoichiometry of functional Kir2.x channel heteromers. J. Cell Sci. 118, 1935-1943.

Huang, Y., Zhang, X., Zhang, J., Xie, Y., Hofmann, W., Ning, Y., and Wang, L. (2017). Monolithic Microlens VCSELs With High Beam Quality. IEEE Photonics J. 9, 1-8.

Inoue, K., Ito, S., Kato, Y., Nomura, Y., Shibata, M., Uchihashi, T., Tsunoda, S.P., and Kandori, H. (2016). A natural light-driven inward proton pump. Nat. Commun. 7, 13415.

Irving, S., Trotter, M.I., Fallon, J.B., Millard, R.E., Shepherd, R.K., and Wise, A.K. (2013). Cochlear implantation for chronic electrical stimulation in the mouse. Hear. Res. 306, 37-45. 
Jacques, S.L. (2013). Optical properties of biological tissues: a review. Phys. Med. Biol. 58, R37-R61.

Jeschke, M., and Moser, T. (2015). Considering optogenetic stimulation for cochlear implants. Hear. Res. 322, 224-234.

Kantor, B., Bailey, R.M., Wimberly, K., Kalburgi, S.N., and Gray, S.J. (2014). Methods for Gene Transfer to the Central Nervous System. Adv. Genet. 87, 125-197.

Keppeler, D., Merino, R.M., Lopez de la Morena, D., Bali, B., Huet, A., Gehrt, A., Wrobel, C., Subramanian, S., Dombrowski, Tobias, Wolf, F., et al. (2018). Ultrafast optogenetic stimulation of the auditory pathway by targeting-optimized Chronos. EMBO J.

Kiefer, J., von Ilberg, C., Rupprecht, V., Hubner-Egner, J., and Knecht, R. (2000). Optimized speech understanding with the continuous interleaved sampling speech coding strategy in patients with cochlear implants: effect of variations in stimulation rate and number of channels. Ann. Otol. Rhinol. Laryngol. 109, 1009-1020.

Klapoetke, N.C., Murata, Y., Kim, S.S., Pulver, S.R., Birdsey-Benson, A., Cho, Y.K., Morimoto, T.K., Chuong, A.S., Carpenter, E.J., Tian, Z., et al. (2014). Independent optical excitation of distinct neural populations. Nat. Methods 11, 338-346.

Klein, E., Gossler, C., Paul, O., and Ruther, P. (2018). High-density $\mu$ LED-based Optical Cochlear Implant with Improved Thermomechanical Behavior. Front. Neurosci. 12.

Kleinlogel, S., Feldbauer, K., Dempski, R.E., Fotis, H., Wood, P.G., Bamann, C., and Bamberg, E. (2011). Ultra light-sensitive and fast neuronal activation with the $\mathrm{Ca} 2+-$ permeable channelrhodopsin CatCh. Nat. Neurosci. 14, 513-518.

Kohlberg, G., Spitzer, J.B., Mancuso, D., and Lalwani, A.K. (2014). Does cochlear implantation restore music appreciation? The Laryngoscope 124, 587-588.

Kral, A., Hartmann, R., Mortazavi, D., and Klinke, R. (1998). Spatial resolution of cochlear implants: the electrical field and excitation of auditory afferents. Hear. Res. 121, 11-28.

Kuhne, J., Eisenhauer, K., Ritter, E., Hegemann, P., Gerwert, K., and Bartl, F. (2015). Early Formation of the Ion-Conducting Pore in Channelrhodopsin-2. Angew. Chem. Int. Ed. 54, 4953-4957.

Laubsch, A., Sabathil, M., Baur, J., Peter, M., and Hahn, B. (2010). High-Power and High-Efficiency InGaN-Based Light Emitters. IEEE Trans. Electron Devices 57, 79-87.

Lee, J., Nadol, Jr., J.B., and Eddington, D.K. (2010). Depth of Electrode Insertion and Postoperative Performance in Humans with Cochlear Implants: A Histopathologic Study. Audiol. Neurotol. 15, 323331.

Lentz, T.B., Gray, S.J., and Samulski, R.J. (2012). Viral Vectors for Gene Delivery to the Central Nervous System. Neurobiol. Dis. 48, 179-188.

Liberman, M.C. (1978). Auditory-nerve response from cats raised in a low-noise chamber. J. Acoust. Soc. Am. 63, 442-455.

Lin, J.Y., Knutsen, P.M., Muller, A., Kleinfeld, D., and Tsien, R.Y. (2013). ReaChR: a red-shifted variant of channelrhodopsin enables deep transcranial optogenetic excitation. Nat. Neurosci. 16, 1499-1508. 
Long, M.A., and Fee, M.S. (2008). Using temperature to analyse temporal dynamics in the songbird motor pathway. Nature 456, 189-194.

Ma, D., Zerangue, N., Lin, Y.-F., Collins, A., Yu, M., Jan, Y.N., and Jan, L.Y. (2001). Role of ER Export Signals in Controlling Surface Potassium Channel Numbers. Science 291, 316-319.

Mager, T., Lopez de la Morena, D., Senn, V., Schlotte, J., D’Errico, A., Feldbauer, K., Wrobel, C., Jung, S., Bodensiek, K., Rankovic, V., et al. (2018). High frequency neural spiking and auditory signaling by ultrafast red-shifted optogenetics. Nat. Commun. 9.

Magidson, V., and Khodjakov, A. (2013). Circumventing photodamage in live-cell microscopy. Methods Cell Biol. 114

Mardinly, A.R., Oldenburg, I.A., Pégard, N.C., Sridharan, S., Lyall, E.H., Chesnov, K., Brohawn, S.G., Waller, L., and Adesnik, H. (2018). Precise multimodal optical control of neural ensemble activity. Nat. Neurosci. 21, 881-893.

Mistry, N., Nolan, L.S., Saeed, S.R., Forge, A., and Taylor, R.R. (2014). Cochlear implantation in the mouse via the round window: Effects of array insertion. Hear. Res. 312, 81-90.

Moser, T. (2015). Optogenetic stimulation of the auditory pathway for research and future prosthetics. Curr. Opin. Neurobiol. 34, 29-36.

Moser, E., Mathiesen, I., and Andersen, P. (1993). Association between brain temperature and dentate field potentials in exploring and swimming rats. Science 259, 1324-1326.

Nagel, G., Ollig, D., Fuhrmann, M., Kateriya, S., Musti, A.M., Bamberg, E., and Hegemann, P. (2002). Channelrhodopsin-1: A Light-Gated Proton Channel in Green Algae. Science 296, 2395-2398.

Nagel, G., Szellas, T., Huhn, W., Kateriya, S., Adeishvili, N., Berthold, P., Ollig, D., Hegemann, P., and Bamberg, E. (2003). Channelrhodopsin-2, a directly light-gated cation-selective membrane channel. Proc. Natl. Acad. Sci. U. S. A. 100, 13940-13945.

Neumann-Verhoefen, M.-K., Neumann, K., Bamann, C., Radu, I., Heberle, J., Bamberg, E., and Wachtveitl, J. (2013). Ultrafast Infrared Spectroscopy on Channelrhodopsin-2 Reveals Efficient Energy Transfer from the Retinal Chromophore to the Protein. J. Am. Chem. Soc. 135, 6968-6976.

Park, S.I., Brenner, D.S., Shin, G., Morgan, C.D., Copits, B.A., Chung, H.U., Pullen, M.Y., Noh, K.N., Davidson, S., Oh, S.J., et al. (2015). Soft, stretchable, fully implantable miniaturized optoelectronic systems for wireless optogenetics. Nat. Biotechnol. 33, 1280-1286.

Peng, K.A., Lorenz, M.B., Otto, S.R., Brackmann, D.E., and Wilkinson, E.P. (2018). Cochlear implantation and auditory brainstem implantation in neurofibromatosis type 2 . The Laryngoscope 128, 2163-2169.

Pfingst, B.E., and Xu, L. (2004). Across-Site Variation in Detection Thresholds and Maximum Comfortable Loudness Levels for Cochlear Implants. JARO J. Assoc. Res. Otolaryngol. 5, 11-24.

Pinyon, J.L., Tadros, S.F., Froud, K.E., Wong, A.C.Y., Tompson, I.T., Crawford, E.N., Ko, M., Morris, R., Klugmann, M., and Housley, G.D. (2014). Close-Field Electroporation Gene Delivery Using the Cochlear Implant Electrode Array Enhances the Bionic Ear. Sci. Transl. Med. 6, 233ra54-233ra54.

Praetorius, D. >Mark, Brunner, C., Lehnert, B., Klingmann, C., Schmidt, H., Staecker, H., and Schick, B. (2007). Transsynaptic delivery of nanoparticles to the central auditory nervous system. Acta Otolaryngol. (Stockh.) 127, 486-490. 
Prigge, M., Schneider, F., Tsunoda, S.P., Shilyansky, C., Wietek, J., Deisseroth, K., and Hegemann, P. (2012). Color-tuned channelrhodopsins for multiwavelength optogenetics. J. Biol. Chem. 287, 3180431812.

Purves, D. (2018). Neuroscience (New York: Oxford University Press).

Ramachandran, P.S., Lee, V., Wei, Z., Song, J.Y., Casal, G., Cronin, T., Willett, K., Huckfeldt, R., Morgan, J.I.W., Aleman, T.S., et al. (2017). Evaluation of Dose and Safety of AAV7m8 and AAV8BP2 in the NonHuman Primate Retina. Hum. Gene Ther. 28, 154-167.

Reardon, C., Di Falco, A., Welna, K., and Krauss, T. (2009). Optical beam-steering for wireless sensor networks. In 2009 IEEE LEOS Annual Meeting Conference Proceedings, (Belek-Antalya, Turkey: IEEE), pp. 583-584.

Rebscher, S.J., Hetherington, A., Bonham, B., Wardrop, P., Whinney, D., and Leake, P.A. (2008). Considerations for the design of future cochlear implant electrode arrays: Electrode array stiffness, size and depth of insertion. J. Rehabil. Res. Dev. 45, 731-748.

Richter, C.-P., and Tan, X. (2014). Photons and neurons. Hear. Res. 311, 72-88.

Ritter, E., Stehfest, K., Berndt, A., Hegemann, P., and Bartl, F.J. (2008). Monitoring light-induced structural changes of Channelrhodopsin-2 by UV-visible and Fourier transform infrared spectroscopy. J. Biol. Chem. 283, 35033-35041.

Schmiedt, R.A. (1989). Spontaneous rates, thresholds and tuning of auditory-nerve fibers in the gerbil: Comparisons to cat data. Hear. Res. 42, 23-35.

Schwaerzle, M., Nehlich, J., Schwarz, U.T., Paul, O., and Ruther, P. (2016a). Hybrid polymer waveguide characterization for microoptical tools with integrated laser diode chips for optogenetic applications at $430 \mathrm{~nm}$ and $650 \mathrm{~nm}$. S.J. Madsen, V.X.D. Yang, E.D. Jansen, Q. Luo, S.K. Mohanty, and N.V. Thakor, eds. p. $96902 \mathrm{~K}$.

Schwaerzle, M., Nehlich, J., Ayub, S., Paul, O., Patrick, R., and others (2016b). Led-based optical cochlear implant on highly flexible triple layer polyimide substrates. In 2016 IEEE 29th International Conference on Micro Electro Mechanical Systems (MEMS), (IEEE), pp. 395-398.

Schwartz, M.S., and Wilkinson, E.P. (2017). Auditory brainstem implant program development. The Laryngoscope 127, 1909-1915.

Shannon, R., Fu, Q.-J., and Galvin lii, J. (2004). The number of spectral channels required for speech recognition depends on the difficulty of the listening situation. Acta Otolaryngol. (Stockh.) 124, 50-54.

Shannon, R.V., Zeng, F.-G., Kamath, V., Wygonski, J., and Ekelid, M. (1995). Speech Recognition with Primarily Temporal Cues. Science 270, 303-304.

Shichida, Y., and Yamashita, T. (2003). Diversity of visual pigments from the viewpoint of G protein activation-comparison with other $\mathrm{G}$ protein-coupled receptors. Photochem. Photobiol. Sci. 2, 12371246.

Shu, Y., Tao, Y., Wang, Z., Tang, Y., Li, H., Dai, P., Gao, G., and Chen, Z.-Y. (2016). Identification of AdenoAssociated Viral Vectors That Target Neonatal and Adult Mammalian Inner Ear Cell Subtypes. Hum. Gene Ther. 27, 687-699. 
Smith, Z.M., Delgutte, B., and Oxenham, A.J. (2002). Chimaeric sounds reveal dichotomies in auditory perception. Nature 416, 87-90.

Staecker, H., Li, D., O'Malley, B.W., and Van De Water, T.R. (2001). Gene expression in the mammalian cochlea: a study of multiple vector systems. Acta Otolaryngol. (Stockh.) 121, 157-163.

Steude, A., Witts, E.C., Miles, G.B., and Gather, M.C. (2016). Arrays of microscopic organic LEDs for high-resolution optogenetics. Sci. Adv. 2, e1600061-e1600061.

Stieghorst, J., Tran, B.N., Hadeler, S., Beckmann, D., and Doll, T. (2016). Hydrogel-Based Actuation for Modiolar Hugging Cochlear Implant Electrode Arrays. Procedia Eng. 168, 1529-1532.

Stockklausner, C., Ludwig, J., Ruppersberg, J.P., and Klöcker, N. (2001). A sequence motif responsible for ER export and surface expression of Kir2.0 inward rectifier K+ channels. FEBS Lett. 493, 129-133.

Stockley, J.H., Evans, K., Matthey, M., Volbracht, K., Agathou, S., Mukanowa, J., Burrone, J., and Káradóttir, R.T. (2017). Surpassing light-induced cell damage in vitro with novel cell culture media. Sci. Rep. 7.

Stripecke, R., Villacres, M. del C., Skelton, D.C., Satake, N., Halene, S., and Kohn, D.B. (1999). Immune response to green fluorescent protein: implications for gene therapy. Gene Ther. 6, 1305-1312.

Taberner, A.M., and Liberman, M.C. (2005). Response Properties of Single Auditory Nerve Fibers in the Mouse. J. Neurophysiol. 93, 557-569.

Tien-Chang Lu, Jun-Rong Chen, Shih-Wei Chen, Hao-Chung Kuo, Chien-Cheng Kuo, Cheng-Chung Lee, and Shing-Chung Wang (2009). Development of GaN-Based Vertical-Cavity Surface-Emitting Lasers. IEEE J. Sel. Top. Quantum Electron. 15, 850-860.

Verhulst, S., Altoè, A., and Vasilkov, V. (2018). Computational modeling of the human auditory periphery: Auditory-nerve responses, evoked potentials and hearing loss. Hear. Res. 360, 55-75.

Wäldchen, S., Lehmann, J., Klein, T., van de Linde, S., and Sauer, M. (2015). Light-induced cell damage in live-cell super-resolution microscopy. Sci. Rep. 5.

Waters, L.J., Finch, C.V., Bhuiyan, A.K.M.M.H., Hemming, K., and Mitchell, J.C. (2017). Effect of plasma surface treatment of poly(dimethylsiloxane) on the permeation of pharmaceutical compounds. J. Pharm. Anal. 7, 338-342.

WHO (2018). Deafness and hearing loss. accessed 10 October 2018. <http://www.who.int/newsroom/fact-sheets/detail/deafness-and-hearing-loss>.

Wilson, B.S. (1997). Thirty years of the British Journal of Audiology: Guest editorial: The future of cochlear implants. Br. J. Audiol. 31, 205-225.

Wilson, B.S. (2008). Cochlear implants: Current designs and future possibilities. J. Rehabil. Res. Dev. $45,695-730$.

Witten, I.B., Steinberg, E.E., Lee, S.Y., Davidson, T.J., Zalocusky, K.A., Brodsky, M., Yizhar, O., Cho, S.L., Gong, S., Ramakrishnan, C., et al. (2011). Recombinase-driver rat lines: tools, techniques, and optogenetic application to dopamine-mediated reinforcement. Neuron 72, 721-733. 
Wrobel, C., Dieter, A., Huet, A., Keppeler, D., Duque-Afonso, C.J., Vogl, C., Hoch, G., Jeschke, M., and Moser, T. (2018). Optogenetic stimulation of cochlear neurons activates the auditory pathway and restores auditory-driven behavior in deaf adult gerbils. Sci. Transl. Med. 10, eaao0540.

Yang, Y., Zhang, W., Guo, Z., Zhang, Z., Zhu, H., Yan, R., and Zhou, L. (2017). Stability enhanced, repeatability improved Parylene-C passivated on QCM sensor for aPTT measurement. Biosens. Bioelectron. 98, 41-46.

Yizhar, O., Fenno, L., Zhang, F., Hegemann, P., and Diesseroth, K. (2011a). Microbial Opsins: A Family of Single-Component Tools for Optical Control of Neural Activity. Cold Spring Harb. Protoc. 2011, top102.

Yizhar, O., Fenno, L.E., Davidson, T.J., Mogri, M., and Deisseroth, K. (2011b). Optogenetics in Neural Systems. Neuron 71, 9-34.

Yizhar, O., Fenno, L.E., Prigge, M., Schneider, F., Davidson, T.J., O’Shea, D.J., Sohal, V.S., Goshen, I., Finkelstein, J., Paz, J.T., et al. (2011c). Neocortical excitation/inhibition balance in information processing and social dysfunction. Nature $477,171-178$.

Yoshimura, H., Shibata, S.B., Ranum, P.T., and Smith, R.J.H. (2018). Enhanced viral-mediated cochlear gene delivery in adult mice by combining canal fenestration with round window membrane inoculation. Sci. Rep. 8, 2980.

Zeng, H., and Madisen, L. (2012). Mouse transgenic approaches in optogenetics. Prog. Brain Res. 196, 193-213.

Zhang, F., Prigge, M., Beyrière, F., Tsunoda, S.P., Mattis, J., Yizhar, O., Hegemann, P., and Deisseroth, K. (2008). Red-shifted optogenetic excitation: a tool for fast neural control derived from Volvox carteri. Nat. Neurosci. 11, 631-633.

Zhu, Z., Tang, Q., Zeng, F.-G., Guan, T., and Ye, D. (2012). Cochlear Implant Spatial Selectivity with Monopolar, Bipolar and Tripolar Stimulation. Hear. Res. 283, 45-58.

Zorzos, A.N., Boyden, E.S., and Fonstad, C.G. (2010). Multiwaveguide implantable probe for light delivery to sets of distributed brain targets. Opt. Lett. 35, 4133-4135.

Zwolan, T.A., Kileny, P.R., Ashbaugh, C., and Telian, S.A. (1996). Patient performance with the Cochlear Corporation " $20+2$ " implant: bipolar versus monopolar activation. Am. J. Otol. 17, 717-723. 


\section{Acknowledgements}

First, I would like to thank Prof. Tobias Moser for facilitating this dissertation in his lab and email responses on a minute scale. Working in a large team of motivated scientists with excellent collaborations and broad state-of-the-art methods should not be taken for granted. Contributing to the optical cochlear implant project was and still is an exciting part of my life.

Moreover, I thank Prof. Oliver Schlüter and Dr. Katrin Willig the members of my thesis advisory committee for their great support, encouragement, ideas and useful advice.

I would like to express my gratitude to the optogenetics group for great team efforts and many discussions. I appreciate the persistent assistance and technical expertise given by Gerhard Hoch and Peter Wenig who made that project possible. Sandra Gerke and Christiane Senger-Freitag provided excellent technical assistance. Further, I highly appreciated coffee sessions, valuable input on projects and encouragement (in many forms) of my colleagues Alexander Dieter, Dr. Christian Wrobel, Antoine Huet Ph.D., Dr. Fritz Kobe. I wish to thank Dr. Christian Vogl not only but especially for his expert knowledge on immunohistochemistry.

The members of the Institute for Auditory Neuroscience and InnerEarLab are not only great collegues, but also became friends.

Further, I am grateful to Patricia Räke for the constant support and making impossible things possible. I would like to thank the members of University of Freiburg and University of Freiburg for collaborating on the optical cochlear implant project: Dr. Christian Goßler, Dr. Michael Schwaerzle, Eric Klein, Dr. Patrick Ruther and Prof. Ulrich Schwarz.

I would also like to extend my thanks to the laboratory of Prof. Tim Salditt for their collaboration on Xray tomography: Dr. Mareike Töpperwien and Dr. Martin Krenkel were of great support.

The project would not have been possible without the excellent technical skills of the MPI and UMG workshops, namely Rainer Schürkötter and Carsten Lott.

Additionally, I am thankful to the discussions and contributions of all co-authors. And dear Maria, I thank you for our moments, stress release and understanding.

Finally, I wish to thank my parents, brother and family for always being there for me. 


\section{Abbreviations}

AAV

$A B I$

$A B R$

AD

ANOVA

AVCN

BR

CatCh

CCD

ChETA

ChR1

ChR2

$\mathrm{Cl}$

CNS

CT

DA

DC

DIV

DNA

eCl

EDTA

EGFP

ER

ES

EV

EYFP

FIJI

FP

GC

GFP

GPIB

GSDB

$\mathrm{HC}$
Adeno-associated virus

Auditory brainstem implant

Auditory brainstem response

Analog-to-digital

Analysis of variance

Anteroventral cochlear nucleus

Bacteriorhodopsin

Calcium translocating channelrhodopsin

Charge-coupled device

ChR2-E123T accelerated

Channelrhodopsin-1

Channelrhodopsin-2

Cochlear implant

Central nervous system

Computer tomography

Digital-to-analog

Duty cycle

Days in vitro

Deoxyribonucleic acid

Electrical cochlear implant

Ethylenediaminetetraacetic acid

Enhanced green fluorescent protein

Endoplasmic reticulum

Endoplasmic reticulum sequence

Expanded view

Enhanced yellow fluorescent protein

FIJI is just ImageJ

Fluorescent protein

Genome copies

Green fluorescent protein

General purpose interface bus

Goat serum diluted buffer

Hair cell 
HEK cell

HF

$\mathrm{HI}$

HR

hSyn

IHC

KI

LED

$\mathrm{NI}$

OABR

$\mathrm{oCl}$

$\mathrm{OHC}$

OLED

PBS

PCB

pcDNA

PCR

PFA

PHP

PI

PMMA

PR

RIE

RT

RT

RW

SD

SEM

SGN

SNHL

STED microscopy

TDE

TS

USB

UV
Human embryonic kidney cells

Hydrofluoric acid

Hearing impairment

Halorhodopsin

Human synapsin

Inner hair cell

Potassium iodide

Light-emitting diode

National instruments

Optical auditory brainstem response

Optical cochlear implant

Outer hair cell

Organic light-emitting diode

Phosphate-buffered saline

Printed circuit board

Protamine complementary DNA

Polymerase chain reaction

Paraformaldehyde

Paul H. Patterson

Polyimide

Polymethylmethacrylat

Proteorhodopsin (see BR)

Reactive ion etching

Room temperature

Room temperature

Round window

Standard deviation

Standard error of the mean

Spiral ganglion neuron

Sensory neural hearing loss

Stimulated emission depletion microscopy

2,2-Thiodiethanol

Trafficking sequence

Universal serial bus

Ultraviolet 
VChR1

VCSEL

WHO

YFP

ZIF
Volvox carteri channelrhodopsin-1

Vertical-cavity surface-emitting laser

World health organization

Yellow fluorescent protein

Zero insertion force 


\section{List of figures}

\section{Introduction}

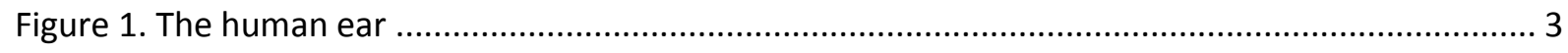

Figure 2. Travelling wave along the uncoiled cochlea.......................................................................... 4

Figure 3. Schematic overview of different cochlear stimulation modalities........................................ 8

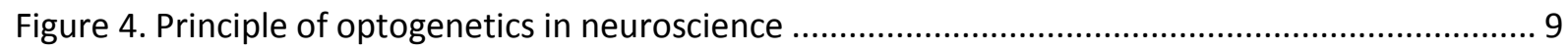

Figure 5. Schematic of basic function of rhodopsins .................................................................... 9

Figure 6. Opsin kinetics and peak absorbation wavelenghth for common optogenetic tool variants . 11

\section{Ultrafast optogenetic stimulation of the auditory pathway by targeting-optimized Chronos}

Figure 1. Chronos mediates fast light-driven currents.

Figure 2. Optimizing membrane expression of Chronos by adding ER-exit and trafficking signals: HEK293T cells 19

Figure 3. Improved membrane expression of optimized Chronos-ES/TS in hippocampal neurons ..... 20

Figure 4. Establishing efficient expression of Chronos in SGNs: use of Chronos-ES/TS, potent AAVPHP.B, and postnatal mode of AAV-injection

Figure EV1. Demonstrating functional expression of Chronos in SGNs following transuterine AAV2/6 injection reported by recordings of oABRs

Figure 5. Characterizing optogenetic stimulation by recordings of oABRs. 26

Figure 6. Characterizing optogenetic stimulation by extracellular recordings from single putative SGNs

Figure EV2. Activity of two exemplary putative SGNs upon light stimulation at increasing repetition rates. 30

Multiscale, multimodal imaging of the native and implanted cochlea

Figure 1. 3D models of rodent and primate cochleae....... 51

Figure 2. Measurements of the scala tympani crucial for cochlear implantation 52

Figure 3. Implantation trials in rodent cochleae 55

Figure 4. Implantation trials in marmoset cochleae 56

Figure S1. Cross-sectional area and perimeter .62

Figure S2. Implantation trials of dip-coated polyimide-based probes in mouse cochleae. 63 
Towards optical cochlear implants: first multichannel optogenetic stimulation of the auditory pathway using microfabricated LED implants

Figure 1. Design of the LED-based multichannel optical cochlear implants. ....................................... 74

Figure 2. Electrical, optical and thermal characterization of an oCl. ................................................ 77

Figure 3. oCl stimulation of the auditory pathway in ChR2 transgenic rats. ...................................... 79

Figure 4. Characterizing oCl stimulation by recordings of oABRs in rats.......................................... 81

Figure S1. Fabrication of the highly flexible oCl substrates. .............................................................. 91

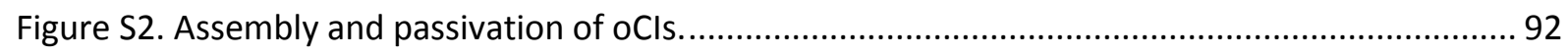

Figure S3. oCls do not evoke potentials in littermate control rats lacking ChR2 or in dead ChR2-positive

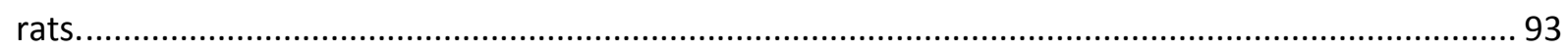

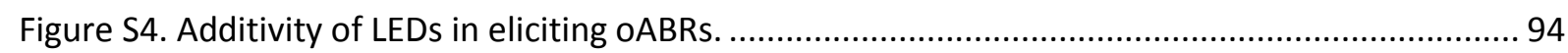

\section{$\underline{\text { Outlook }}$}

Figure 7. X-ray volume rendering of a chronically implanted gerbil. ............................................... 100 


\section{List of tables}

Table 1. Comparison of cochlear measurements across species ..................................................... 53

Table 2. Comparision of cochlear implant insertion depth across species .......................................... 57

Table S1. Comparison of cochlear measurements to literature values ................................................. 64 


\section{Declaration}

I hereby declare that my dissertation entitled "Ultrafast Multichannel Optogenetic Stimulation of the Auditory Pathway for Optical Cochlear Implants" has been written independently and with no other sources and aids than quoted.

Göttingen, January 10, 2019

Daniel Keppeler 


\section{Curriculum vitae}

\section{Daniel Keppeler, M. Sc.}

Springstraße $21 \bullet 37077$ Göttingen, Germany

daniel.keppeler@med.uni-goettingen.de

\section{Research Summary}

The research of my PhD focusses on the development of an innovative optogenetics-based cochlear implant $(\mathrm{oCl})$, combining viral transduction in rodents with electrophysiological and immunohistochemical methods. Employing 3D micro-scale X-ray tomography enabled me to study cochlear morphology and implant position.

\section{Education}

- PhD candidate, Sensory and Motor Neuroscience, University Medical Center Göttingen, Germany, since 2014

Thesis: "Ultrafast Multichannel Optogenetic Stimulation of the Auditory Pathway for Optical Cochlear Implants", supervised by Prof. Tobias Moser

- M. Sc., Molecular Medicine, University Medical Center Göttingen, Germany, 2012-2014

Thesis: "Refining optogenetic stimulation of the cochlea: Chronos, a new fast channelrhodopsin variant and $\mu$ LED cochlea implants as a new approach to restore hearing", supervised by Prof. Tobias Moser

- B. Sc., Trophology, University of Jena, Germany, 2009-2012

Thesis: "Vitamins and Bioactive Compounds in Testa and Kernels of Cashews (Anacardium occidentale) and Peanuts (Arachis hypogaea)", supervised by Prof. Tilman Grune

\section{Stays abroad}

- Internship, assistant of the construction-site supervision of "7 More London Riverside" London, UK for seele GmbH UK Branch, 2009

Tasks: logistics, translations and construction report

- Civilian service, Beannachar Camphill Community in Aberdeen, Scotland, UK, 2008

Tasks: Care for people with special needs in a living community

\section{Awards}

- Junior scientist award 2015 on the 18th Annual Meeting of the German Audiology Society (DGA)

\section{Teaching}

- B.Sc. supervision Ringo Mielke

Thesis: "Das Multiphotonenmikroskop" Setupoptimierung zur in vivo - Funktionsanalyse des auditorischen Cortex einer Maus mit synchron gesteuerter akustischer Reizung"

\section{Experimental experience}

- Cell culture, immunohistochemistry, confocal microscopy

- Microsurgery in rodents (stereotactic injections, inner ear surgery, craniotomy)

- Electrophysiology in anesthetized rodents

- X-ray tomography and 3D image analysis

- Programming using Matlab 
- Laboratory animal handling, management and welfare - Felasa B

- Good Manufacturing Practice

\section{Publications}

Keppeler, D.*, Merino, R.M.*, Lopez de la Morena, D.* , Bali, B., Huet, A.T., Gehrt, A., Wrobel, C., Subramanian, S., Dombrowski, T., Wolf, F., Rankovic, V., Neef, A. and Moser, T. (2018). Ultrafast optogenetic stimulation of the auditory pathway by targeting-optimized Chronos. EMBO Journal. DOI: 10.15252/embj.201899649. (2018). *equal author contributions.

Keppeler, D., Duque-Afonso, C.J., Töpperwien, M., Meyer, A., Moser T. Multiscale, multimodal imaging of the native and implanted cochlea. (in preparation)

Keppeler, D.*, Schwaerzle, M.*, Nehlich, J., Harczos, T., Wrobel, C., Ringwald, P., Raz, R., Hoch, G., Abdellatif, K., Bodensiek, K., Paul, O., Ruther, P. and Moser, T. Towards optical cochlear implants: first multichannel optogenetic stimulation of the auditory pathway using microfabricated LED implants. (under revision) *equal author contributions.

Wrobel, C.*, Dieter, A.*, Huet, A.\#, Keppeler, D.\#, Duque-Afonso, C.J., Vogl, C., Hoch, G., Jeschke, M., and Moser, T. (2018). Optogenetic stimulation of cochlear neurons activates the auditory pathway and restores auditory-driven behavior in deaf adult gerbils. Science Translational Medicine 10, eaao0540. *,"equal author contributions.

Töpperwien, M., Gradl, R., Keppeler, D., Vassholz, M., Meyer, A., Hessler, R., Achterhold, K., Gleich, B., Dierolf, M., Pfeiffer, F., Moser, T., and Salditt, T. (2018). Propagation-based phase-contrast x-ray tomography of cochlea using a compact synchrotron source. Sci Rep 8.

Goßler, C., Bierbrauer, C., Moser, R., Kunzer, M., Holc, K., Pletschen, W., Köhler, K., Wagner, J., Schwaerzle, M., Ruther, P., Paul, O., Neef, J., Keppeler, D., Hoch, G., Moser, T., and Schwarz, U.T. (2014). GaN-based micro-LED arrays on flexible substrates for optical cochlear implants. Journal of Physics D: Applied Physics 47, 205401. 


\section{Conference contributions}

- "First multichannel optical cochlear implants for optogenetic stimulation of the auditory pathway," talk presented at the Conference on Implantable Auditory Prostheses (CIAP), Lake Tahoe, California, US, 16-Jul-2017.

- "Characterizing optogenetic stimulation of the auditory pathway using multichannel optical Cls," talk presented at the 53rd Inner Ear Biology (IEB), Montpellier, FR, 19-Sep-2016.

- "Characterizing cochlear morphology and preliminary insertion studies with multichannel optical Cls," talk and poster presented at the Restoration of Sensory and Motor Function (RSMF), Göttingen, DE, 26-May-2016.

- "Ultrafast optogenetic cochlea stimulation and development of microLED implants for research and clinical application," poster presented at the Conference on Implantable Auditory Prostheses (CIAP), Lake Tahoe, California, US, 07-Dec-2015.

- "In vivo application of optogenetics in the auditory system," invited talk presented at the 95th Annual Meeting of the German Physiological Society (DPG), Magdeburg, DE, 03-May-2015.

- "Optogenetic stimulation of the auditory pathway in research and future prosthetics," invited talk presented at the 8th Student Symposium on Molecular Medicine, Ulm, DE, 04-Apr-2015.

- "Optogenetic stimulation of the auditory pathway," talk presented at the 18th Annual Meeting of the German Audiology Society (DGA), Bochum, DE, 03-Jun-2015.

- "Towards optogenetic stimulation of the auditory pathway: Implementing the fast channelrhodopsin Chronos and multichannel microLED arrays," short talk and poster presented at the 38th Annual MidWinter Meeting of the Association for Research in Otolaryngology (ARO), Baltimore, Maryland, US, 21-Feb-2015.

- "Establishing fast cochlear optogenetics: Chronos-based stimulation of the auditory pathway," poster presented at the Conference on Channelrhodopsin et al. - Optogenetic Tools \& Application, Würzburg, DE, 29-Sep-2014.

- "Zukünftige Innovationen in Hörprothesen - Channelrhodopsin-2 vermittelte optische Stimulation der Cochlea," invited talk presented at the 20. Friedberger Cochlea Implantat- und HörsystemSymposium, Bad Nauheim, DE, 06-May-2014. 